BNL-HET-01/33

\title{
High Transverse Momentum Physics at the Large Hadron Collider
}

\author{
The ATLAS and CMS Collaborations \\ Edited by \\ J.G. Branson ${ }^{\mathrm{a}}$, D. Denegri ${ }^{\mathrm{b}}$, I. Hinchliffe ${ }^{\mathrm{c}}$, F. Gianotti ${ }^{\mathrm{d}}$, F.E. Paige ${ }^{\mathrm{e}}$, and P. Sphicas ${ }^{\mathrm{d}, \mathrm{f}}$ \\ ${ }^{a}$ U. of California San Diego, La Jolla, CA 92093 \\ ${ }^{b}$ DAPNIA, CEA/Saclay, F-91191 Gif-sur-Yvette, France \\ ${ }^{c}$ Lawrence Berkeley National Laboratory, Berkeley, CA 94720 \\ ${ }^{d}$ CERN, CH-1211 Geneva 23, Switzerland \\ ${ }^{e}$ Brookhaven National Laboratory, Upton, NY 11973 \\ ${ }^{f}$ MIT, Cambridge, MA 02139
}

INTRODUCTION AND MOTIVATION

A The Standard Model . . . . . . . . . . . . . . .

B Beyond the Standard Model. . . . . . . . . .

II THE LARGE HADRON COLLIDER

A Machine parameters ............

B Physics Goals . . . . . . . . . . . . .

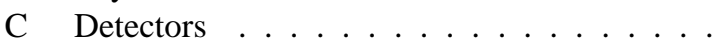

III

HIGGS PHYSICS

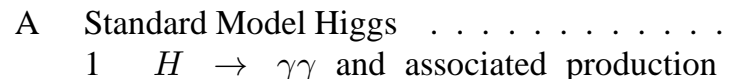
channels . . . . . . . . . .

$2 \quad H \rightarrow b b \ldots \ldots \ldots \ldots \ldots$

$3 \quad H \rightarrow Z Z^{*} \rightarrow 4 \ell \ldots \ldots \ldots \ldots$

$4 \quad H \rightarrow W W^{(*)} \rightarrow \ell^{+} \nu \ell^{-}$. $\ldots \ldots . .9$

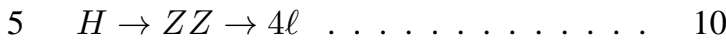

$6 \quad M_{H} \sim 1 \mathrm{TeV}(H \rightarrow \ell \ell \nu \nu, \ell \ell j j, \ell \nu j j)$. 10

$7 \quad$ Measurements of Higgs properties _ . . . 12

$8 \quad$ Summary of Standard Model Higgs . . . 13

B SUSY Higgs . .................. 13

$11 \quad H / A \rightarrow \tau \tau \ldots \ldots \ldots \ldots . \ldots . \ldots 14$

$2 \quad H / A \rightarrow \mu \mu \mid \ldots \ldots \ldots \ldots \ldots$

$3 \quad A \rightarrow \gamma \gamma \ldots \ldots \ldots \ldots \ldots \ldots$

$4 \quad$ Search for Charged Higgs $\ldots \ldots \ldots$

5 Other possible Higgs signatures . . . . . 15

6 Summary of Supersymmetric Higgs . . . 16

IV SUPERSYMMETRY

A $\quad$ SUGRA Measurements . . . . . . . . . . . . . 17

B $\quad$ GMSB Measurements. . . . . . . . . . . . . 21

C AMSB Measurements . . . . . . . . . . . . . 22

D $\quad R$-Parity Violation . . . . . . . . . . . . . 22

E Decay of Higgs to Sparticles . . . . . . . . 22

F SUSY Summary . . . . . . . . . . . . . . 23

\begin{tabular}{|ll}
\hline S & STRONG EWSB DYNAMICS \\
\hline
\end{tabular}

A Strongly interacting $W$ ' . . . . . . . . . . . . 24

B $\quad$ Technicolor ................ . . 25

C Compositeness .............. 25

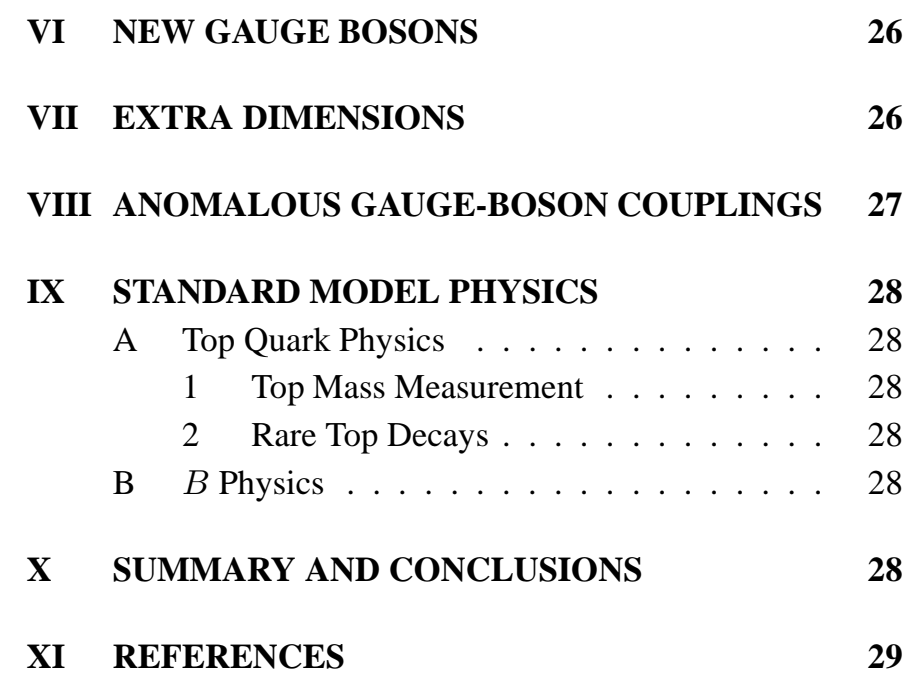

\section{ABSTRACT}

This note summarizes many detailed physics studies done by the ATLAS and CMS Collaborations for the LHC, concentrating on processes involving the production of high mass states. These studies show that the LHC should be able to elucidate the mechanism of electroweak symmetry breaking and to study a variety of other topics related to physics at the $\mathrm{TeV}$ scale. In particular, a Higgs boson with couplings given by the Standard Model is observable in several channels over the full range of allowed masses. Its mass and some of its couplings will be determined. If supersymmetry is relevant to electroweak interactions, it will be discovered and the properties of many supersymmetric particles elucidated. Other new physics, such as the existence of massive gauge bosons and extra dimensions can be searched for extending existing limits by an order of magnitude or more".

\footnotetext{
${ }^{1}$ Note that this document lacks the detector pictures due to file size limiations of the Los Alamos archive. The version with pictures can be found at http://www-theory.lbl.gov/_ ianh/lhc
} 


\section{INTRODUCTION AND MOTIVATION}

This document summarizes the potential of the Large Hadron Collider (LHC) for high mass and high transverse momentum physics and explains why the LHC is expected to provide a crucial next step in our understanding of nature. The results given here are based on publically available work done by many ATLAS and CMS collaborators either as part of the design of the ATLAS [1] and CMS [2] detectors or in subsequent investigations. On the basis of these studies, we believe that the physics potential of the LHC is enormous: among currently approved projects in high energy physics, it uniquely has sufficient energy and luminosity to probe in detail the $\mathrm{TeV}$ energy scale relevant to electroweak symmetry breaking.

\section{A. The Standard Model}

The Standard Model (SM) is a very successful description of the interactions of the components of matter at the smallest scales $\left(\lesssim 10^{-18} \mathrm{~m}\right)$ and highest energies $(\sim 200 \mathrm{GeV})$ accessible to current experiments. It is a quantum field theory that describes the interaction of spin- $\frac{1}{2}$, point-like fermions, whose interactions are mediated by spin- 1 gauge bosons. The existence of the gauge bosons and the form of their interactions are dictated by local gauge invariance, a manifestation of the symmetry group of the theory, which for the $\mathrm{SM}$ is $S U(3) \times S U(2) \times U(1)$.

The fundamental fermions are leptons and quarks; the lefthanded states are doublets under the $S U(2)$ group, while the right-handed states are singlets. There are three generations of fermions, each generation identical except for mass. The origin of this structure, and the breaking of generational symmetry (flavor symmetry) remain a mystery. There are three leptons with electric charge -1 , the electron $(e)$, muon $(\mu)$ and tau lepton $(\tau)$; and three electrically neutral leptons, the neutrinos $\nu_{e}, \nu_{\mu}$ and $\nu_{\tau}$. Similarly there are three quarks with electric charge $+\frac{2}{3}$, up $(u)$, charm $(c)$ and top $(t)$; and three with electric charge $-\frac{1}{3}$, down $(d)$, strange $(s)$ and bottom $(b)$. The quarks are triplets under the $S U(3)$ group and thus carry an additional "charge," referred to as color. There is mixing between the three generations of quarks, which in the SM is parameterized by the Cabibbo-Kobayashi-Maskawa (CKM) [3] matrix but is not explained.

In the SM the $S U(2) \times U(1)$ symmetry group describes the electroweak interactions. This symmetry is spontaneously broken by the existence of a (postulated) Higgs field with a nonzero expectation value, leading to massive vector bosons - the $W^{ \pm}$and $Z$ - which mediate the weak interaction; the photon of electromagnetism remains massless. One physical degree of freedom remains in the Higgs sector, a neutral scalar boson $H^{0}$, which is presently unobserved. The $S U(3)$ group describes the strong interaction (quantum chromodynamics or QCD). The eight vector gluons that mediate this interaction themselves carry color charges and so are self-interacting. This implies that the QCD coupling $\alpha_{S}$ is small for large momentum transfers but large for small momentum transfers, and leads to the confinement of quarks inside color-neutral hadrons. Attempting to free a quark produces a jet of hadrons through quark-antiquark pair production and gluon bremsstrahlung. The smallness of the strong coupling at large mass scales makes it possible to calculate reliably cross sections for the production of massive particles at the LHC.

The basic elements of the Standard Model were proposed in the 1960's and 1970's [4]. Increasing experimental evidence of the correctness of the model accumulated through 1970's and 1980's:

- observation of (approximate) scaling in deep inelastic scattering experiments, showing the existence of point-like scattering centers inside nucleons, later identified with quarks [5];

- observation of the $c$ and $b$ quarks [6];

- observation of neutral weak currents from $Z$ exchange [䜣;

- observation of jet structure and three-jet final states resulting from gluon radiation in $e^{+} e^{-}$and hadron-hadron collisions [8];

- direct observation of the $W$ and $Z$ at the CERN $S p \bar{p} S$ collider [9].

Following these discoveries, ever more precise experiments at LEP and SLC have provided verification of the couplings of quarks and leptons to the gauge bosons at the level of 1loop radiative corrections $\left(\sim \mathcal{O}\left(10^{-3}\right)\right)$. Also, the top quark has been discovered at Fermilab with a very large mass $(\sim$ $175 \mathrm{GeV})[10]$.

With the recent direct observation of the $\nu_{\tau}$ [11], only one particle from the Standard Model has yet to be observed, the Higgs boson. The Higgs is very important because it holds the key to the generation of $W, Z$, quark and lepton masses.

Some of the SM parameters, specifically those of the CKM matrix, are not well determined. In particular, while CP violation is accommodated in the SM through a phase in the CKM quark mixing matrix, it remains poorly understood. CP violation was first observed in $\mathrm{K}$ decays [12]. Recently, direct $\mathrm{CP}$ violation has been seen in $\mathrm{K}$ decays [13], and evidence for $\mathrm{CP}$ violation in $B \rightarrow \psi K_{s}$ has been seen in $B$-factories [14, 15] and in CDF [16]. More precise measurements over the next few years should determine these parameters or demonstrate the SM cannot adequately explain $\mathrm{CP}$ violation.

The minimal SM can only accommodate massless neutrinos and hence no neutrino oscillations. There is evidence for such oscillations from measurements by SuperKamiokande of neutrinos produced in the atmosphere and from a deficit in the flux of electron neutrinos from the sun[17]. While it is easy to extend the SM to include neutrino masses, understanding their small values seems to require qualitatively new physics.

\section{B. Beyond the Standard Model}

The success of the Standard Model [4] of strong (QCD), weak and electromagnetic interactions has drawn increased attention to its limitations. In its simplest version, the model has 19 parameters [18], the three coupling constants of the gauge theory $S U(3) \times S U(2) \times U(1)$, three lepton and six quark masses, 
the mass of the $Z$ boson which sets the scale of weak interactions, the four parameters which describe the rotation from the weak to the mass eigenstates of the charge $-1 / 3$ quarks (CKM matrix). All of these parameters are determined with varying errors. One of the two remaining parameters is the coefficient $\theta$ of a possible $C P$-violating interaction among gluons in QCD; limits on the $C P$ violation in strong interactions imply that it must be very small. The other parameter is associated with the mechanism responsible for the breakdown of the electroweak $S U(2) \times U(1)$ to $U(1)_{\mathrm{em}}$. This can be taken to be the mass of the as yet undiscovered Higgs boson, whose couplings are determined once its mass is given. Additional parameters are needed to accommodate neutrino masses and mixings.

The gauge theory part of the SM has been well tested, but there is little direct evidence either for or against the simple Higgs mechanism for electroweak symmetry breaking. The current experimental lower bound on the Higgs mass is 113.5 GeV[19]. If the Standard Model Higgs sector is correct, then precision measurements at the $Z$ and elsewhere can be used to constrain the Higgs mass via its contribution to the measured quantities from higher order quantum corrections to be less than $212 \mathrm{GeV}$ [20] at $95 \%$ confidence. As the Higgs mass increases, its self couplings and its couplings to the $W$ and $Z$ bosons grow [21]. This feature has a very important consequence. Either the Higgs boson must have a mass less than about $800 \mathrm{GeV}$ or the dynamics of $W W$ and $Z Z$ interactions with center of mass energies of order $1 \mathrm{TeV}$ will reveal new structure. It is this simple argument that sets the energy scale that must be probed to guarantee that an experiment will be able to provide information on the nature of electroweak symmetry breaking.

The presence of a single elementary scalar boson is distasteful to many physicists. If the theory is part of some more fundamental theory, which has some other larger mass scale (such as the scale of grand unification or the Planck scale), there is a serious "fine tuning" or naturalness problem. Radiative corrections to the Higgs boson mass result in a value that is driven to the larger scale unless some delicate cancellation is engineered $\left(m_{0}^{2}-m_{1}^{2} \sim M_{W}^{2}\right.$ where $m_{0}$ and $m_{1}$ are order $10^{15} \mathrm{GeV}$ or larger). There are two ways out of this problem which involve new physics at a scale of order $1 \mathrm{TeV}$. New strong dynamics could enter that provides the scale of $M_{W}$, or new particles could appear so that the larger scale is still possible, but the divergences are canceled on a much smaller scale. It is also possible that there is no higher scale as, for example in models with extra dimensions. In any of the options, Standard Model, new dynamics or cancellations, the energy scale is the same: something must be discovered on the TeV scale.

Supersymmetry is an appealing concept for which there is, at present, no experimental evidence [22]. It offers the only presently known mechanism for incorporating gravity into the quantum theory of particle interactions, and it provides an elegant cancellation mechanism for the divergences provided that at the electroweak scale the theory is supersymmetric. The successes of the Standard Model (such as precision electroweak predictions) are retained, while avoiding any fine tuning of the Higgs mass. Some supersymmetric models allow for the unification of gauge couplings at a high scale and a consequent reduction of the number of arbitrary parameters. Supersymmetric models postulate the existence of superpartners for all the presently observed particles: bosonic superpartners of fermions (squarks $\tilde{q}$ and sleptons $\tilde{\ell}$ ), and fermionic superpartners of bosons (gluinos $\tilde{g}$ and gauginos $\tilde{\chi}_{i}^{0}, \tilde{\chi}_{i}^{ \pm}$). There are also multiple Higgs bosons: $h, H, A$ and $H^{ \pm}$. There is thus a large spectrum of presently unobserved particles, whose exact masses, couplings and decay chains are calculable in the theory given certain parameters. Unfortunately these parameters are unknown. Nonetheless, if supersymmetry is to have anything to do with electroweak symmetry breaking, the masses should be in the region $100 \mathrm{GeV}-1 \mathrm{TeV}$.

An example of the strong coupling scenario is "technicolor" or models based on dynamical symmetry breaking [23]. Again, if the mechanism is to have anything to do with Electroweak Symmetry breaking we would expect new states in the region $100 \mathrm{GeV}-1 \mathrm{TeV}$; most models predict a large spectrum. An elegant implementation of this appealing idea is lacking. However, all models predict structure in the $W W$ scattering amplitude at around $1 \mathrm{TeV}$ center of mass energy.

There are also other possibilities for new physics that are not necessarily related to the scale of electroweak symmetry breaking. There could be new neutral or charged gauge bosons with mass larger than the $Z$ and $W$; there could be new quarks, charged leptons or massive neutrinos; or quarks and leptons could turn out not to be elementary objects. It is even possible that there are extra space time dimensions [24][25] that have observable consequences for energies in the $\mathrm{TeV}$ mass range. While we have no definitive expectations for the masses of these objects, the LHC must be able to search for them over its available energy range.

\section{THE LARGE HADRON COLLIDER}

\section{A. Machine parameters}

The LHC machine is a proton-proton collider that will be installed in the $26.6 \mathrm{~km}$ circumference tunnel formerly used by the LEP electron-positron collider at CERN [26]. The 8.4 Tesla dipole magnets - each 14.2 meters long (magnetic length) are of the " 2 in 1 " type: the apertures for both beams have a common mechanical structure and cryostat. These superconducting magnets operate at $1.9 \mathrm{~K}$ and have an aperture of $56 \mathrm{~mm}$. They will be placed on the floor in the LEP ring after removal and storage of LEP. The 1104 dipoles and 736 quadruples support beams of $7 \mathrm{TeV}$ energy and a circulating current of 0.54 A.

Bunches of protons separated by $25 \mathrm{~ns}$ and with an RMS length of $75 \mathrm{~mm}$ intersect at four points where experiments are placed. Two of these are high luminosity regions and house the ATLAS and CMS detectors. Two other regions house the ALICE detector [27], to be used for the study of heavy ion collisions, and LHC-B [28], a detector optimized for the study of $b$-mesons and $b$-Baryons. The beams cross at an angle of $200 \mu \mathrm{rad}$, resulting in peak luminosity of $10^{34} \mathrm{~cm}^{-2} s^{-1}$ with a luminosity-lifetime of 10 hours. The expected data samples 
are $30(300) \mathrm{fb}^{-1}$ at $10^{33} \mathrm{~cm}^{-2} s^{-1}\left(10^{34} \mathrm{~cm}^{-2} s^{-1}\right)$, called low (high) luminosity in this document. At the peak luminosity there are an average of $\sim 20 p p$ interactions per bunch crossing. Ultimately, the peak luminosity may increase beyond $10^{34}$ $\mathrm{cm}^{-2} \mathrm{sec}^{-1}$. The machine will also be able to accelerate heavy ions resulting in the possibility of, for example, $\mathrm{Pb}-\mathrm{Pb}$ collisions at $1150 \mathrm{TeV}$ in the center of mass and luminosity up to $10^{27} \mathrm{~cm}^{-2} \mathrm{sec}^{-1}$.

In the $p p$ version, which will be the focus of the rest of this article, the LHC can be thought of as a parton-parton collider with beams of partons of indefinite energy. The effective luminosity [29] of these collisions is proportional to the $p p$ luminosity and falls rapidly with the center of mass energy of the parton-parton system. The combination of the higher energy and luminosity of the LHC compared to the highest energy collider currently operating, the Tevatron, implies that the accessible energy range is extended by approximately a factor of ten.

\section{B. Physics Goals}

The fundamental goal is to uncover and explore the physics behind electroweak symmetry breaking. This involves the following specific challenges:

- Discover or exclude the Standard Model Higgs and/or the multiple Higgs bosons of supersymmetry.

- Discover or exclude supersymmetry over the entire theoretically allowed mass range up to a few $\mathrm{TeV}$.

- Discover or exclude new dynamics at the electroweak scale.

The energy range opened up by the LHC also gives us the opportunity to search for other, possibly less well motivated, objects:

- Discover or exclude any new electroweak gauge bosons with masses below several $\mathrm{TeV}$.

- Discover or exclude any new quarks or leptons that are kinematically accessible.

- Discover or exclude extra-dimensions for which the appropriate mass scale is below several $\mathrm{TeV}$.

Finally we have the possibility of exploiting the enormous production rates for certain Standard Model particles to conduct the following studies:

- Study the properties of the top quark and set limits on exotic decays such as $t \rightarrow c Z$ or $t \rightarrow b H^{+}$.

- Study of $b$-physics, particularly that of $b$-baryons and $B_{s}$ mesons.

An LHC experiment must have the ability to find the unexpected. New phenomena of whatever type will decay into the particles of the Standard Model. In order to cover the lists given above a detector must have great flexibility. The varied physics signatures for these processes require the ability to reconstruct and measure final states involving the following
- Charged leptons, including the $\tau$ via its hadronic decays.

- The electroweak gauge bosons: $W, Z$ and $\gamma$.

- Jets of energy up to several $\mathrm{TeV}$ coming from the production at high transverse momentum of quarks and gluons.

- Jets that have $b$-quarks within them.

- Missing transverse energy carried off by weakly interacting neutral particles such as neutrinos.

In the discussion of physics signals that we present below, it is necessary to estimate production cross sections for both signal and background processes. This is done using perturbative QCD. Such calculations depend on the parton distribution functions that are used, the energy ( $Q^{2}$ scale) used in the evaluation of the QCD coupling constant and the structure functions, and the order in QCD perturbation theory that is used. These issues make comparison between different simulations of the same process difficult. Higher order corrections are not known for all processes and in some cases they are known for the signal and not for the background. Most of the LHC simulations are conservative and use lowest order calculations. Higher order corrections almost always increase the cross sections, typically by a so-called $K$ factor of order 1.5 to 2.0 . The real analysis will of course be based on the best calculations available at the time. At present, the uncertainties from the choice of scale and structure functions are typically at the $20 \%$ level. The total cross-section for $b$-quark production is particularly uncertain.

The level of simulation used to study the processes varies quite widely. For some processes a full GEANT [30] style simulation has been carried out. Such simulations are very slow ( $\sim 10$ Spec95-hr/event) and are difficult to carry out for processes where a large number of events needs to be simulated and many strategies for extracting signals need to be pursued. In these cases a particle level simulation and parameterized detector response is used. A lower level of simulation involving partons (i.e., leptons and jets) and parameterized response is fast and might be required when the underlying parton process is not present in full event generators. This last level of simulation is useful for exploring signals but often leads to overly optimistic results, particularly when the reconstruction of invariant masses of jets or missing energy are involved. None of the results included here use this last level of simulation, unless stated explicitly.

\section{Detectors}

Two large, general-purpose $p p$ collider detectors are being constructed for LHC: ATLAS [1] and CMS [2]. Both collaborations completed Technical Proposals for their detectors in December 1994, and were formally approved in January 1996. Construction is now underway. Though they differ in most details, the detectors share many common features that are derived from the physics goals of LHC:

- they both include precision electromagnetic calorimetry; 
- they both use large magnet systems (though of different geometries) in order to obtain good muon identification and precision momentum measurement;

- they both have lepton identification and precision measurement over $|\eta|<2.5$;

- they both have multi-layer silicon pixel tracker systems for heavy flavor tagging (the usefulness of this capability is an important lesson from the Tevatron);

- they both include calorimetry for large $|\eta|<5$ coverage in order to obtain the required $E_{T}$ resolution.

The ATLAS detector is shown uses a tracking system employing silicon pixels, silicon strip detectors, and a transition radiation tracker, all contained within a 2 Tesla superconducting solenoid. The charged track resolution is $\Delta p_{T} / p_{T}=20 \%$ at $p_{T}=500 \mathrm{GeV}$. The tracker is surrounded by an electromagnetic calorimeter using a lead-liquid argon accordion design; the EM calorimeter covers $|\eta|<3$ (with trigger coverage of $|\eta|<2.5)$ and has a resolution of $\Delta E / E=10 \% / \sqrt{E} \oplus 0.7 \%$. The hadronic calorimeter uses scintillator tiles in the barrel and liquid argon in the endcaps $(|\eta|>1.5)$; its resolution is $\Delta E / E=50 \% / \sqrt{E} \oplus 3 \%$. Forward calorimeters cover the region $3<|\eta|<5$ with a resolution better than $\Delta E / E=$ $100 \% / \sqrt{E} \oplus 10 \%$. Surrounding the calorimeters is the muon system. Muon trajectories are measured using three stations of precision chambers (MDT's and CSC's) in a spectrometer with bending provided by large air-core toroid magnets. The resulting muon momentum resolution is $\Delta p_{T} / p_{T}=8 \%$ at $p_{T}=1 \mathrm{TeV}$ and $\Delta p_{T} / p_{T}=2 \%$ at $p_{T}=100 \mathrm{GeV}$. Muons can be triggered on over the range $|\eta|<2.2$.

The CMS detector is has calorimeters and tracking system are contained in a 4 Tesla superconducting coil which provides the magnetic field for charged particle tracking. The tracking system is based on silicon pixels and silicon strip detectors. The charged track resolution is $\Delta p_{T} / p_{T}=5 \%$ at $p_{T}=1 \mathrm{TeV}$ and $\Delta p_{T} / p_{T}=1 \%$ at $p_{T}=100 \mathrm{GeV}$. CMS has chosen a precision electromagnetic calorimeter using lead tungstate $\left(\mathrm{PbW}_{4}\right)$ crystals, covering $|\eta|<3$ (with trigger coverage of $|\eta|<2.6$ ). Its resolution at low luminosity is $\Delta E / E=3 \% / \sqrt{E} \oplus 0.5 \%$. The surrounding hadronic calorimeter uses scintillator tiles in the barrel and endcaps; its resolution for jets (in combination with the electromagnetic calorimeter) is $\Delta E / E=110 \% / \sqrt{E} \oplus 5 \%$. The region $3<|\eta|<5$ is covered by forward calorimeters using parallel-plate chambers or quartz fibers and having a resolution of about $\Delta E / E=180 \% / \sqrt{E} \oplus 10 \%$. Muon trajectories outside the coil are measured in four layers of chambers (drift tubes and CSC's) embedded in the iron return yoke. The muon momentum measurement using the muon chambers and the central tracker covers the range $|\eta|<2.4$ with a resolution $\Delta p_{T} / p_{T}=5 \%$ at $p_{T}=1 \mathrm{TeV}$ and $\Delta p_{T} / p_{T}=1 \%$ at $p_{T}=100 \mathrm{GeV}$. The muon trigger extends over $|\eta|<2.1$.

\section{HIGGS PHYSICS}

We will use "Higgs bosons" to refer to any scalar particles whose existence is connected to electroweak symmetry break-

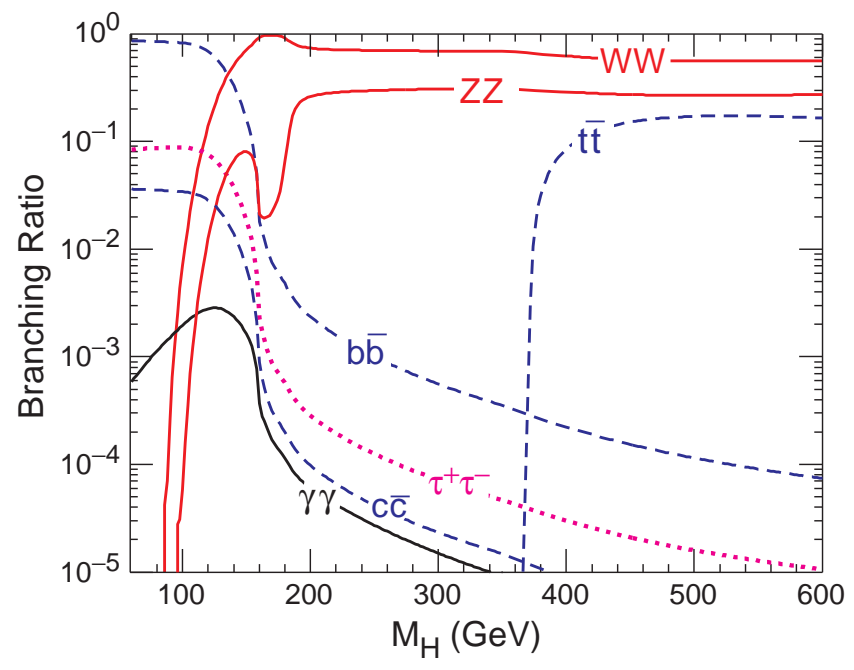

Figure 1: The branching ratios of the Standard Model Higgs boson as a function of its mass.

ing. Generically, Higgs bosons couple most strongly to heavy particles. Their production cross section in hadron colliders is small compared to QCD backgrounds, resulting in final states with low rates or low signal-to-background ratios. The ability to detect them and measure their mass provides a set of benchmarks by which detectors can be judged. A specific model is required in order to address the quantitative questions of how well the detector can perform. While one may not believe in the details of any particular model, a survey of them will enable general statements to be made about the potential of the LHC and its detectors.

\section{A. Standard Model Higgs}

All the properties of the Standard Model Higgs boson are determined once its mass is known; the search strategy at LHC is therefore well defined. The current limit on the Higgs mass from experiments at LEP [19] is $M_{H}>113.5 \mathrm{GeV}$. There are several relevant production mechanisms at LHC; $g g \rightarrow H$ via a heavy quark loop; $q \bar{q} \rightarrow W H ; g g \rightarrow t \bar{t} H ; g g \rightarrow b \bar{b} H$ and $q q \rightarrow q q H$ (" $W W$ fusion"). The relative importance of these processes depends upon the Higgs mass, the first dominates at small mass and the two become comparable for a Higgs mass of $1 \mathrm{TeV}$. The Higgs branching ratios are shown in Fig. 11.

\section{1. $H \rightarrow \gamma \gamma$ and associated production channels}

At masses just above the range probed by LEP, the dominant decay of the Higgs boson is to $b \bar{b}$, which is essentially impossible to separate from the huge QCD $b \bar{b}$ background. The decay to $\gamma \gamma$ is the most promising channel in this region. The branching ratio is very small, and there is a large background from the pair production of photons via $q \bar{q} \rightarrow \gamma \gamma, g g \rightarrow \gamma \gamma$, and the bremsstrahlung process $q g \rightarrow q(\rightarrow \gamma) \gamma$. Excellent photon energy resolution is required to observe this signal. Hence, this process is one that drives the very high quality electromagnetic calorimetry of both ATLAS and CMS. 
CMS has a mass resolution of $540(870) \mathrm{MeV}$ at $m_{H}=$ $110 \mathrm{GeV}$ for low (high) luminosity [31]. The mass resolution is worse at high luminosity due to event pile up. The ATLAS mass resolution at low (high) luminosity is 1.1 (1.3) GeV for $M_{H}=110 \mathrm{GeV}$. The photon acceptance and identification efficiency are higher in the ATLAS analysis [32], partly because CMS rejects some of the photons that convert in the inner detector.

In addition to the background from $\gamma \gamma$ final states, there are jet $-\gamma$ and jet - jet final states, that are much larger. A jet $/ \gamma$ rejection factor of $\sim 10^{3}$ is needed to bring these backgrounds below the irreducible $\gamma \gamma$ background. A detailed GEANT based study of the ATLAS detector has been performed for these backgrounds [32]. Jets were rejected by applying cuts on the hadronic leakage, isolation and the measured width of the electromagnetic shower. These cuts result in an estimate of these backgrounds which is a factor of four below the irreducible $\gamma \gamma$ background. There are uncertainties in the rates for these "reducible" backgrounds, however one can be confident that they are smaller after cuts than the irreducible $\gamma \gamma$ background.

In the CMS analysis for this process [2, 31], two isolated photons are required, one with $p_{T}>25 \mathrm{GeV}$ and the other with $p_{T}>40 \mathrm{GeV}$. Both are required to satisfy $|\eta|<2.5$ and to have no track or additional electromagnetic energy cluster with $p_{T}>2.5 \mathrm{GeV}$ in a cone of size $\Delta R=0.3$ around the photon direction. The Higgs signal then appears as a peak over the smooth background. The signal-to-background ratio is small, but there are many events. A curve can be fitted to the smooth background and subtracted from data. Fig. 4 shows the total and background-subtracted distributions for a Higgs mass of $130 \mathrm{GeV}$. For an integrated luminosity of $100 \mathrm{fb}^{-1}$ it is possible to discover a Higgs using this mode if its mass is between the lower limit set by LEP and about $140 \mathrm{GeV}$. A signal can also be found over a more limited mass range for an integrated luminosity of $10 \mathrm{fb}^{-1}$. Results of the ATLAS study are similar [32].

Another process is available at the lower end of the mass range. If the Higgs is produced in association with a $W$ or $t \bar{t}$, the cross section is substantially reduced, but the presence of additional particles provide a proportionally larger reduction in the background. Events are required to have an isolated lepton arising from the decay of the $W$ (or top quark). This lepton can be used to determine the vertex position. The process is only useful at high luminosity as, for $10 \mathrm{fb}^{-1}$, there are approximately 15 signal events for Higgs masses between 90 and $120 \mathrm{GeV}$ (the falling cross-section is compensated by the increased branching ratio for $H \rightarrow \gamma \gamma$ ) over an approximately equal background [35]. The process will therefore provide confirmation of a discovery made in the $\gamma \gamma$ final state without an associated lepton and measurements of the couplings.

$$
\text { 2. } H \rightarrow b \bar{b}
$$

The dominant decay of a Higgs boson if its mass is below $2 M_{W}$ is to $b \bar{b}$. The signal for a Higgs boson produced in isolation is impossible to extract: there is no trigger for the process and the background production of $b \bar{b}$ pairs is enormous. The production of a Higgs boson in association with a $W$ or $t \bar{t}$ pair
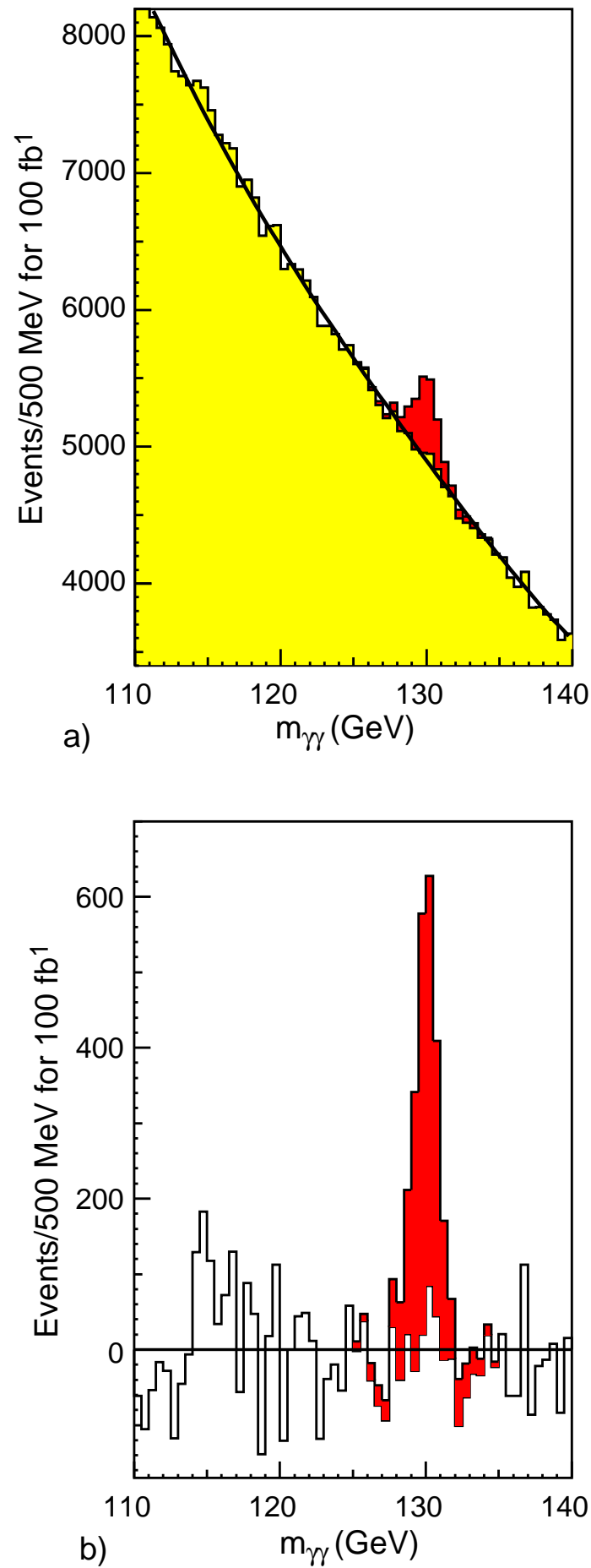

Figure 2: (a) The invariant mass distribution of $\gamma \gamma$ pairs for $M_{h}=130 \mathrm{GeV}$ as simulated by the CMS collaboration. (b) Same, with a smooth background fitted and subtracted. From Ref. [40].

can provide a high $p_{T}$ lepton that can be used as a trigger. A study was conducted by ATLAS of this very challenging channel (see Section of 19.2.4 of Ref. [35]). Events were triggered by requiring a muon (electron) with $|\eta|<2.5$ and $p_{T}>6(20)$ $\mathrm{GeV}$. 


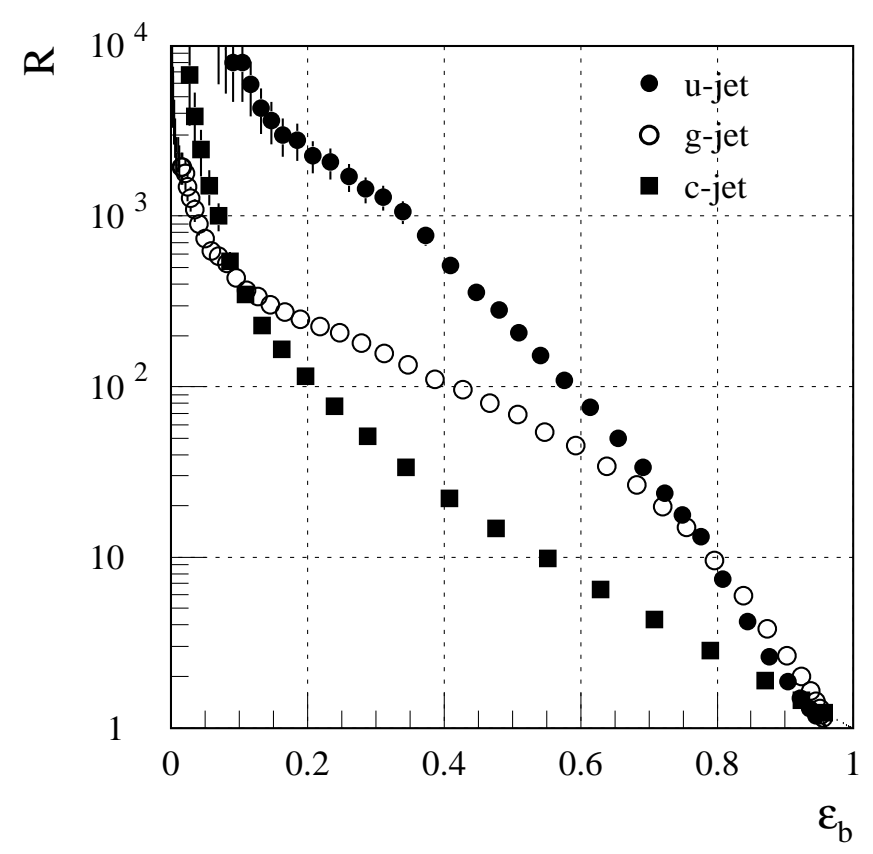

Figure 3: Rejection factor for jets produced from $u$ and $c$ quarks and gluons at low luminosity as a function of the tagging efficiency for $b$-quark jets in the ATLAS detector. Processes such as $g \rightarrow b \bar{b}$ are included as mistags. From Ref. [35].

The expected $b$-tagging efficiency for ATLAS was determined by full simulation of samples of $H \rightarrow b \bar{b}, H \rightarrow u \bar{u}$, and $t \bar{t}$ events. The results from these samples for the $b$-tagging rate and rate of fake tags from non $b$-jets were obtained over a range of $p_{T}$. The results can therefore be extrapolated to other cases e.g, $b$-jets in supersymmetry events - that have not been fully simulated. The ATLAS detector has a pixel layer at $\sim 5 \mathrm{~cm}$ from the beam. The $b$-tagging efficiency is correlated with the rejection factor that is obtained against other jets as is shown in Fig. 3. The rejection of charm jets is limited by the lifetime of charged hadrons and that of gluons by the production of $b \bar{b}$ pairs in the jet itself. Note that rejection factors $\sim 100$ against light quark jets can be obtained for a $b$-tagging efficiency of $60 \%$. The $b$-tagging efficiency for CMS has similarly been determined from full simulation and is shown in Figure 4 . As in the case of ATLAS the pixel layers at radii of 4,7 , and $11 \mathrm{~cm}$ are used for the tagging. These $b$-tagging efficiencies are not significantly different from those already obtained by CDF [33.

The ATLAS study of $H \rightarrow b \bar{b}$ uses the tagging efficiencies from the full simulation study. Both the $t \bar{t} H$ and $W H$ final states were studied but the former is more powerful, so it is the only one discussed here. Jets were retained if they had $p_{T}>15$ $\mathrm{GeV}$. This threshold was raised to $30 \mathrm{GeV}$ for simulations at a luminosity of $10^{34} \mathrm{~cm}^{-2} \mathrm{sec}^{-1}$. In order to reduce the background a veto was applied to reject events with a second isolated lepton $p_{T}>6 \mathrm{GeV}$ and $|\eta|<2.5$ and a total of 4 tagged $b$-jets was required. Reconstruction of both top quarks using a kinematic fit is essential to reduce the combinatorial $b \bar{b}$ background. For a luminosity of $100 \mathrm{fb}^{-1}$, there are 107 and 62 signal events for Higgs masses of 100 and $120 \mathrm{GeV}$. The reconstructed $b \bar{b}$

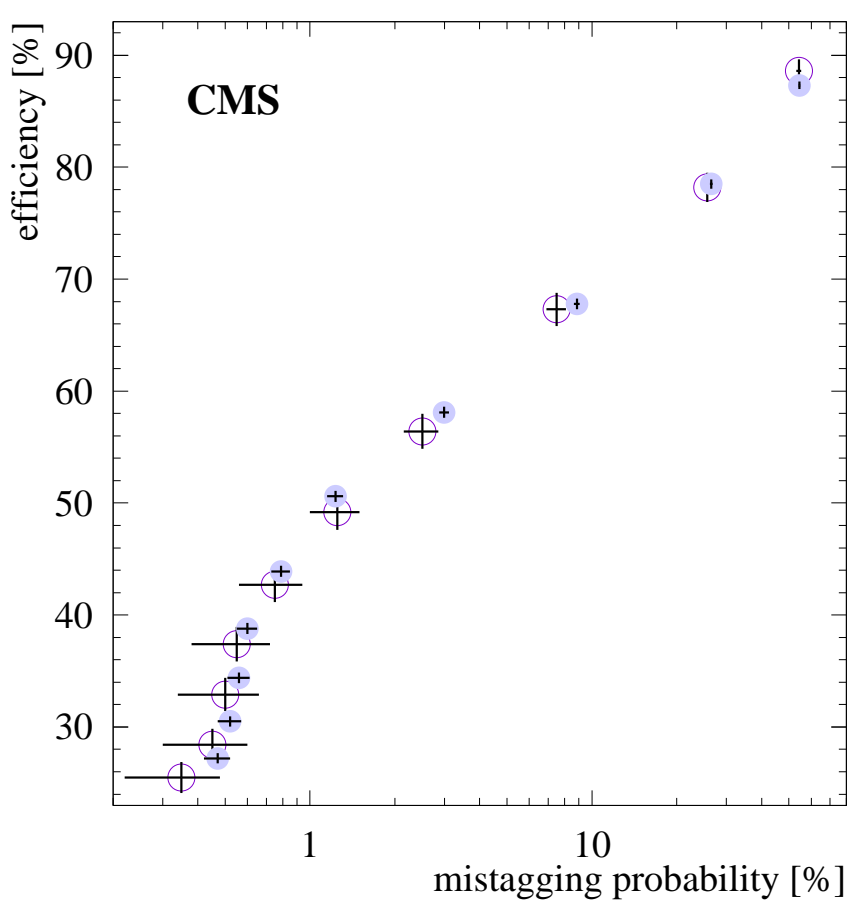

Figure 4: Mistagging probability for jets produced from $u$ quarks as a function of the tagging efficiency for $b$-quark jets in the CMS detector with the all silicon tracker. From Ref. [34].

mass distribution is approximately Gaussian with $\sigma / M \sim 0.2$; it has a tail on the low side caused mainly by gluon radiation off the final state $b$ quarks and losses due to decays. The background arising from $t \bar{t} j j$ events is the most important; approximately 250 events in a bin of width $30 \mathrm{GeV}$ centered on the reconstructed $b \bar{b}$ mass peak. Fig. 5 shows the reconstructed $b \bar{b}$ mass distributions for the summed signal and background for $m_{H}=120 \mathrm{GeV}$. Extraction of a signal will be possible if at all only over a limited mass range $(\sim 80-120 \mathrm{GeV})$ and depends critically upon the $b$-tagging efficiency and background rejection. The signal will provide a second observation of the Higgs boson in this mass range and also provide valuable information on the Higgs couplings.

A similar analysis has been performed by CMS [36]. Events were required to have an isolated $e$ or $\mu$ with $p_{T}>10 \mathrm{GeV}$, six jets with $p_{T}>20 \mathrm{GeV}$, four of which are tagged as $b$ 's. A K-factor of 1.5 is included for the signal only. A likelihood analysis gave the results shown in Fig. 6 with $S / B=0.73$. The extraction of the $t \bar{t} H$ Yukawa coupling from this signal was also studied.

$$
\text { 3. } H \rightarrow Z Z^{*} \rightarrow 4 \ell
$$

The search for the Standard Model Higgs relies on the fourlepton channel over a broad mass range from $m_{H} \sim 130 \mathrm{GeV}$ to $m_{H} \sim 800 \mathrm{GeV}$. Below $2 m_{Z}$, the event rate is small and the background reduction more difficult, as one or both of the $Z$-bosons are off-shell. In this mass region the Higgs width is small $(\lesssim 1 \mathrm{GeV})$ and so lepton energy or momentum resolu- 


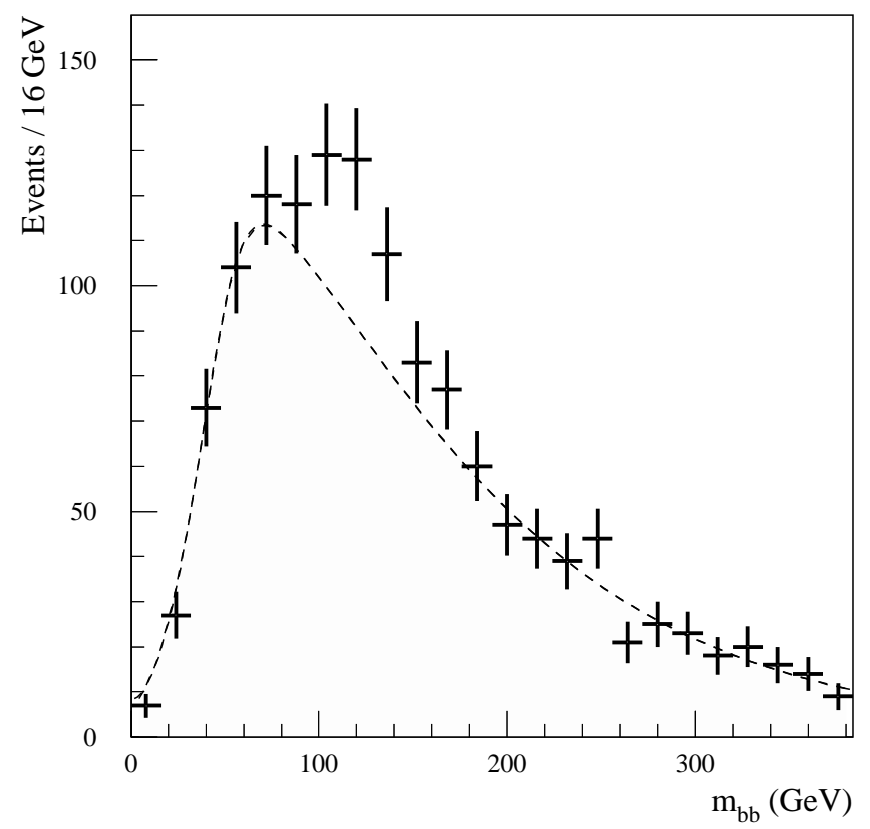

Figure 5: Invariant mass distribution, $m_{b \bar{b}}$, of tagged $b$-jet pairs in fully reconstructed $t \bar{t} H$ signal events with a Higgs-boson mass of $120 \mathrm{GeV}$ above the summed background (see text), for an integrated luminosity of $100 \mathrm{fb}^{-1}\left(30 \mathrm{fb}^{-1}\right.$ with lowluminosity operation and $70 \mathrm{fb}^{-1}$ with high-luminosity operation). The points with error bars represent the result of a single experiment and the dashed line represents the background distribution. From Ref. [35].

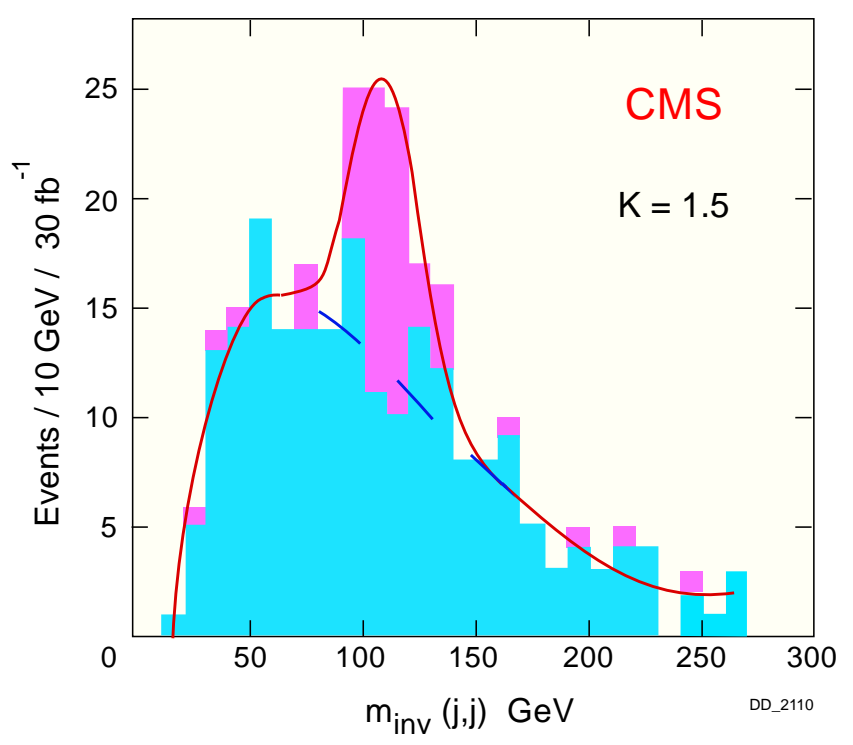

Figure 6: Reconstructed mass distribution, $m_{j, j}$, of tagged $b$ jet pairs showing a $t \bar{t} H$ signal with a Higgs-boson mass of $115 \mathrm{GeV}$ above the background, for an integrated luminosity of $30 \mathrm{fb}^{-1}$. From Ref. [36].
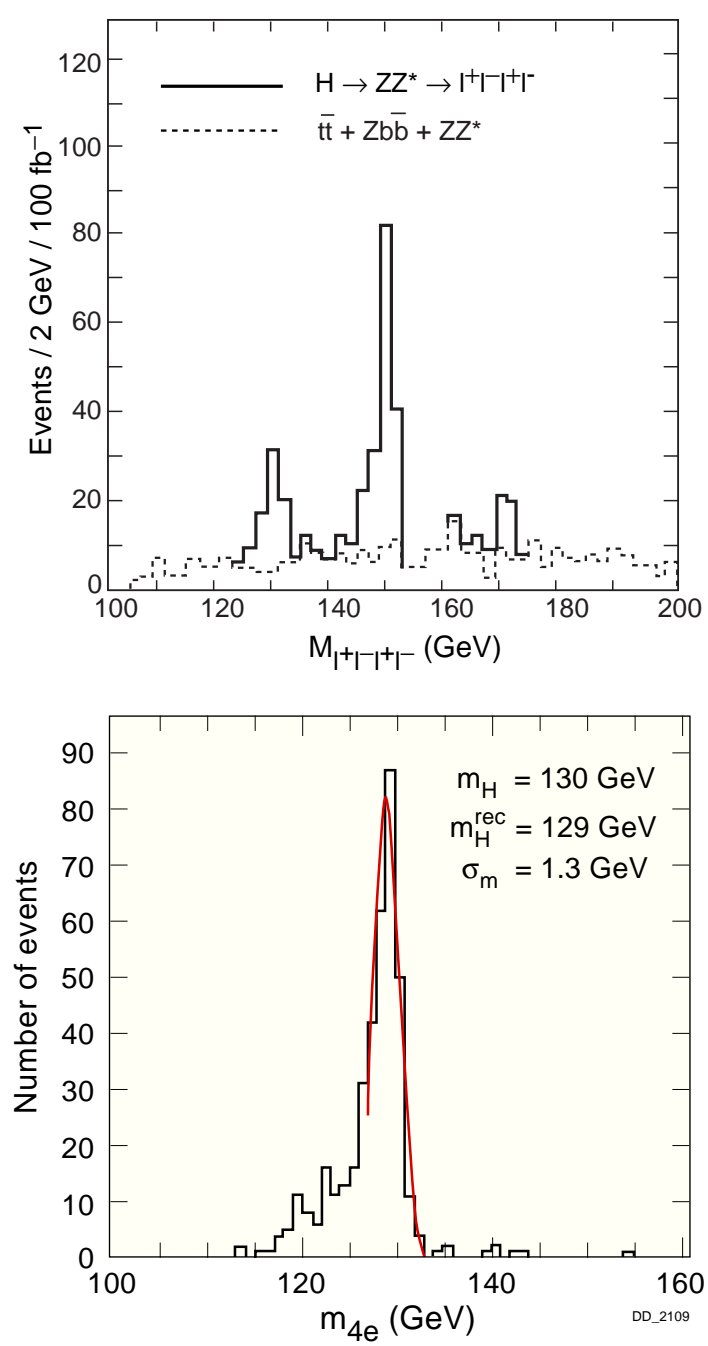

Figure 7: Top: Reconstructed four-lepton mass in CMS for $H \rightarrow 4 \ell$ for various masses and sum of all backgrounds with $100 \mathrm{fb}^{-1}$. Bottom: Reconstructed four-electron mass for $m_{H}=130 \mathrm{GeV}$, showing the radiative tail predicted by full simulation. From Ref. [41].

tion is of great importance in determining the significance of a signal [37].

For $m_{H}<2 m_{Z}$, the main backgrounds arise from $t \bar{t}, Z b \bar{b}$ and continuum $Z Z^{*} / Z \gamma^{*}$ production. Of these, the $t \bar{t}$ background can be reduced by lepton isolation and by lepton pair invariant mass cuts. The $Z b \bar{b}$ background cannot be reduced by a lepton pair invariant mass cut but can be suppressed by isolation requirements and impact parameter cuts. The $Z Z^{*}$ process is an irreducible background. Both CMS and ATLAS studied the process for $m_{H}=130,150$ and $170 \mathrm{GeV}$. Signal events were obtained from both $g g \rightarrow H$ and $W W / Z Z$ fusion processes, giving consistent cross sections $\sigma \cdot B \approx 3,5.5$ and $1.4 \mathrm{fb}$ respectively (no $K$-factors being included).

In the CMS study [2, 38] event pileup appropriate to $\mathcal{L}=$ $10^{34} \mathrm{~cm}^{-2} \mathrm{~s}^{-1}$ was modeled by superimposing 15 minimum bias events (simulated by QCD dijets with $p_{T} \geq 5 \mathrm{GeV}$ ). The muon resolution was obtained from a full simulation of the de- 
tector response and track-fitting procedure. This was then parameterized as a function of $p_{T}$ and $\eta$. Internal bremsstrahlung was generated using the PHOTOS program and leads to about $8 \%$ of reconstructed $Z \rightarrow \mu^{+} \mu^{-}$pairs falling outside a $m_{Z} \pm$ $2 \sigma_{Z}$ window for $m_{H}=150 \mathrm{GeV}$. The reconstructed $\mu^{+} \mu^{-}$ mass has a resolution $\sigma_{Z}=1.8 \mathrm{GeV}$ in the Gaussian part of the peak. The electron response was obtained from a full GEANT simulation of the calorimeter, including the effects of material in the beampipe and the tracker, and the reconstruction of electron energy in the crystal calorimeter. Including internal and external bremsstrahlung, and using a $5 \times 7$ crystal matrix to reconstruct the electron, the mass resolution $\sigma_{Z}=2.5 \mathrm{GeV}$ and the reconstruction efficiency is about $70 \%$ (within $m_{Z} \pm 2 \sigma_{Z}$ ).

Events were selected which had one electron with $p_{T}>$ $20 \mathrm{GeV}$, one with $p_{T}>15 \mathrm{GeV}$ and the remaining two with $p_{T}>10 \mathrm{GeV}$, all within $|\eta|<2.5$. For muons, the momenta were required to exceed 20,10 and $5 \mathrm{GeV}$ within $|\eta|<2.4$. One of the $e^{+} e^{-}$or $\mu^{+} \mu^{-}$pairs was required to be within $\pm 2 \sigma_{Z}$ of the $Z$ mass. This cut loses that fraction of the signal where both $Z$ 's are off-shell, about a $24 \%$ inefficiency at $m_{H}=130 \mathrm{GeV}$ and $12 \%$ at $m_{H}=170 \mathrm{GeV}$. The two softer leptons were also required to satisfy $m_{\ell \ell}>12 \mathrm{GeV}$. Additional rejection is obtained by requiring that any three of the four leptons be isolated in the tracker, demanding that there is no track with $p_{T}>2.5 \mathrm{GeV}$ within the cone $R<0.2$ around the lepton. This requirement is not very sensitive to pileup as the $2.5 \mathrm{GeV}$ threshold is quite high. This yields signals at the level of 7.4, 15.2 and 5.0 standard deviations for $m_{H}=130,150$, and $170 \mathrm{GeV}$ in $200 \mathrm{fb}^{-1}$. The four-lepton mass distributions are shown in Fig. 7 which also shows the $4 e$ final state. The latter clearly shows the effect of bremsstrahlung.

The ATLAS [35] study followed a similar technique. The detector resolutions and reconstruction efficiencies were obtained using detailed detector simulations, including the effects of pileup. Events were selected which had two leptons with $p_{T}>20 \mathrm{GeV}$, and the remaining two with $p_{T}>7 \mathrm{GeV}$, all within $|\eta|<2.5$. One of the $e^{+} e^{-}$or $\mu^{+} \mu^{-}$pairs was required to be within $\pm m_{12}, \mathrm{GeV}$ of the $Z$ mass. The two softer leptons were also required to satisfy $m_{\ell \ell}>m_{34} . m_{12}$ and $m_{34}$ are varied as a function of the Higgs mass; for $m_{H}=130 \mathrm{GeV}$, $m_{12}=10 \mathrm{GeV}$ and $m_{34}=30 \mathrm{GeV}$. For the four-electron mode, the Higgs mass resolution at $m_{H}=130 \mathrm{GeV}$ is $1.8(1.5) \mathrm{GeV}$ at high (low) luminosity, including the effect of electronic noise in the calorimeter. For muons, the corresponding figure is $1.4 \mathrm{GeV}$ after correcting for muon energy losses in the calorimeter and combining the muon momentum measured in the muon system with that obtained from the central tracker after the tracks have been matched.

ATLAS used a combination of calorimeter isolation and impact parameter cuts to reject background from $Z b \bar{b}$ and $t \bar{t}$ events. The isolation criterion is that the transverse energy within $R=0.2$ of the lepton be less than $E_{T}^{c u t}$ or that there are no additional reconstructed tracks above a threshold in the cone. The rejections obtained by these methods are correlated. Values of $E_{T}^{c u t}$ of 3,5 , and $7 \mathrm{GeV}$ were used for $4 \mu$, eе $\mu \mu$ and $4 e$ modes at $10^{33}\left(10^{34}\right)$ luminosity to obtain a constant signal efficiency of $85 \%(50 \%)$. Tighter cuts can be used for muons

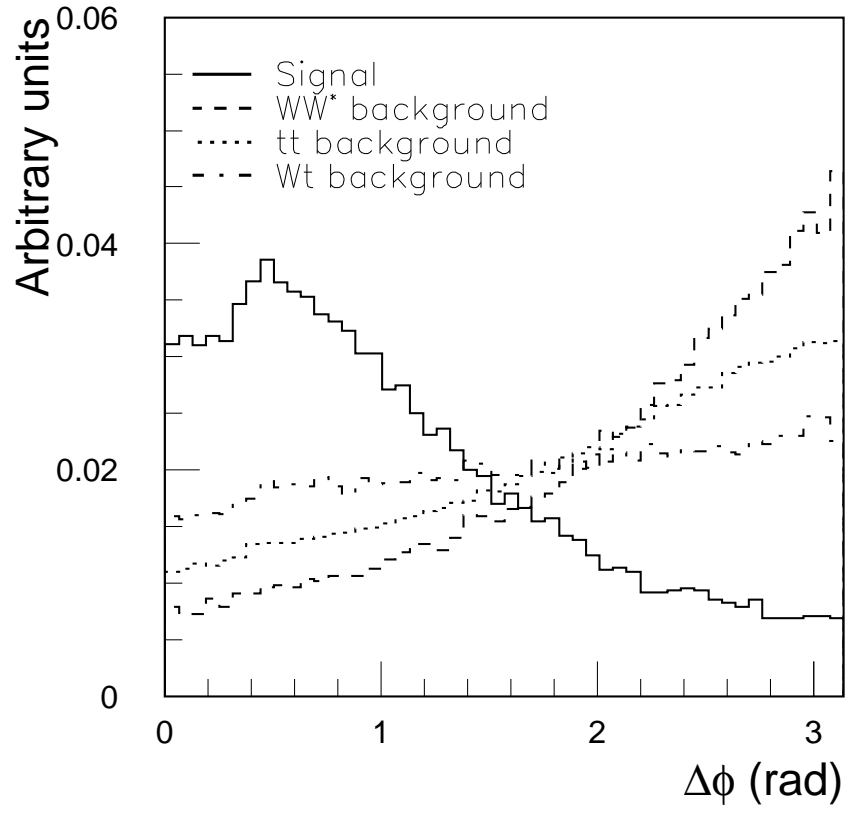

Figure 8: Difference in azimuth between the two leptons for $H \rightarrow W W^{*} \rightarrow \ell \nu \ell \nu$ signal events with $m_{H}=170 \mathrm{GeV}$ and for the $W W^{*}, t \bar{t}$ and $W t$ background events. All distributions are normalized to unity.From Ref. 35.

because they do not suffer from transverse leakage of the EM shower. The impact parameter, as measured in the pixel layers, is used to further reduce the background from heavy flavor processes $(t \bar{t}$ and $Z b \bar{b})$ [35]. ATLAS obtain signals at the level of 10.3 (7.0), 22.6 (15.5) and 6.5 (4.3) standard deviations for $m_{H}=130,150$, and $170 \mathrm{GeV}$ in $100 \mathrm{fb}^{-1}\left(30 \mathrm{fb}^{-1}\right)$.

$$
\text { 4. } H \rightarrow W W^{(*)} \rightarrow \ell^{+} \nu \ell^{-} \bar{\nu}
$$

The decay $H \rightarrow W W^{(*)} \rightarrow \ell^{+} \nu \ell^{-} \bar{\nu}$ can provide valuable information in the mass region around $170 \mathrm{GeV}$ where the branching ratio $H \rightarrow 4 \ell$ is reduced [42]. For this mass the two-body $W W$ decay dominates, so $B R\left(H \rightarrow W W^{(*)} \rightarrow\right.$ $\left.\ell^{+} \nu \ell^{-} \bar{\nu}\right) / B R(H \rightarrow 4 \ell) \sim 100$.

For the $\ell^{+} \nu \ell^{-} \bar{\nu}$ final state, the Higgs mass cannot be reconstructed, so the signal must be observed from an excess of events. The dominant background arises from the production of $W$ pairs after cuts to remove the $t \bar{t}$ background. The ATLAS analysis [35] requires:

- Two isolated opposite sign leptons with $|\eta|<2.5$ and $p_{T}>20,10 \mathrm{GeV}$. In addition the pair must satisfy $M_{\ell \ell}<$ $80 \mathrm{GeV}, \Delta \phi_{\ell \ell}<1$, and $\Delta \eta_{\ell \ell}<1.5$.

- No jets with $p_{T}>15 \mathrm{GeV}$ and $|\eta|<3.1$.

- $\mathbb{E}_{T}>40 \mathrm{GeV}$.

- A $\ell \ell \mathbb{E}_{T}$ transverse mass between $m_{H}-30 \mathrm{GeV}$ and $m_{H}$.

At luminosity of $10^{34} \mathrm{~cm}^{-2} \mathrm{sec}^{-1}$, the jet veto is raised to $30 \mathrm{GeV}$. After these cuts the signal to background ratio is approximately 2:1 and there are 340 signal events for $m_{H}=$ 

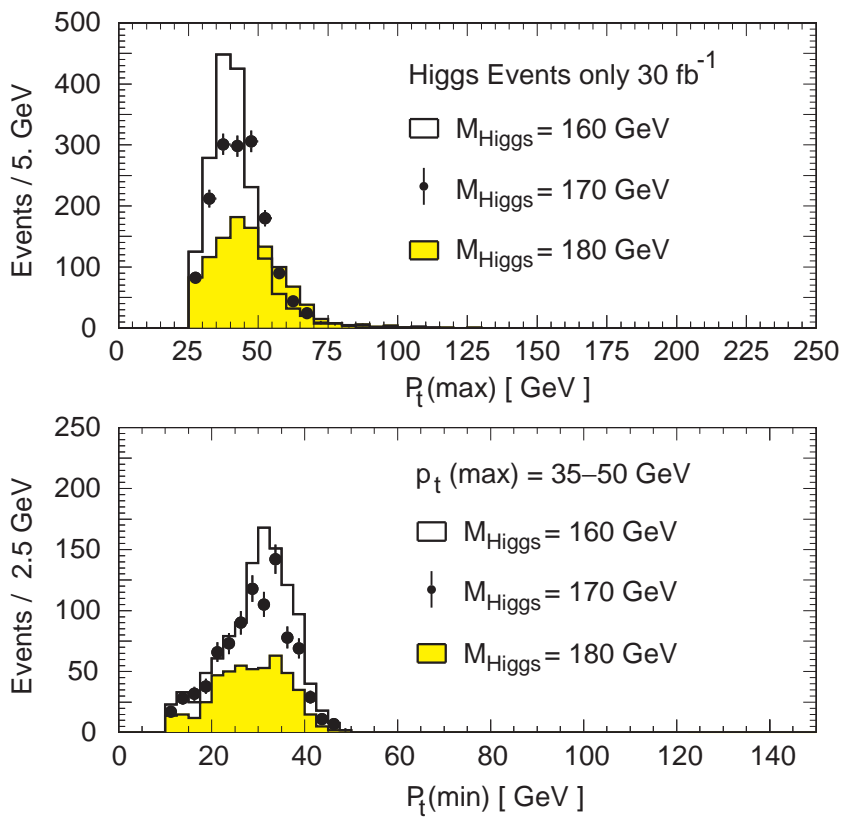

Figure 9: Dependence of the lepton kinematics for the $H \rightarrow$ $W W \rightarrow \ell^{+} \nu \ell^{-} \bar{\nu}$ signal on the Higgs mass. From Ref. [44].

$170 \mathrm{GeV}$ for $30 \mathrm{fb}^{-1}$. The signal can be clearly established by looking at the distribution in the azimuthal separation of the leptons $(\Delta \phi)$. As is shown in Fig. 8, this is peaked at small (large) values of $\Delta \phi$ for the signal (background).

Some information on the Higgs mass can be obtained from the lepton kinematics. This is shown in Fig. 9 from a CMS analysis. This figure shows the distribution of the larger and smaller $p_{T}$ 's of the two leptons for an assumed mass of $170 \mathrm{GeV}$ in comparison with the expectation for masses of $160 \mathrm{GeV}$ and $180 \mathrm{GeV}$.

As the Higgs mass falls significantly below $150 \mathrm{GeV}$ the event rate becomes small. The observability of the signal depends crucially on the ability to correctly predict the background. This background estimation can be checked by comparison with the $Z Z$ final state and by measuring the $W W$ system away from the signal region. A 5\% systematic error on the background can be expected; then a $5 \sigma$ observation can be made in the range $130 \mathrm{GeV}<m_{H}<190 \mathrm{GeV}$ for $30 \mathrm{fb}^{-1}$ of integrated luminosity [35],

$$
\text { 5. } H \rightarrow Z Z \rightarrow 4 \ell
$$

The $H \rightarrow Z Z \rightarrow 4 \ell$ channel is sensitive over a wide range of Higgs masses from $2 m_{Z}$ upwards: to about $400 \mathrm{GeV}$ with $10 \mathrm{fb}^{-1}$ and to about $600 \mathrm{GeV}$ with $100 \mathrm{fb}^{-1}$. For lower Higgs masses, the width is quite small and precision lepton energy and momentum measurements are helpful; for larger masses the natural Higgs width becomes large. The main background is continuum $Z Z$ production.

CMS [2 38] studied the process for $m_{H}=300,500$ and $600 \mathrm{GeV}$. The electron and muon resolutions and the selection cuts were the same as used for the $Z Z^{*}$ channel. Two $e^{+} e^{-}$ or $\mu^{+} \mu^{-}$pairs with a mass within $\pm 6 \mathrm{GeV}$ of $m_{Z}$ were required. No isolation cut was imposed as the remaining back-
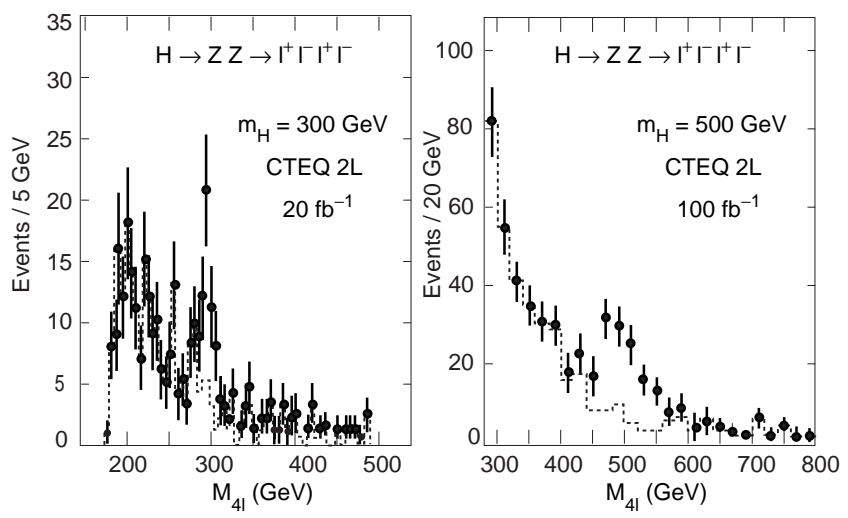

Figure 10: Mass distribution in $H \rightarrow Z Z \rightarrow 4 \ell$ for various values of $m_{H}$ as simulated by CMS including all bremsstrahlung losses. From Ref. [43].

grounds are small. The resulting 4-lepton invariant mass distributions are shown in Fig. 10. With $100 \mathrm{fb}^{-1}$ a signal in excess of six standard deviations is visible over the entire range $200<m_{H}<600 \mathrm{GeV}$. ATLAS obtains very similar results [35].

$$
\text { 6. } M_{H} \sim 1 \operatorname{TeV}(H \rightarrow \ell \ell \nu \nu, \ell \ell j j, \ell \nu j j)
$$

As the Higgs mass is increased further, its width increases and its production rate falls, so one must turn to decay channels that have a larger branching ratio. The first of these is $H \rightarrow Z Z \rightarrow \ell \ell \nu \bar{\nu}$. Here the signal involves a $Z$ decaying to lepton pairs and a large amount of missing energy. The signal appears as a Jacobian peak in the missing $E_{T}$ spectrum. There are more potentially important sources of background in this channel than in the $4 \ell$ final state. In addition to the irreducible background from $Z Z$ final states, one has to worry about $Z+$ jets events where the missing $E_{T}$ arises from neutrinos in the jets or from cracks and other detector effects that cause jet energies to be mismeasured. At high luminosity the background from the pile up of minimum bias events produces a $\mathbb{E}_{T}$ spectrum that falls very rapidly and is small for $\mathbb{E}_{T}>100$ $\mathrm{GeV}$, provided the calorimeter extends to $|\eta|<5$. ATLAS conducted [45] a full GEANT based study of this background for which 5000 high transverse momentum $Z+$ jet events were fully simulated. The events were selected so that a large fraction of them had jets going into the region $0.9<|\eta|<1.3$ where ATLAS has weaker jet energy resolution due to the crack between the endcap and barrel hadron calorimeters. The dominant part of the $Z+$ jets background that remains is that where the missing $E_{T}$ arises from the semi-leptonic decays of $b$-quarks in the jets. The contribution from detector effects is not dominant.

Fig. 11 shows the missing $E_{T}$ spectrum at high luminosity $\left(100 \mathrm{fb}^{-1}\right)$. On this plot the $Z+$ jets background is estimated from a parton level simulation; there were insufficient statistics in the full study to obtain this spectrum. This estimate correctly models the contribution from $b$-decays that the full study showed to be dominant. The reconstructed $Z \rightarrow \ell \ell$ was re- 


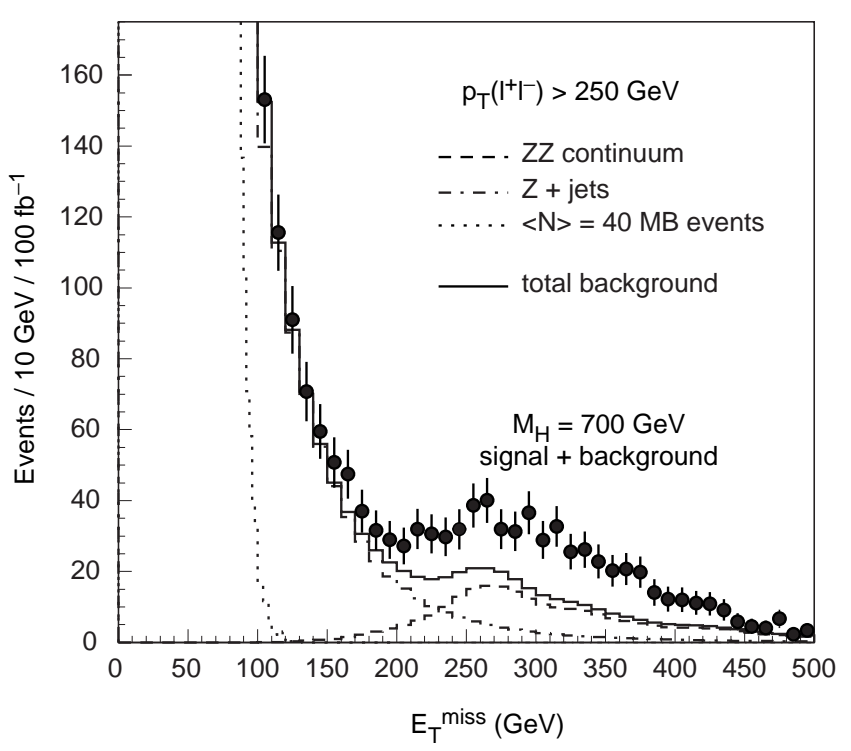

Figure 11: Missing $E_{T}$ spectrum for the $H \rightarrow Z Z \rightarrow \ell \ell \nu \bar{\nu}$ process. The background contributions are shown separately; $Z+$ jets (dot-dashed); ZZ (dotted) and minimum bias pile up (dashed). The signal is due to a Higgs boson of mass $700 \mathrm{GeV}$. From Ref. [1].

quired to have $p_{T}(Z)>250 \mathrm{GeV}$; this causes the $Z Z$ background to peak. (This effect is less pronounced if a cut is made on $\mathbb{E}_{T}$ and then the plot is remade with $p_{T}(Z)$ on the abscissa.) The dominant $Z Z$ background has QCD corrections of order $40 \%$ [46]. Once data are available, this background will be measured. It signal to background ratio can be improved significantly by requiring one or two forward jets at the cost of a smaller acceptance [35].

The CMS analysis of this process [2, 47] uses a central jet veto, requiring that there be no jets with $E_{T}>150 \mathrm{GeV}$ within $|\eta|<2.4$. By requiring a jet in the far forward region (see below), most of the remaining $Z Z$ background can be rejected. A study by CMS requiring a jet with $E>1 \mathrm{TeV}$ and $2.4<$ $|\eta|<4.7$, produces an improvement of approximately a factor of three in the signal to background ratio at the cost of some signal. This mode is only effective for high mass Higgs bosons and becomes powerful only at high luminosity. Nevertheless it will provide an unambiguous signal.

Substantially larger event samples are available if the decay modes $H \rightarrow W W \rightarrow \ell \nu+$ jets and $H \rightarrow Z Z \rightarrow \ell \ell+$ jets can be exploited efficiently. In order to do this one has to reduce the enormous $W+$ jets and $Z+$ jets backgrounds by kinematic cuts. Henceforth the discussion will be for the $W W$ final state; the $Z Z$ state is similar. The first step is to reconstruct the $W$ decay to jets. Full and fast simulations of the ATLAS detector were used and are in good agreement [35]. At large values of $m_{H}$ the jets from the $W$ decay tend to overlap and several methods were used to reconstruct the $W$. In one method, jets were found using a cone of size $\Delta R=0.2$ and $E_{T}>50 \mathrm{GeV}$. The invariant mass of the di-jet system was then computed by adding the four-momenta of the calorimeter cells assuming that
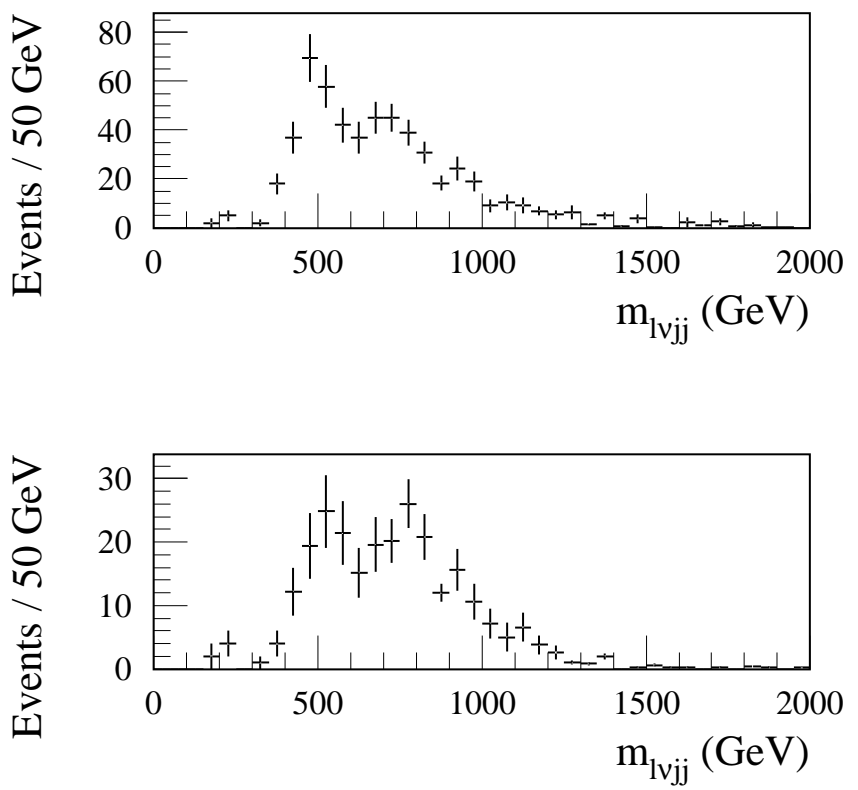

Figure 12: For an integrated luminosity of $30 \mathrm{fb}^{-1}$ and for $m_{H}=800 \mathrm{GeV}$, distribution of $M_{l \nu j j}$ in ATLAS for the summed signal + background after requiring two tag jets with $E_{\text {tag }}>200 \mathrm{GeV}$ (top) and $E_{\text {tag }}>400 \mathrm{GeV}$ (bottom). From Ref. [35].

Table I: $H \rightarrow W W \rightarrow \ell \nu j j$ signals and backgrounds, for $m_{H}=1 \mathrm{TeV}$, before and after cuts in the forward region (see text). The rates are computed for an integrated luminosity of $30 \mathrm{fb}^{-1}$ and a lepton efficiency of $90 \%$. Only the $q q \rightarrow H q q$ contribution to the signal is included. Table from ATLAS simulation.

\begin{tabular}{|l|r|r|r|}
\hline Process & $\begin{array}{r}\text { Central } \\
\text { cuts }\end{array}$ & $\begin{array}{r}\text { Jet } \\
\text { veto }\end{array}$ & $\begin{array}{r}\text { Double } \\
\text { tag }\end{array}$ \\
\hline \hline$H \rightarrow W W$ & 222 & 143 & 73 \\
\hline$t \bar{t}$ & 38300 & 2800 & 85 \\
\hline$W+$ jets & 15700 & 6900 & 62 \\
\hline
\end{tabular}

each cell is massless. The di-jet system is required to have $E_{T}>150 \mathrm{GeV}$ This algorithm reconstructs $W \rightarrow$ jets with an efficiency of about $60 \%$ and a $W$ mass resolution of $6.9 \mathrm{GeV}$ for $W^{\prime} s$ produced in the decay of $1 \mathrm{TeV}$ Higgs bosons. The mass resolution improves to $5 \mathrm{GeV}$ at low luminosity where pile up is unimportant. The dijet system is then required to have a mass within $2 \sigma$ of the nominal $W$ mass. In addition the events are required to have a lepton with $p_{T}>50 \mathrm{GeV}$ and $\mathbb{E}_{T}>50 \mathrm{GeV}$. These cuts applied to the $W(\rightarrow \ell \nu)+$ jets sample with $p_{T}(W)>200 \mathrm{GeV}$ reduce the rate for this process by a factor of 600 and brings it to a level approximately equal to that from $t \bar{t}$ production; $t \bar{t} \rightarrow W b W \bar{b}$.

After these cuts, the backgrounds from $W+$ jets and $t \bar{t}$ are still larger than the signal from $H \rightarrow W W$ and topological cuts are required. The process $q q \rightarrow H q q$ produces the Higgs boson in association with jets at large rapidity. These jets can be used 
as a tag to reject background. This forward jet tag will cause some loss of signal since the $g g \rightarrow H$ process lacks these forward jets. Hence it is only effective for high mass Higgs bosons where the $q q \rightarrow H q q$ process is a significant part of the cross section. Since the Higgs is produced by color singlet $W$ bosons, the central region in rapidity should have less jet activity in it for Higgs events than for the background, particularly for that from $t \bar{t}$. At low luminosity, requiring that the events have no additional jets (apart from the ones that make up the $\mathrm{W}$ candidate) with $E_{T}>20 \mathrm{GeV}$ and $|\eta|<2$ loses approximately $35 \%$ of the signal and reduces the background from $W+$ jets $(t \bar{t})$ by a factor of 2.5 (12).

Forward jet tagging was investigated in ATLAS as follows. Clusters of energy of size $\Delta R=0.5$ were found in the region $2<|\eta|<5$. Events from the pile up of minimum bias events have jets in these regions so the threshold on $E_{T}$ of the jet must be set high enough so that these jets do not generate tags in the background. If the individual calorimeter cells are required to have $E_{T}>3 \mathrm{GeV}$, then there is there is a $4.6 \%(0.07 \%)$ probability that the pile up at high luminosity will contribute a single (double) tag to an event that would otherwise not have one for tagging jets with $E_{T}>15 \mathrm{GeV}$ and $E>600 \mathrm{GeV}$. The requirement of a double tag is then applied to the signal from a Higgs boson of mass $1 \mathrm{TeV}$ and the various backgrounds. The pile up contributions are included and the event rates for a luminosity of $30 \mathrm{fb}^{-1}$ shown in table f. The effect of a change in the tagging criteria can be seen in Fig. 12 which shows the variation of the shape in the background. The $Z Z$ final state is cleaner as there is no $t \bar{t}$ background but the event rates are much smaller.

A separate study was performed by the CMS group [2, 48]. Here two tagging jets with $|\eta|>2.4, E_{T}>10 \mathrm{GeV}$ and $E>400 \mathrm{GeV}$ are required. Two central jets are required with in invariant mass within $15 \mathrm{GeV}$ of the $W$ or $Z$ mass. For the $Z Z$ case, the $Z$ is reconstructed from $e$ or $\mu$ pairs with invariant mass within $10 \mathrm{GeV}$ of the $\mathrm{Z}$ mass; each lepton has $p_{T}>50 \mathrm{GeV}$ and the pair has $p_{T}>150 \mathrm{GeV}$. For the $W W$ case, at least $150 \mathrm{GeV}$ of missing $E_{T}$ is needed and the charged lepton from the $W$ has $p_{T}>150 \mathrm{GeV}$.

It can be seen from Table that it will be possible to extract a signal although there are large uncertainties on the estimated background. However, other kinematic quantities may be used to further discriminate between the signal and the background. The $Z Z$ final state is cleaner as there is no $t \bar{t}$ background but the event rates are much smaller.

\section{Measurements of Higgs properties}

A Standard Model Higgs should have a mass between about $113.5 \mathrm{GeV}$ and $212 \mathrm{GeV}$ [20]. Over this mass range the branching ratios and other properties of the Higgs vary rapidly, but they are precisely predicted in terms of the mass. In the $\gamma \gamma$ and four-lepton channels, the mass resolution is typically $1 \%$, and the energy scale can be calibrated to better than $0.1 \%$ using $Z \rightarrow e e$ and $Z \rightarrow \mu \mu$ events. Fig. 13 shows that the mass can be measured to $\sim 0.1 \%$ for all favored masses [35].

Higgs branching ratios cannot be determined directly at the LHC, but it is possible to infer combinations of couplings from

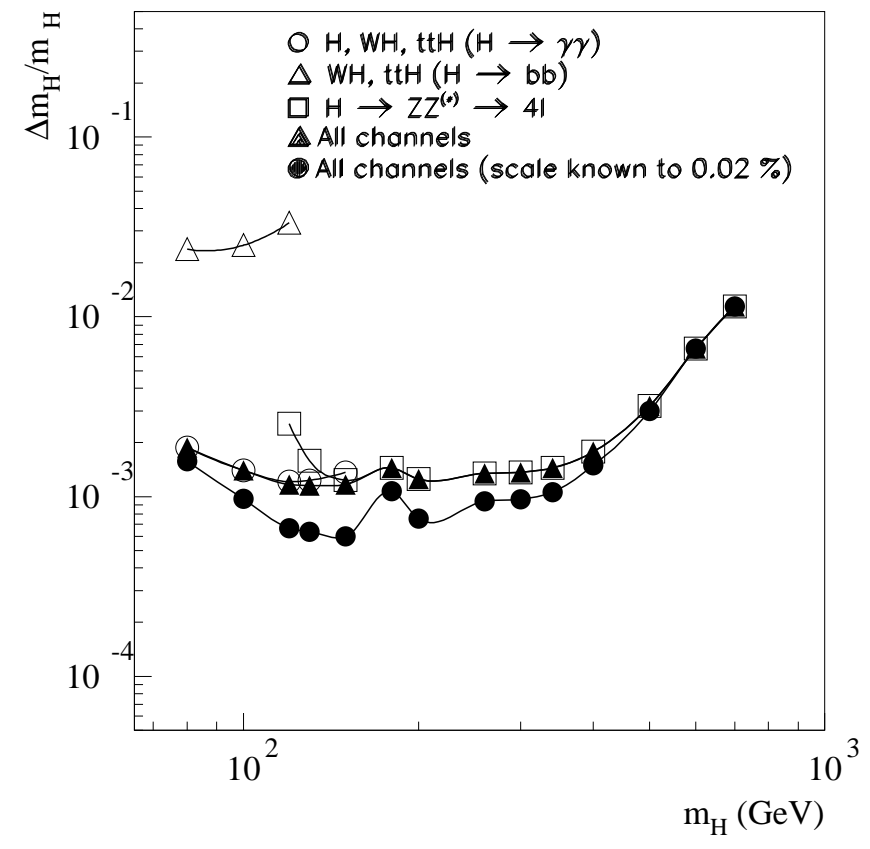

Figure 13: Expected ultimate errors on the Higgs mass in ATLAS. From Ref. [35].

measured rates. The dominant Higgs production mode is $g g \rightarrow$ $H$, so measurements of the inclusive $H \rightarrow \gamma \gamma$ and $H \rightarrow Z Z^{*}$ cross sections as discussed above can be used to determine the product of the $H g g$ and the $H \gamma \gamma$ or $H Z Z$ couplings. The $H g g$ coupling in turn is related to the $H t \bar{t}$ one.

More information can be obtained by making use of the $W W$ fusion process. About $10 \%$ of the total cross section in this mass range comes from $q q \rightarrow q q H$ via the exchange of two virtual $W$ bosons:

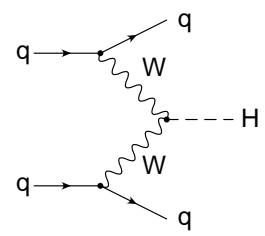

The probability that a virtual $W$ is radiated carrying a fraction $x$ of the momentum of the incoming quark behaves like $d x / x$ at small $x$, so the outgoing quarks typically have large momentum. Thus, the $W W$ fusion process can be identified by requiring high-energy jets with $p_{T} \sim M_{W}$ in the forward calorimeters and no additional QCD radiation in the central region. These requirements greatly reduce the QCD backgrounds. Exploitation of this process requires a detailed understanding of the forward jet tagging. Complete simulations of these have not yet been completed.

The estimated statistical errors on the cross sections for a number of Higgs production and decay channels are shown in Fig. 14. These have been calculated by applying selection criteria developed for various Higgs searches separately for ATLAS and CMS, calculating the errors $\Delta \sigma / \sigma=\sqrt{S+B} / S$, and combining the results [49]. The $W W \rightarrow H \rightarrow \tau \tau$ channel is reconstructed using the fact that $W W$ fusion provides a 


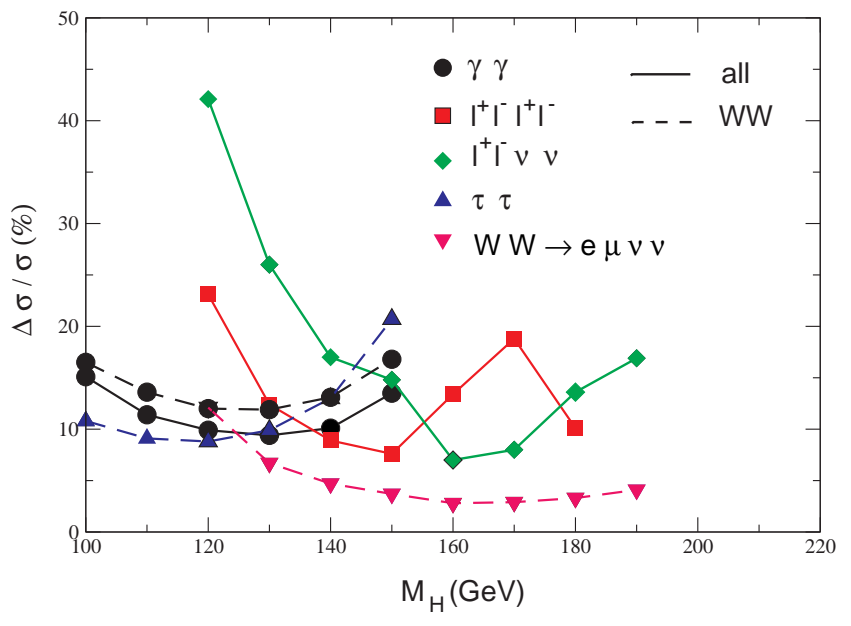

Figure 14: Estimated statistical errors on the cross sections for inclusive Higgs production and production via $W W$ fusion with decays into various modes for $100 \mathrm{fb}^{-1}\left(30 \mathrm{fb}^{-1}\right.$ for the $\ell^{+} \ell^{-} \nu \bar{\nu}$ mode). Based on Ref. [49].

transverse boost to the Higgs, so that one can project the $\mathbb{E}_{T}$ along the two measured $\tau$ directions and reconstruct the mass, as discussed in connection with the search for $A \rightarrow \tau \tau$ below. Note that for each Higgs mass there are several channels that can be measured with statistical errors between $5 \%$ and $20 \%$.

It is of course necessary to correct these measurements for acceptance. For a process like $g g \rightarrow H \rightarrow \gamma \gamma$ or $g g \rightarrow H \rightarrow$ $\ell^{+} \ell^{-} \ell^{+} \ell^{-}$this is relatively straightforward. The signal is a narrow bump on a smooth background, and the losses from geometrical acceptance, isolation cuts, etc., are relatively small and understood. The acceptance and background corrections for the forward jet tags needed to select $W W$ fusion are more difficult to estimate. Ultimately it will be necessary to vary the cuts and compare the results with both Monte Carlo event generators and matrix element calculations. The $W W \rightarrow H \rightarrow \tau \tau$ channel also has difficult corrections related to the $\tau$ identification and measurement. After the corrected cross sections are obtained, they must be compared with perturbative QCD calculations of the cross sections to determine the relevant combination of couplings. These calculations are known to NLO in all cases and have recently been calculated to NNLO for the $g g \rightarrow H$ processes.

Studies of this program of measurements are actively underway in both ATLAS and CMS. Reliable estimates of the expected errors are not yet available, but it seems plausible that measurements for several channels will be possible with errors in the 10\%-20\% range. This will provide a significant amount of information on the couplings of the Higgs.

\section{Summary of Standard Model Higgs}

The LHC at full luminosity will be able to probe the entire range of Higgs masses from the lower limit set by LEP up to the value where it is no longer sensible to speak of an elementary Higgs boson. The search mainly relies only on final states that one is confident will be effective: $\gamma \gamma, 4 \ell$ and $2 \ell \nu \bar{\nu}$. Additional final states that afford an excellent chance of having a signal will be exploited to support these: $b \bar{b}$ with an associated lepton tag at low mass, and $\ell \nu+j e t s$ and $\ell \ell+j e t s$ at high mass. The failure to find a Higgs boson over this range would therefore enable the Standard Model to be ruled out. The Higgs sector then either consists of non-standard Higgs bosons or the electroweak symmetry breaking occurs via some strongly coupled process that will manifest itself in the study of $W W$ scattering. The next subsection is devoted to an example of the former type.

\section{B. SUSY Higgs}

As stated above the minimal supersymmetry model (MSSM) has three neutral and one charged Higgs bosons; $h, H, A$ and $H^{ \pm}$. These arise because supersymmetric models, unlike the Standard Model, need different Higgs bosons to generate masses for the up and down type quarks. In the Standard Model one parameter, the Higgs mass, is sufficient to fully fix its properties. In the MSSM, two parameters are needed. These can be taken to be the mass of $A$ and the ratio $(\tan \beta)$ of the vacuum expectation values of the Higgs fields that couple to up-type and down-type quarks. If $\tan \beta$ is $O(1)$, then coupling of the top quark to Higgs bosons $\left(\lambda_{t}\right)$ is much larger than that of bottom quarks $\left(\lambda_{b}\right)$ as is the case in the Standard Model.

None of these Higgs bosons has been observed, so we need consider only the regions of parameter space not yet excluded. At tree level the masses of $h$ and $H$ are given in terms of the mass of $A$ and $\tan \beta$. The charged Higgs boson $H^{ \pm}$is heavier than $A\left(M_{H^{ \pm}}^{2} \sim M_{A}^{2}+M_{W}^{2}\right)$. The $H$ is heavier than the $A$, while the $A$ and $H$ are almost degenerate at large values of $M_{A}$, The mass of the lightest boson, $h$, increases with the mass of $A$ and reaches a plateau for $A$ heavier than about $200 \mathrm{GeV}$. The actual values depend on the masses of the other particles in the theory particularly the top quark [50]. There is also a dependence (via radiative corrections) on the unknown masses and other parameters of the other supersymmetric particles. This dependence is small if these particles are heavy, so it is conventional to assume that this is the case.

In the limit of large $A$ mass, the couplings of the Higgs bosons are easy to describe. The couplings of $h$ become like those of the Standard Model Higgs boson. The couplings of $A$ and $H$ to charge $1 / 3$ quarks and leptons are enhanced at large $\tan \beta$ relative to those of a Standard Model Higgs boson of the same mass. However, $A$ does not couple to gauge boson pairs at lowest order and the coupling of $H$ to them is suppressed at large $\tan \beta$ and large $M_{A}$. The decay modes used above in the case of the Standard Model Higgs boson can also be exploited in the SUSY Higgs case. $h$ can be searched for in the final state $\gamma \gamma$, as the branching ratio approaches that for the Standard Model Higgs in the large $M_{A}$ (decoupling) limit.

The decay $A \rightarrow \gamma \gamma$ can also be exploited. This has the advantage that, because $A \rightarrow Z Z$ and $A \rightarrow W W$ do not occur, the branching ratio is large enough for the signal to be usable for values of $M_{A}$ less than $2 m_{t}$ [51]. The decay $H \rightarrow Z Z^{*}$ can be exploited, but at large values of $M_{H}$ the decay $H \rightarrow Z Z$, which provides a very clear signal for the Standard Model Higgs, is useless owing to its very small branching ratio, The channel 


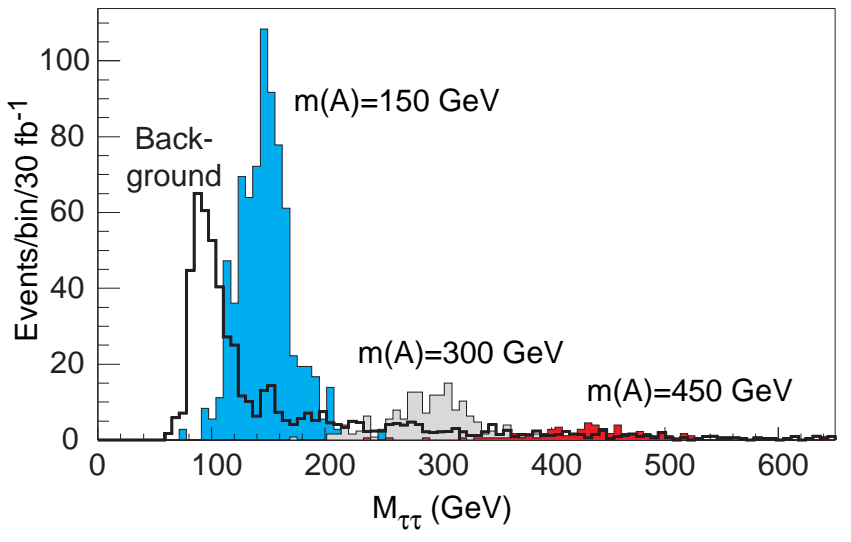

Figure 15: Reconstructed $\tau \tau$ invariant mass after projecting measured $\mathbb{E}_{T}$ along the observed $\tau$ directions. ATLAS from Ref. [54].

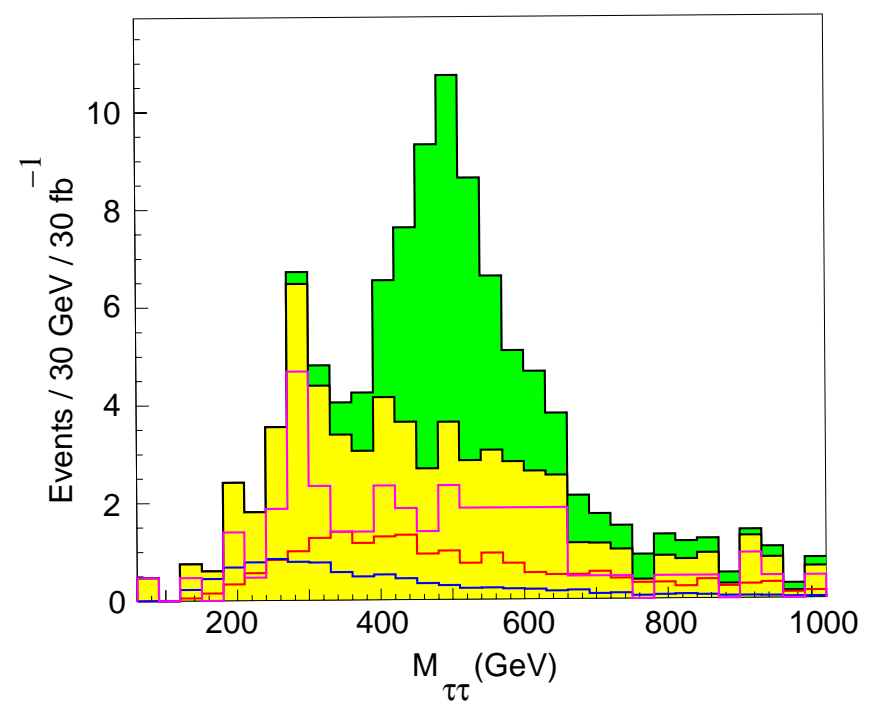

Figure 16: Reconstructed $\tau \tau$ invariant mass for $M_{H}=$ $500 \mathrm{GeV}$ and $\tan \beta=25$ from a CMS simulation. Ref. [55].

$t \bar{t} h \rightarrow t \bar{t} b \bar{b}$ can also be exploited.

In addition to these decay channels, several other possibilities open up due to the larger number of Higgs bosons and possibly enhanced branching ratios. The most important of these are the decays of $H$ and $A$ to $\tau^{+} \tau^{-}$and $\mu^{+} \mu^{-}, H \rightarrow h h, A \rightarrow Z h$ and $A \rightarrow t \bar{t}$.

It is important to remark that the effect of supersymmetric particles is ignored in this section. That is, the possible decays of Higgs bosons to supersymmetric particles are not considered and supersymmetric particles have been assumed to be heavier than $1 \mathrm{TeV}$, so that their effects on branching ratios and production rates via radiative corrections are ignored. Some effects of these decays have been studied [35]; the section below on supersymmetry discusses the case where Higgs bosons can be produced in the decays of supersymmetric particles.

\section{1. $H / A \rightarrow \tau \tau$}

In the MSSM, the $H \rightarrow \tau^{+} \tau^{-}$and $A \rightarrow \tau^{+} \tau^{-}$rates are strongly enhanced over the Standard Model if $\tan \beta$ is large, resulting in the possibility of observation over a large region of parameter space. The $\tau^{+} \tau^{-}$signature can be searched for either in a lepton+hadron final state, or an $e+\mu$ final state. As there are always neutrinos to contend with, mass reconstruction is difficult, and $\mathbb{E}_{T}$ resolution is critical. In ATLAS, at high luminosity this resolution is

$$
\sigma\left(\mathbb{E}_{T, x}\right)=\sigma\left(\mathbb{E}_{T, y}\right)=0.46 \sqrt{\sum E_{T}}
$$

where all energies are measured in $\mathrm{GeV}$. Irreducible backgrounds arise from Drell-Yan tau pair production, $t \bar{t}$ and $b \bar{b}$ decays to $\tau \tau$. Both CMS [52] and ATLAS [53] have studied $\tau^{+} \tau^{-}$ final states using full simulation.

For the lepton+hadron final state, there are additional reducible backgrounds from events with one hard lepton plus a jet that is misidentified as a tau. In the CMS and ATLAS studies, events were required to have one isolated lepton with $p_{T}>15-40 \mathrm{GeV}$ depending on $m_{A}(\mathrm{CMS})$ or $p_{T}>24 \mathrm{GeV}$ (ATLAS) within $|\eta|<2.0(2.4)$ and one tau-jet candidate within $|\eta|<2.0(2.5)$.

ATLAS required that the tau jet have $E_{T}>40 \mathrm{GeV}$, that the radius of the jet computed only from the EM cells be less than 0.07 ; that less than $10 \%$ of its transverse energy be between $R=0.1$ and $R=0.2$ of its axis; and again, that exactly one charged track with $p_{T}>2 \mathrm{GeV}$ point to the cluster. The CMS and ATLAS selections are about $40 \%(26 \%)$ efficient for taus, while accepting only $1 / 100(1 / 400)$ of ordinary light quark and gluon jets.

CMS vetoed events having other jets with $E_{T}>25 \mathrm{GeV}$ within $|\eta|<2.4$ (this reduces the $t \bar{t}$ background); while ATLAS used cuts on $\mathbb{E}_{T}$, the transverse mass formed from the lepton and $\mathbb{E}_{T}$, and the azimuthal angle between the lepton and the tau-jet. The mass of the Higgs may be reconstructed by assuming the neutrino directions to be parallel to those of the lepton and the tau-jet. Resolutions of 12 and $14 \mathrm{GeV}$ (Gaussian part) are obtained by ATLAS and CMS for $m_{A}=100 \mathrm{GeV}$. The reconstructed Higgs peaks as simulated by ATLAS for several masses are shown in Fig. 15; a CMS simulation is shown in Fig. 16 .

Both ATLAS and CMS find the sensitivity in the $e+\mu$ final state to be less than for the lepton+hadron final state, owing to its smaller rate and less favorable decay kinematics.

Taking the lepton + hadron and $e+\mu$ modes together, for the sum of $H$ and $A$ decays, both ATLAS and CMS find that the large region of parameter space corresponding to $\tan \beta \gtrsim 6$ at $m_{A}=125 \mathrm{GeV}$ rising to $\tan \beta \gtrsim 30$ at $m_{A}=500 \mathrm{GeV}$ may be excluded at the $5 \sigma$ confidence level with $30 \mathrm{fb}^{-1}$. ATLAS also finds some sensitivity to $\tan \beta \lesssim 2$ for $125<m_{A}<350 \mathrm{GeV}$ at very high integrated luminosities $\left(300 \mathrm{fb}^{-1}\right)$.

$$
\text { 2. } H / A \rightarrow \mu \mu
$$

The branching ratio for $H$ (or $A$ ) to $\mu^{+} \mu^{-}$is smaller than that to $\tau^{+} \tau^{-}$by a factor of $\left(m_{\mu} / m_{\tau}\right)^{2}$. The better resolution available in this channel compensates to some extent for this and the 


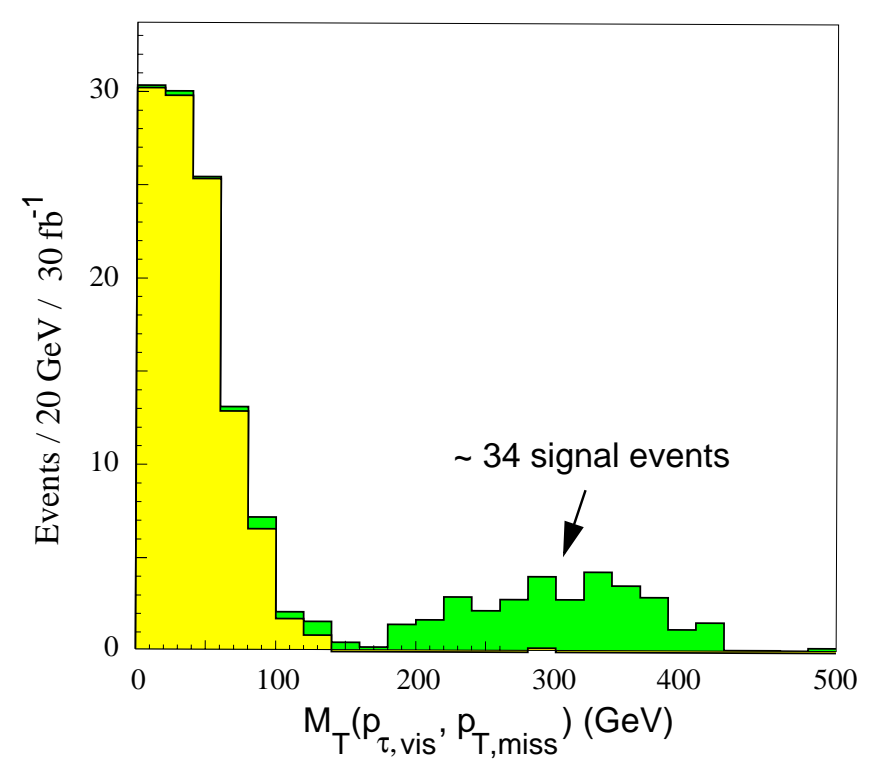

Figure 17: The transverse mass distribution of signal and backgrounds for a charged Higgs search using $30 \mathrm{fb}^{-1}$. The couplings are determined in the MSSM with $M_{H^{+}}=409 \mathrm{GeV}$ and $\tan \beta=40$. From Ref. [56].

$\mu^{+} \mu^{-}$mode can be useful for large values of $\tan \beta$. A signal of less statistical significance than that in the $\tau^{+} \tau^{-}$could be used to confirm the discovery and make a more precise measurement of the mass and production cross section. The ATLAS analysis [35] requires two isolated muons with $p_{T}>20 \mathrm{GeV}$ and $|\eta|<2.5$. The background from $t \bar{t}$ events is rejected by requiring $\mathbb{E}_{T}<20$ (40) GeV at low (high) luminosity. A jet veto could be employed to reduce this background further, but this is ineffective at reducing the remaining dominant background for $\mu^{+} \mu^{-}$pairs from the Drell-Yan process. A cut on the transverse momentum of the muon pair, requiring it to be less than 100 $\mathrm{GeV}$, reduces the $t \bar{t}$ background further. The remaining background is very large within $\pm 15 \mathrm{GeV}$ of the $\mathrm{Z}$ mass. Above this region the signal appears as a narrow peak in the $\mu^{+} \mu^{-}$mass spectrum. In this region the signal will be statistically significant if $\tan \beta$ is large enough but it appears as a shoulder on the edge of a steeply falling distribution which may make it more difficult to extract a signal.

The significance of the signal in this channel is determined by the $\mu^{+} \mu^{-}$mass resolution and the intrinsic width of the Higgs resonance. The mass resolution in ATLAS is approximately $0.02 m_{A}$ and is $0.013 m_{A}$ in CMS [58]. At large $\tan \beta$, the masses of $A$ and $H$ are almost degenerate and they cannot be resolved from each other. The natural width of $A$ is proportional to $\tan ^{2} \beta$ and is approximately $3 \mathrm{GeV}$ for $\tan \beta=30$ and $M_{A}=150 \mathrm{GeV}$. The mode will provide a $5 \sigma$ signal for a region in the $M_{A}-\tan \beta$ plane covering $M_{A}>110 \mathrm{GeV}$ and $\tan \beta>15$ for an integrated luminosity of $10^{5} \mathrm{fb}^{-1}$.

\footnotetext{
${ }^{2}$ The CMS event rates appear larger than the ATLAS ones. CMS added the $A$ and $H$ rates whereas the ATLAS numbers correspond to the $A$ alone.
}

\section{3. $A \rightarrow \gamma \gamma$}

Gluon fusion $(g g \rightarrow A)$ via top and bottom quark triangle loop diagrams is the dominant production process if $\tan \beta \lesssim 4$; while for large $\tan \beta$ ( $\gtrsim 7$ ) b-quark fusion dominates. For $\tan \beta \approx 1$ and $170 \mathrm{GeV}<m_{A}<2 m_{t}$ the branching fraction of $A \rightarrow \gamma \gamma$ is between $5 \times 10^{-4}$ and $2 \times 10^{-3}$. The backgrounds considered are QCD photon production, both the irreducible two-photon backgrounds ( $q \bar{q} \rightarrow \gamma \gamma$ and $g g \rightarrow \gamma \gamma$ ) and the reducible backgrounds with one real photon $(q \bar{q} \rightarrow g \gamma$, $q g \rightarrow q \gamma$, and $g g \rightarrow g \gamma)$. In the ATLAS study [35], both photons were required to have $|\eta|<2.5$, one with $P_{T}>125 \mathrm{GeV}$ and the other with $p_{T}>25 \mathrm{GeV}$. Both photons are required to be isolated. The signal is effective at small values of $\tan \beta$ for $2 m_{t}<M_{A}<200 \mathrm{GeV}$.

\section{Search for Charged Higgs}

In extensions of the Standard Model with charged Higgs bosons $H^{ \pm}$, such as in the MSSM, the decay $t \rightarrow b H^{ \pm}$may compete with the standard $t \rightarrow b W^{ \pm}$if kinematically allowed. The $H^{ \pm}$decays to $\tau \nu$ or $c \bar{s}$ depending on the value of $\tan \beta$. Over most of the range $1<\tan \beta<50$, the decay mode $H^{ \pm} \rightarrow \tau \nu$ dominates. The signal for $H^{ \pm}$production is thus an excess of taus produced in $t \bar{t}$ events.

Both ATLAS [92] and CMS [93] have investigated the sensitivity to this excess. Top events with at least one isolated high$p_{T}$ lepton are selected, and the number having an additional tau compared with the number having an additional $e$ or $\mu$. Both studies used $b$-tagging to reduce the backgrounds to top production. Taus were identified in a way very similar to that described earlier (in the section on $A, H \rightarrow \tau \tau$ searches). The uncertainty in the tau excess is estimated to be $\pm 3 \%$, dominated by systematics. For an integrated luminosity of $10 \mathrm{fb}^{-1}$, both ATLAS and CMS conclude that over most of the $\tan \beta$ range, a signal can be observed at the $5 \sigma$ level for $m_{H^{ \pm}}<130 \mathrm{GeV}$, which corresponds to the region $m_{A} \lesssim 120 \mathrm{GeV}$ in the $m_{A}, \tan \beta$ plane.

If a charged Higgs boson has larger mass than the top then it cannot be produced in the decay of a top quark. In this case the relevant production mechanism is the $g b \rightarrow H^{-} t$ [56, 57]. The signal can be searched for via the decay $H^{+} \rightarrow \tau \nu$. The tau is searched for via its hadronic decay which gives rise to isolated single hadrons (either $\pi$ or $K$ ). This track is required to have $p_{T}>100 \mathrm{GeV}$. Events are then required to have a single tagged $b$-jet and two other jets whose masses are consistent with the decay $t \rightarrow W b \rightarrow q q b$ and $\mathbb{E}_{T}>100 \mathrm{GeV}$. Events with two tagged $b$-jets are vetoed. A transverse mass is then formed between the reconstructed single hadron and the $\mathbb{E}_{T}$. The distribution of this transverse mass is shown in Fig. 17 for a charged Higgs mass of $500 \mathrm{GeV}$. The Standard Model background is small. Note that the peak is below the mass of the charged Higgs. This is due to the partial cancellation of missing $E_{T}$ from the two neutrinos in the decay chain $H^{+} \rightarrow \tau \nu \rightarrow \pi \nu \nu$.

\section{Other possible Higgs signatures}

Observation of the channel $H \rightarrow h h$ would be particularly interesting as information about two different Higgs bosons and their coupling could be obtained. The dominant decay here is 


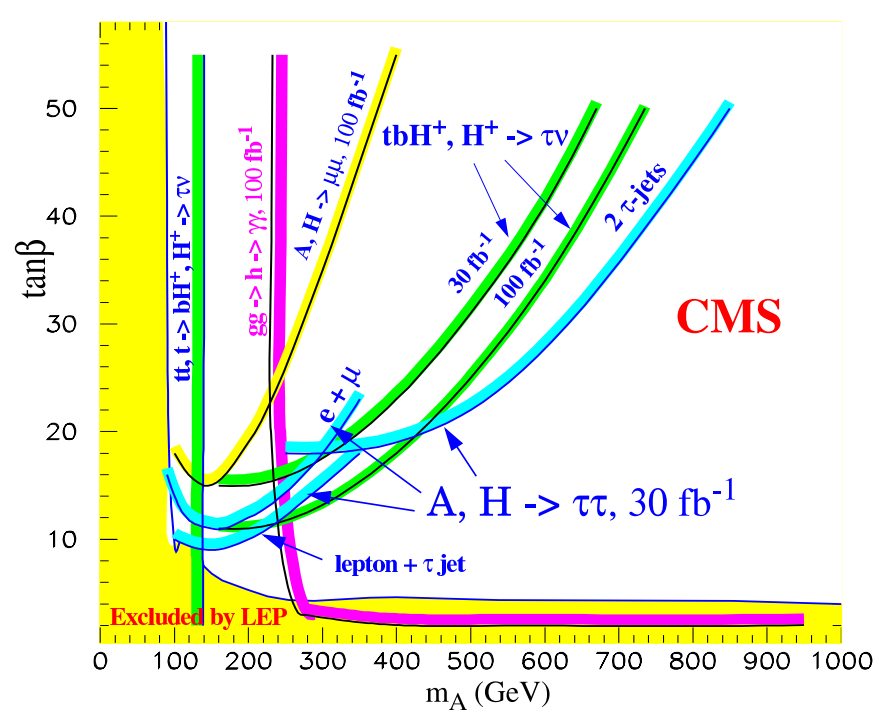

Figure 18: $5 \sigma$ discovery contours for the various processes used to search for Higgs bosons in the MSSM. This plot assumes no stop mixing, maximizing the reach of LEP. From Ref. [59].

to the final state $b \bar{b} b \bar{b}$. However it is not clear how this mode could be triggered efficiently. If a trigger could be constructed - perhaps using soft muons in jets - then the process is sensitive for $\tan \beta<3$ and $250<M_{A}<2 m_{t}$. The channel $H \rightarrow h h \rightarrow b \bar{b} \tau^{+} \tau^{-}$can be triggered and is being studied. The LEP Higgs limits exclude most of the accessible region in the MSSM, but these channels might be observable in more general models.

The decay channel $H \rightarrow h h \rightarrow \gamma \gamma b \bar{b}$ is triggerable and was studied [35]. Events were required to have a pair of isolated photons with $|\eta|<2.5$ and $p_{T}>20 \mathrm{GeV}$ and two jets with $p_{T}>15(30) \mathrm{GeV}$ and $|\eta|<2.5$ at low (high) luminosity. One of the jets was required to be tagged as a $b$-jet. No other jets with $P_{T}>30 \mathrm{GeV}$ were allowed in the region $|\eta|<2.5$. The dominant background arises from $\gamma \gamma$ production in association with light quark jets and is approximately 10 times larger than the $\gamma \gamma b \bar{b}$ background. Event rates are very low, for $M_{H} \sim 250 \mathrm{GeV}$ and $m_{h}=100 \mathrm{GeV}$ there are about 15 signal events for 200 $\mathrm{fb}^{-1}$ of integrated luminosity. However the very small background ( $\sim 2$ events for $200 \mathrm{fb}^{-1}$ ) and the sharp peak in the $\gamma \gamma$ mass distribution should provide convincing evidence of a signal.

For large masses, the $A$ and $H$ decay almost exclusively to $t \bar{t}$. The background in this channel arises from QCD $t \bar{t}$ production. While this background is very large, a statistically significant signal can be extracted provided that the background can be calibrated [35]. The signal is searched for in the final state $W W b \bar{b}$ where one $\mathrm{W}$ decays leptonically. For an integrated luminosity of $30 \mathrm{fb}^{-1}$ there are about 2000 events for $M_{A} \sim 400 \mathrm{GeV}$ after cuts requiring an isolated lepton (which provides the trigger) and a pair of tagged $b$-quark jets. The $t \bar{t}$ mass resolution is of order $15 \mathrm{GeV}$ resulting in approximately 40000 background events. The rate for $t \bar{t}$ production is well predicted by perturbative QCD, so it may well be possible to establish an event excess but extraction of a mass for $A$ will be very difficult as there is no observable mass peak. The mode is most likely to be useful as confirmation of a signal seen elsewhere.

The decay $A \rightarrow Z h$ offers another channel where two Higgs bosons might be observed simultaneously. The leptonic decay of the $Z$ can be used as a trigger. The CMS study requires a pair of electrons (muons) with $p_{T}>20$ (5) $\mathrm{GeV}$ which have an invariant mass within $6 \mathrm{GeV}$ of the $Z$ mass and a pair of jets with $p_{T}>40 \mathrm{GeV}$. One or two $b$-tags are required with an assumed efficiency of $40 \%$ and a rejection of 50 against light quark jets. The background is dominated by $t \bar{t}$ events. The signal to background ratio is quite good for moderate $M_{A}$ and small $\tan \beta$, but this region is excluded in the MSSM by the LEP Higgs limits.

The positive conclusion of this study is confirmed in [35] where several values of $M_{A}$ and $m_{h}$ were simulated and it was concluded that a $5 \sigma$ signal is observable for an integrated luminosity of $30 \mathrm{fb}^{-1}$ for $\tan \beta<2$ and $150<M_{A}<350$. This study included the background from $Z b \bar{b}$ events which dominate over the $t \bar{t}$ background at smaller values of $m_{A}$.

\section{Summary of Supersymmetric Higgs}

One is confident that the following modes will be effective in searching for the MSSM Higgs bosons: $A / H \rightarrow \tau^{+} \tau^{-}$, $A / H \rightarrow \mu^{+} \mu^{-}, H^{+} \rightarrow \tau \nu, H \rightarrow Z Z^{*} \rightarrow 4 \ell, h \rightarrow \gamma \gamma$, $A \rightarrow Z h \rightarrow \ell \ell b \bar{b}, H \rightarrow h h \rightarrow b \bar{b} \gamma \gamma$ and $t \rightarrow b H^{+}(\rightarrow \tau \nu)$ (discussed in the section on the top quark). In addition, the modes $A / H \rightarrow t \bar{t}$ and $h \rightarrow b \bar{b}$ produced in association with a $W$ or $t \bar{t}$ may provide valuable information. The former set of modes are sufficient for either experiment to exclude the entire $\tan \beta-M_{A}$ plane at $95 \%$ confidence with $100 \mathrm{fb}^{-1}$.

Ensuring a $5 \sigma$ discovery over the entire $\tan \beta-M_{A}$ plane requires more luminosity. Figs. 18 and 19 show what can be achieved. The entire plane is covered using the modes where one has great confidence. Over a significant fraction of the parameter space at least two distinct modes will be visible. Over a significant fraction of the phase space beyond the LEP limit, $h \rightarrow \gamma \gamma, H^{+} \rightarrow \tau \nu_{\tau}$ and $H / A \rightarrow \tau \tau(H / A \rightarrow \mu \mu)$ will be measured. The decay of other supersymmetric particles will provide additional sources of $h$. Over a significant fraction of SUSY parameter space, there is a substantial branching fraction for sparticles to decay to $h$. The rate is then such that decay $h \rightarrow b \bar{b}$ becomes clearly observable above background and this channel is the one where $h$ is observed first at LHC (see below).

\section{SUPERSYMMETRY}

If SUSY is relevant to electroweak symmetry breaking, then the arguments summarized above suggest that the gluino and squark masses are less than $\mathcal{O}(1 \mathrm{TeV})$, although squarks might be heavier. As many supersymmetric particles can be produced simultaneously at the LHC, a model that has a consistent set of masses and branching ratios must be used for simulation. Analysis of the simulated events is performed without reference to the underlying model. The SUGRA model [60] assumes that gravity is responsible for the mediation of supersymmetry breaking and provides a natural candidate for cold dark matter. 


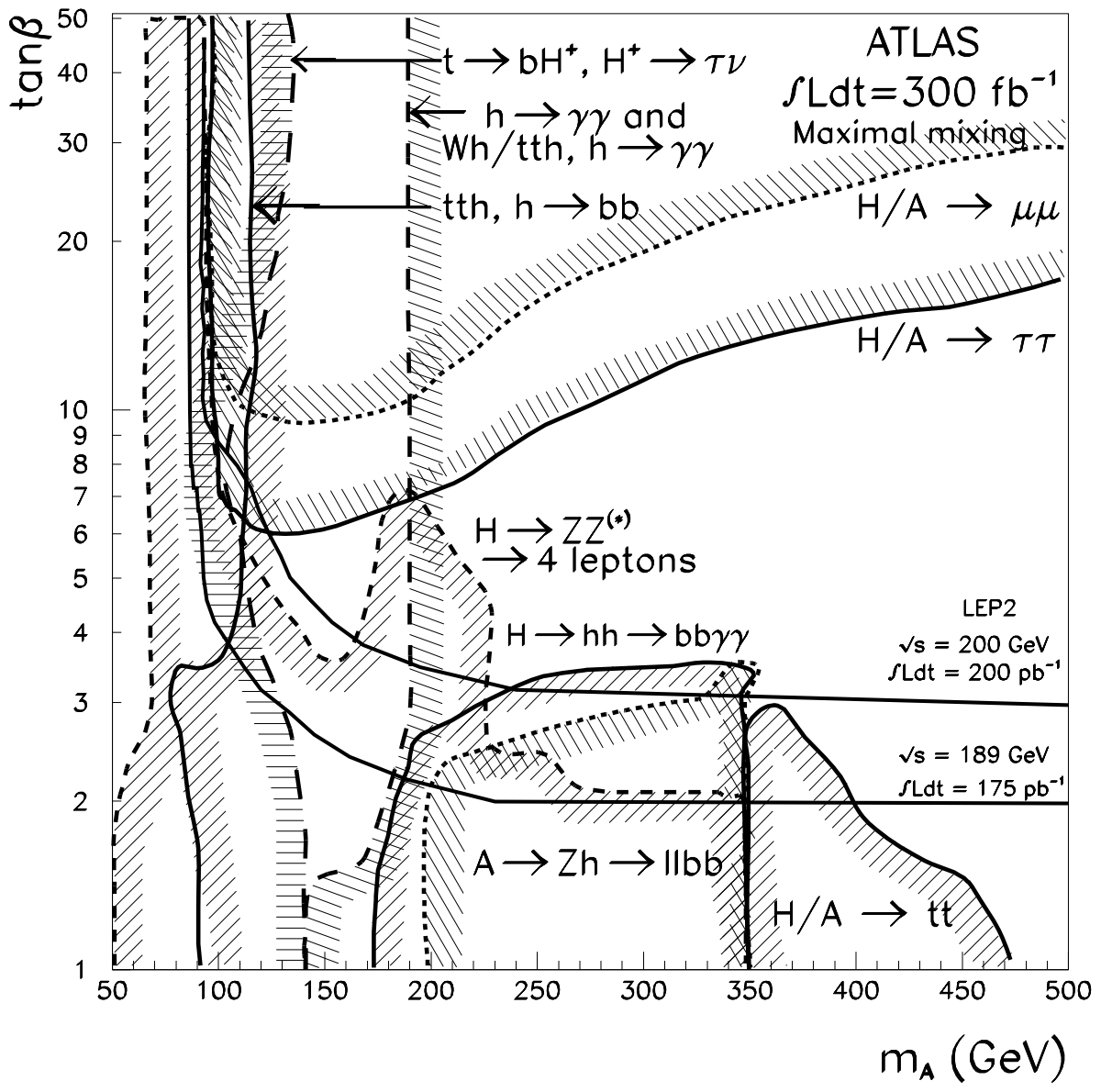

Figure 19: $5 \sigma$ discovery contours for the various processes used to search for Higgs bosons in the MSSM. This plot assumes maximal stop mixing, minimizing the reach of LEP. From Ref. [35].

The GMSB model 61 assumes that Standard Model gauge interactions are responsible for the mediation and explains why flavor changing neutral current effects are small. Anomaly mediation is always present [62]; the AMSB model assumes that it is dominant.

Gluinos and squarks usually dominate the LHC SUSY production cross section, which is of order $10 \mathrm{pb}$ for masses around $1 \mathrm{TeV}$. Since these are strongly produced, it is easy to separate SUSY from Standard Model backgrounds provided only that the SUSY decays are distinctive. In the minimal SUGRA model these decays produce $\mathbb{E}_{T}$ from the missing $\tilde{\chi}_{1}^{0}$ 's plus multiple jets and varying numbers of leptons from the intermediate gauginos. Fig. 20 shows the $5 \sigma$ reach in this model at the LHC for $100 \mathrm{fb}^{-1}$ 63 The reach is not very sensitive to the fixed parameters $(A$ and $\tan \beta$ ). It is considerably more than the expected mass range even for $10 \mathrm{fb}^{-1}$ as can be seen from Fig. 21 which shows how the accessible mass range depends upon integrated luminosity. This plot also shows the parameter range over which the model provides a suitable dark matter candidate.

A typical example of the signatures whose reach is shown in Figure 20, is the distribution of the "effective mass"

$$
M_{\mathrm{eff}}=\mathbb{E}_{T}+\sum_{i=1}^{4} p_{T, i}
$$

computed from the missing energy and the four hardest jets. This is shown in Fig. 22 after multijet and $\mathbb{E}_{T}$ cuts for a SUGRA point 64] with gluino and squark masses of about $700 \mathrm{GeV}$.

While the reach in Fig. 20 has been calculated for a specific SUSY model, the multiple jet plus $\mathbb{E}_{T}$ signature is generic in most $R$ parity conserving models. GMSB models can give additional photons or leptons or long-lived sleptons with high $p_{T}$ but $\beta<1$, making the search easier. $R$-parity violating models with leptonic $\tilde{\chi}_{1}^{0}$ decays also give extra leptons and very likely violate $e-\mu$ universality. $R$-parity violating models with $\tilde{\chi}_{1}^{0} \rightarrow q q q$ give signals at the LHC with very large jet multiplicity, for which the Standard Model background is not well known. For such models, it may be necessary to rely on leptons produced in the cascade decay of the gluinos and squarks. In all cases, SUSY can be discovered at the LHC if the masses are in the expected range, and simple kinematic distributions can be used to estimate the approximate mass scale [35].

\section{A. SUGRA Measurements}

The main problem at the LHC is not to observe a SUSY signal that deviates from the Standard Model but to separate the many different channels produced by all the SUSY cascade decays from the produced squarks and gluinos. In SUGRA and many 


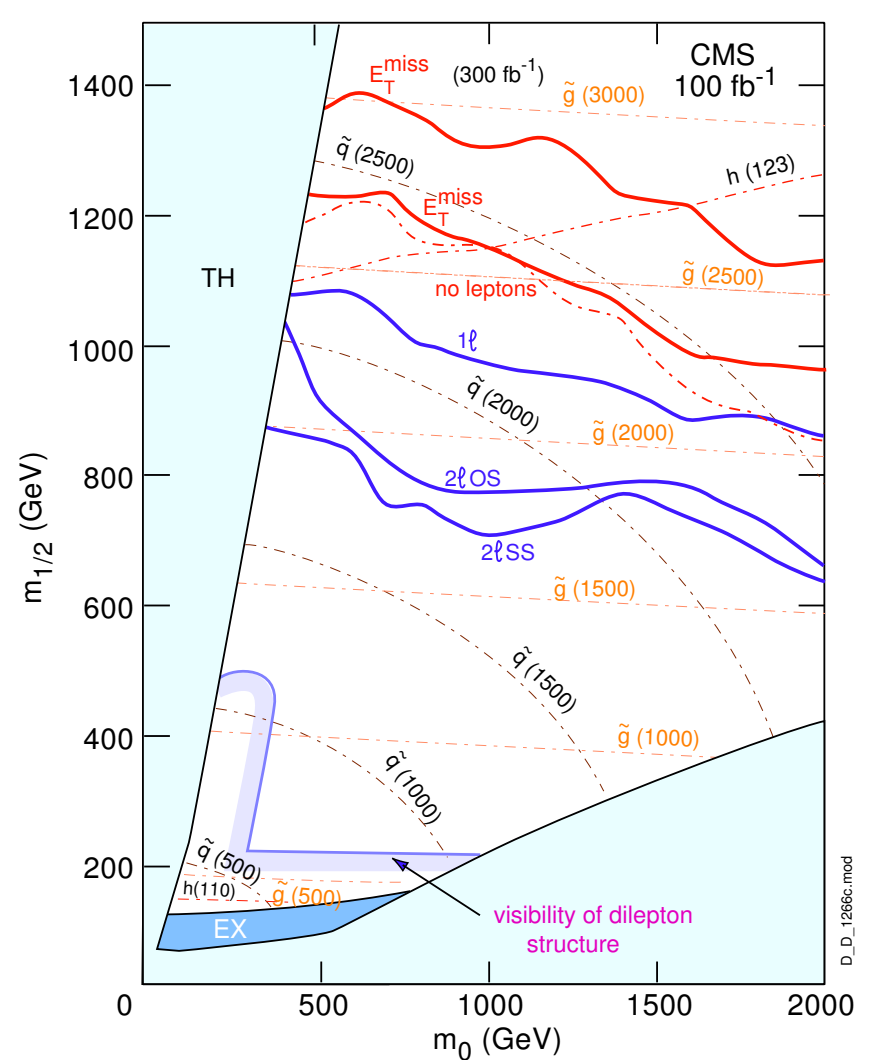

Figure 20: Plot of $5 \sigma$ reach in minimal SUGRA model for $\tan \beta=35$ and $\mu=+$ at LHC with $100 \mathrm{fb}^{-1}$ for $\mathbb{E}_{T}$ inclu-

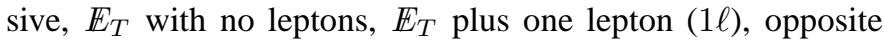

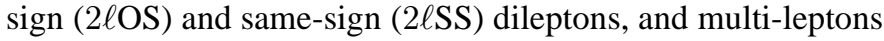
$(3 \ell, 4 \ell)$. The region where a dilepton edge is visible is indicated. From Ref. [63].

other models, the decay products of SUSY particles always contain an invisible $\tilde{\chi}_{1}^{0}$, so no masses can be reconstructed directly. One promising approach is to try to identify particular decay chains and to measure kinematic endpoints for combinations of visible particles in these [66]. For example, the $\ell^{+} \ell^{-}$mass distribution from $\tilde{\chi}_{2}^{0} \rightarrow \tilde{\chi}_{1}^{0} \ell^{+} \ell^{-}$has an endpoint that measures $M_{\tilde{\chi}_{2}^{0}}-M_{\tilde{\chi}_{1}^{0}}$, while the distribution from the two-body decay $\tilde{\chi}_{2}^{0} \rightarrow \tilde{\ell}^{ \pm} \ell^{\mp} \rightarrow \tilde{\chi}_{1}^{0} \ell^{+} \ell^{-}$has a different shape with a sharp edge at the endpoint

$$
\sqrt{\frac{\left(M_{\tilde{\chi}_{2}^{0}}^{2}-M_{\tilde{\ell}}^{2}\right)\left(M_{\tilde{\ell}}^{2}-M_{\tilde{\chi}_{1}^{0}}^{2}\right)}{M_{\tilde{\ell}}^{2}}}
$$

Dilepton mass distributions [35] after cuts for an example of each decay are shown for ATLAS in Figs. 23, 24 and for CMS in Fig. 25. The position of the end point is $108.6 \mathrm{GeV}$ in Fig 24. The flavor-subtraction combination $e^{+} e^{-}+\mu^{+} \mu^{-}-e^{ \pm} \mu^{\mp}$ removes backgrounds from two independent decays. The last plot shows that the signal structure depends strongly on the choice of parameters. Note that at the small values of $m_{0}$ and $m_{1 / 2}$ shown, the event rates are very large. Such endpoints can be observed over a wide range of parameters as indicated in Fig. 26 63.

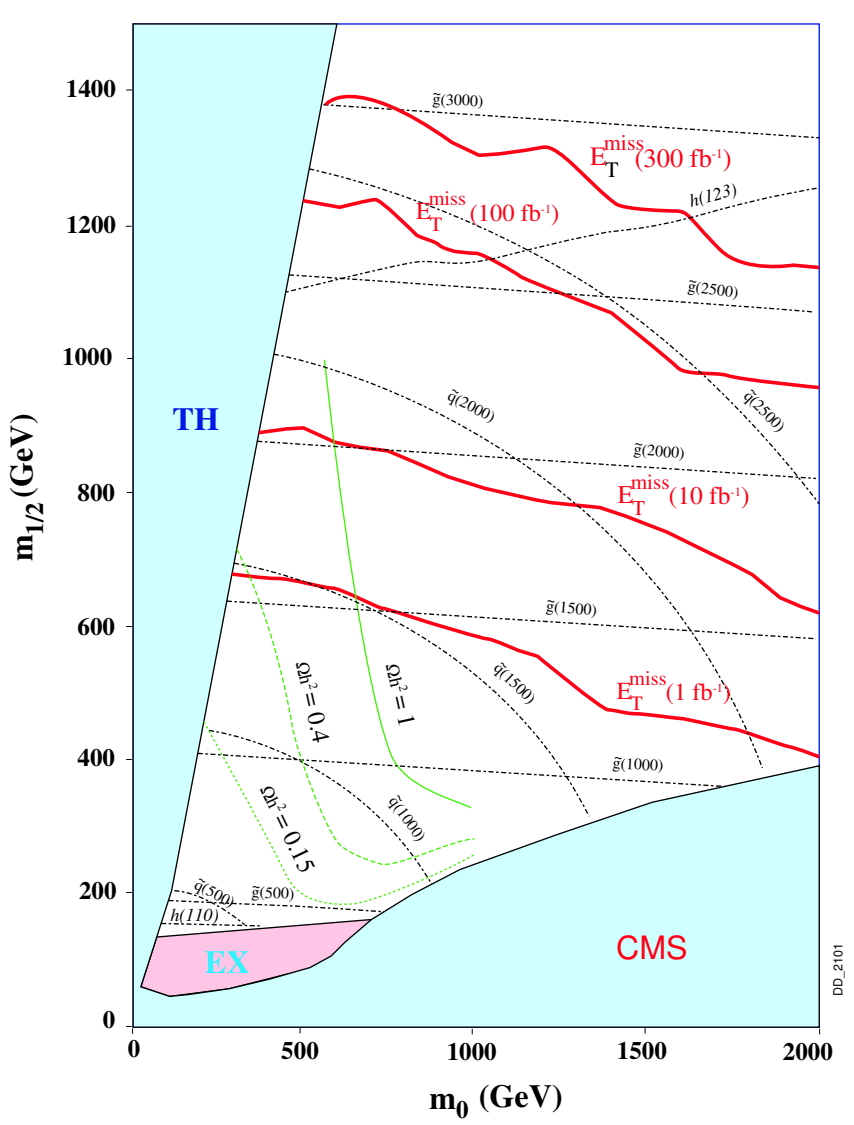

Figure 21: Plot of $5 \sigma$ reach in minimal SUGRA model for $\tan \beta=35$ and $\mu=+$ at LHC for the $\mathbb{E}_{T}$ signal for various integrated luminosities.

When a longer decay chain can be identified, more combinations of masses can be measured. Consider, for example, the decay chain

$$
\tilde{q}_{L} \rightarrow \tilde{\chi}_{2}^{0} q \rightarrow \tilde{\ell}_{R}^{ \pm} \ell^{\mp} q \rightarrow \tilde{\chi}_{1}^{0} \ell^{+} \ell^{-} q .
$$

For this decay chain, kinematics gives $\ell^{+} \ell^{-}, \ell^{+} \ell^{-} q$, and two $\ell q$ endpoints as functions of the masses. If a lower limit is imposed on the $\ell^{+} \ell^{-}$mass, there is also a minimum $\ell^{+} \ell^{-} q$ mass. With suitable cuts all of these can be measured [35, 67] for the cases considered. An example is the minimum $\ell \ell q$ mass formed from the dilepton pair shown in Fig. 27 and one of the two hardest jets. Since the hardest jets are mainly from squark decays, this smaller mass should have an endpoint given by the above decay chain at

$$
\sqrt{\frac{\left(M_{\tilde{q}_{L}}^{2}-M_{\tilde{\chi}_{2}^{0}}^{2}\right)\left(M_{\tilde{\chi}_{2}^{0}}^{2}-M_{\tilde{\chi}_{1}^{0}}^{2}\right)}{M_{\tilde{\chi}_{2}^{0}}^{2}}}
$$

In the case shown this endpoint is at $552.4 \mathrm{GeV}$. The statistical errors on the measured endpoints are typically comparable to the systematic limits, $\mathcal{O}(0.1 \%)$ for leptons and $\mathcal{O}(1 \%)$ for jets.

The set of measurements just described can be used to determine all the masses in the relevant decay chain. This is most easily done by generating the four masses at random and comparing the predicted results with the measurements. Fig. 28 


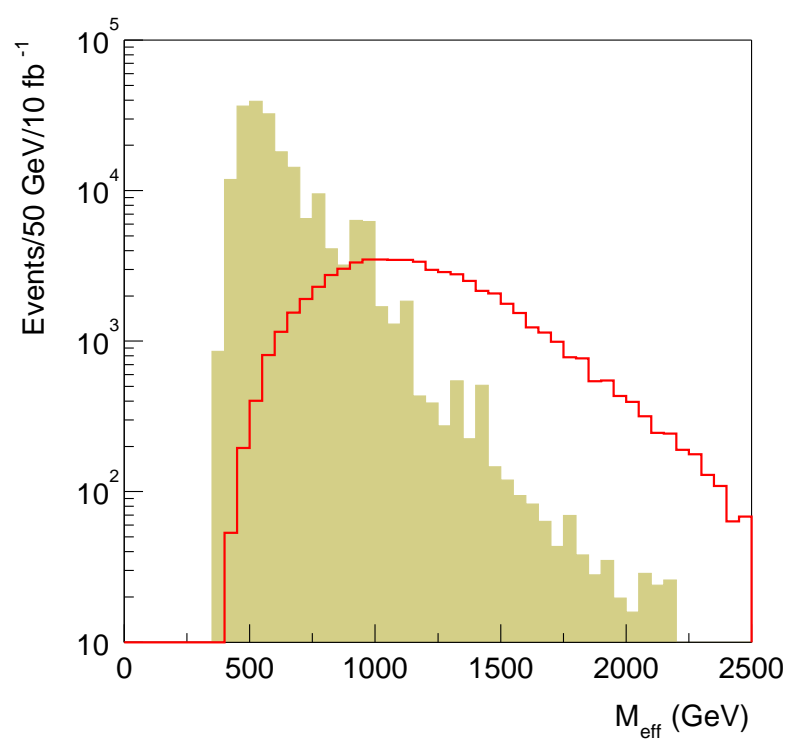

Figure 22: $M_{\text {eff }}$ distribution for a SUGRA point with gluino and squark masses of about $700 \mathrm{GeV}$ (histogram) and Standard Model background (shaded) after cuts. Based on Ref. [64].

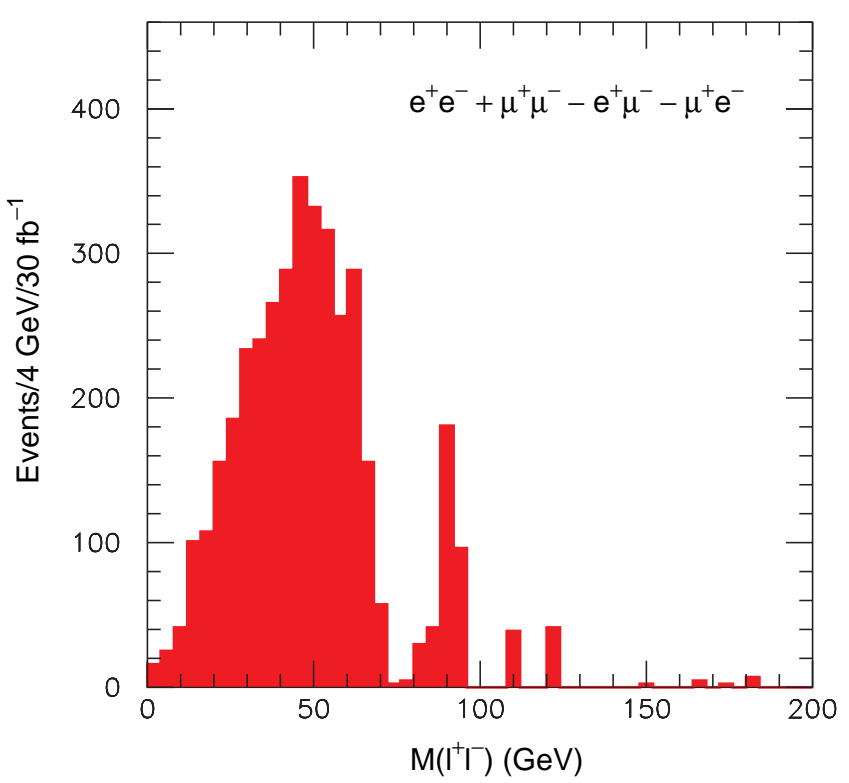

Figure 23: Plot of $e^{+} e^{-}+\mu^{+} \mu^{-}-e^{ \pm} \mu^{\mp}$ mass distribution for LHC SUGRA Point 4 with direct $\tilde{\chi}_{2}^{0} \rightarrow \tilde{\chi}_{1}^{0} \ell \ell$ decay in ATLAS. The $Z \rightarrow \ell^{+} \ell^{-}$signal comes from decays of the heavier gauginos. From Ref. [35].

shows a scatter plot of the resulting $\tilde{\ell}_{R}$ and $\tilde{\chi}_{1}^{0}$ masses for LHC SUGRA Point 5 and for a similar point in another SUSY model with this decay chain [69]. The relations between masses are determined with good precision, so these two models are easily distinguished, as can be seen in Fig. 28. Although the LSP is invisible, its mass, Fig. 29, can be measured to $\mathcal{O}(10 \%)$ through its effects on the decay kinematics.

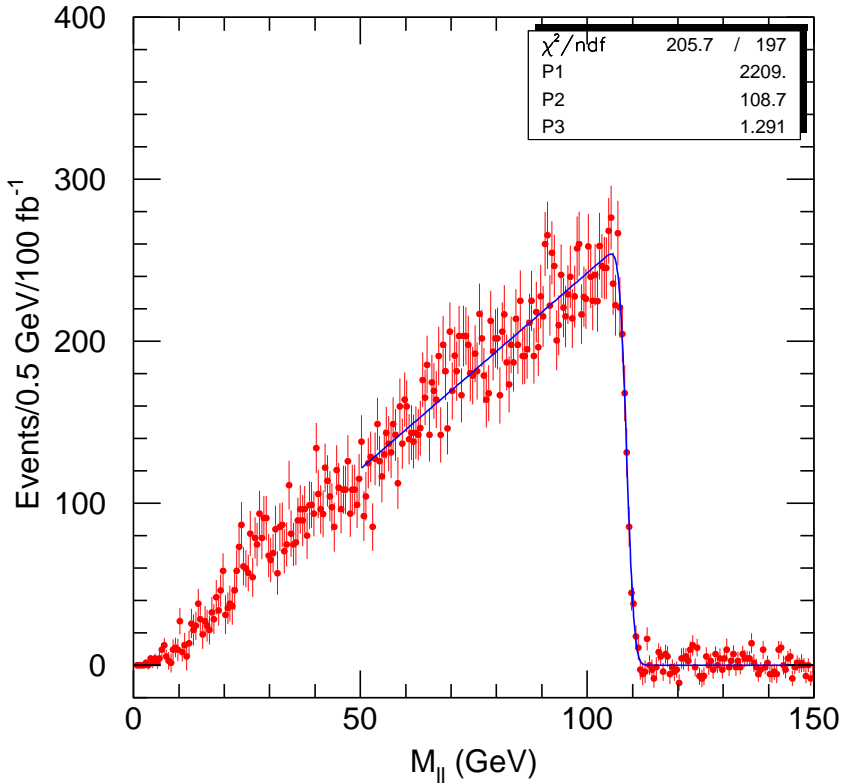

Figure 24: Plot of $e^{+} e^{-}+\mu^{+} \mu^{-}-e^{ \pm} \mu^{\mp}$ mass distribution for for LHC SUGRA Point 5 with $\tilde{\chi}_{2}^{0} \rightarrow \tilde{\ell}^{ \pm} \ell^{\mp} \rightarrow \tilde{\chi}_{1}^{0} \ell^{+} \ell^{-}$in ATLAS. From Ref. [35].
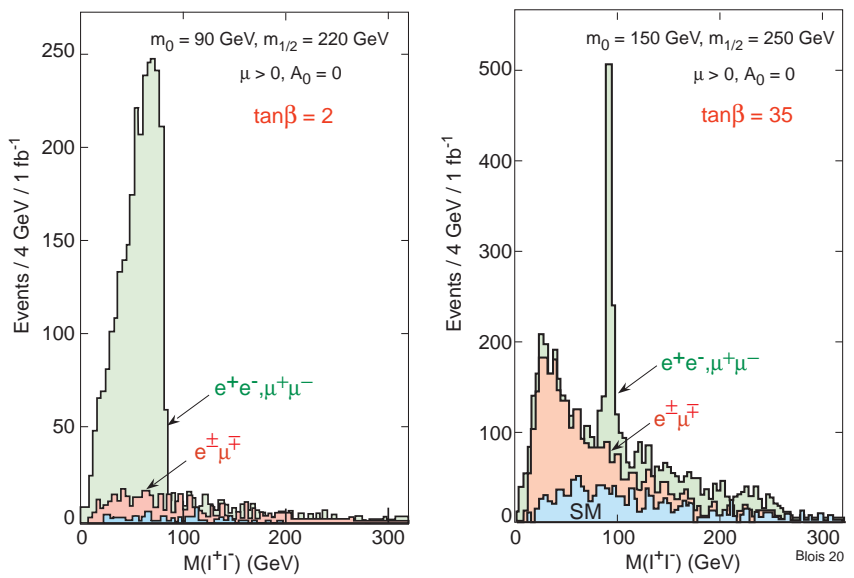

Figure 25: Plot of $e^{+} e^{-}, \mu^{+} \mu^{-}$and $e^{ \pm} \mu^{\mp}$ mass distribution for SUGRA showing the signal at two points with CMS. From Ref. [65].

If the two-body decay $\tilde{\chi}_{2}^{0} \rightarrow \tilde{\chi}_{1}^{0} h$ is open, it will typically have a substantial branching ratio; it can be dominant if the $\tilde{\chi}_{2}^{0}$ and $\tilde{\chi}_{1}^{0}$ are mainly gaugino and the slepton channel is closed. If events are selected with multiple jets, large $\mathbb{E}_{T}$, and two tagged $b$ jets, then the decay $h \rightarrow b \bar{b}$ can be reconstructed. Examples for several points with different values of $\tan \beta$ are shown in Fig. 30 [70][63]. Like the dilepton signal, this one can also be combined with additional jets to provide further information.

It is also possible that the only two-body decays are $\tilde{\chi}_{2}^{0} \rightarrow$ $\tilde{\tau}_{1} \tau \rightarrow \tilde{\chi}_{1}^{0} \tau \tau$. This can occur naturally in SUGRA if $\tilde{\chi}_{2}^{0} \rightarrow \tilde{\chi}_{1}^{0} Z$ $\tilde{\chi}_{1}^{0} h$, and $\tilde{\ell} \ell$ are all closed but $\tan \beta$ is large enough that $\tilde{\chi}_{2}^{0} \rightarrow$ $\tilde{\tau}_{1} \tau$ is open. One analysis of a sample point, LHC SUGRA Point 6, has been done [35] using hadronic $\tau$ decays to deter- 


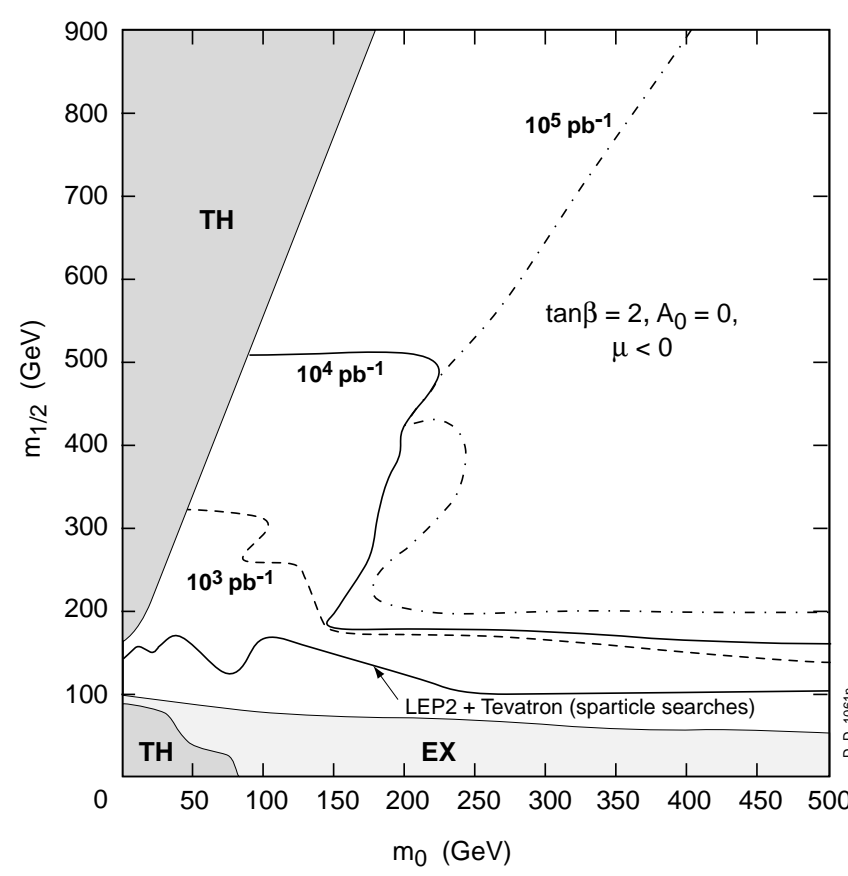

Figure 26: Reach for observing dilepton endpoints in SUGRA models with $1 \mathrm{fb}^{-1}, 10 \mathrm{fb}^{-1}$ and $100 \mathrm{fb}^{-1}$. Theory (TH) and experimental constraints are also indicated. From Ref. [63.

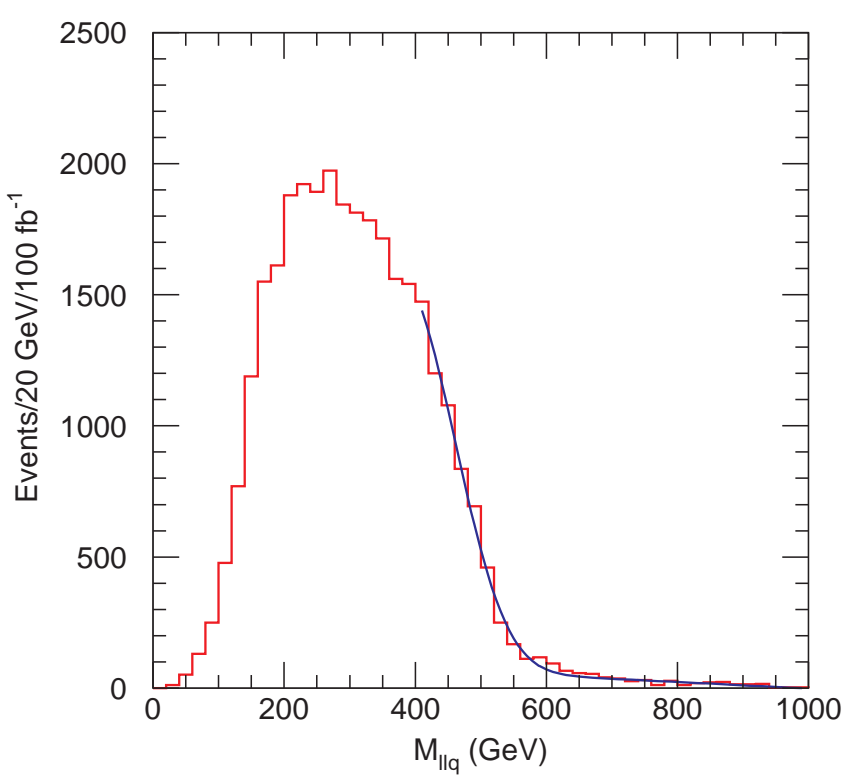

Figure 27: Plot of minimum $M(\ell \ell q)$ mass formed from $e^{+} e^{-}+$ $\mu^{+} \mu^{-}-e^{ \pm} \mu^{\mp}$ plus one of two hardest jets at LHC SUGRA Point 5. The smooth curve shows a fit used to estimate the error on the endpoint. From Ref. [35].

mine the $\tau \tau$ mass distribution. Since simple kinematic cuts select a rather pure SUSY sample with $\mathcal{O}(1)$ hadronic $\tau$ per event, the $\tau$ selection criteria were chosen not to optimize the QCD jet rejection but rather to select multi-pion decays and so to improve the $\tau \tau$ mass resolution. The combination $\tau^{+} \tau^{-}-\tau^{ \pm} \tau^{ \pm}$

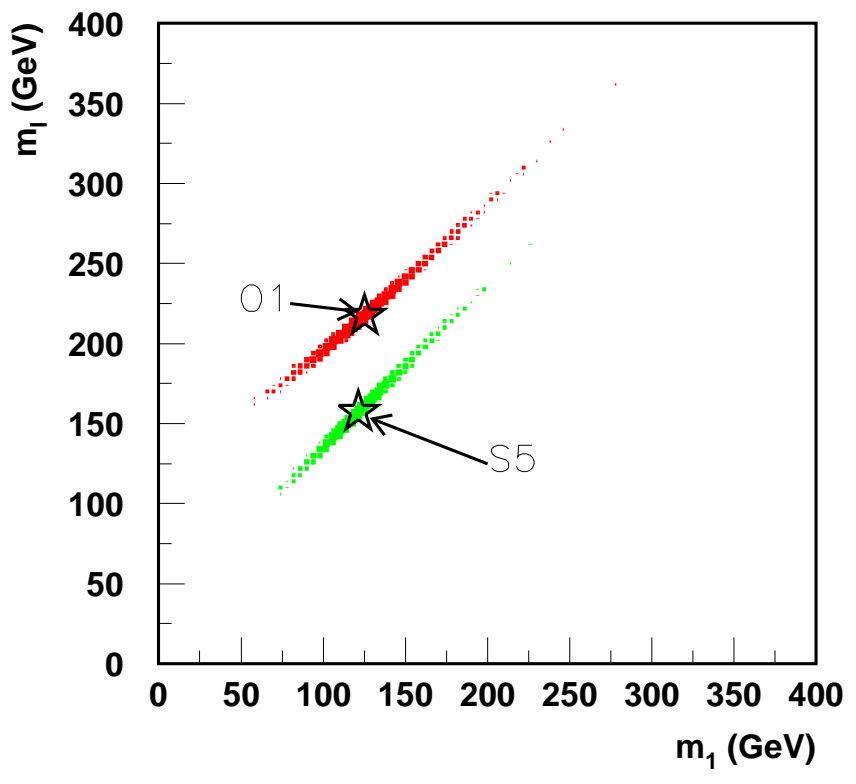

Figure 28: Scatter plot of reconstructed values of $m_{\ell} \equiv M_{\tilde{\ell}_{R}}$ vs. $m_{1} \equiv M_{\tilde{\chi}_{1}^{0}}$ for LHC Point 5 (S5) and for an "optimized string model" (O1) using multiple measurements from the decay chain $\tilde{q}_{L} \rightarrow \tilde{\chi}_{2}^{0} q \rightarrow \tilde{\ell}_{R}^{ \pm} \ell^{\mp} q \rightarrow \tilde{\chi}_{1}^{0} \ell^{+} \ell^{-} q$. The stars mark the input values. From Ref. [69].

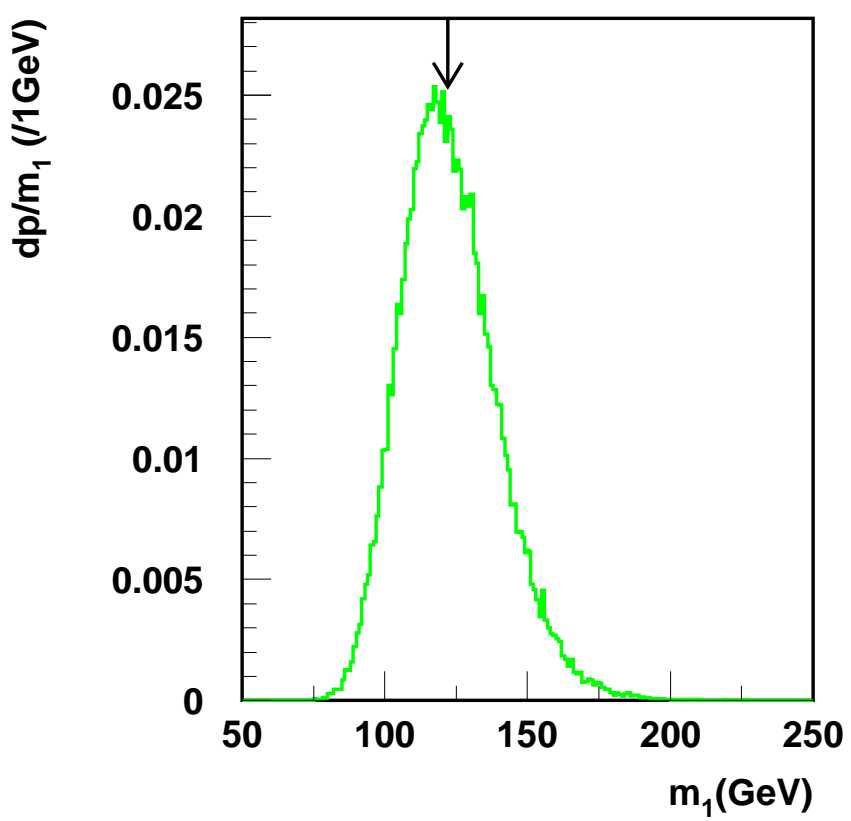

Figure 29: Projection of $M_{\tilde{\chi}_{1}^{0}}$ in Fig. 28 for LHC Point 5. From Ref. [69].

removes most of the background from misidentified jets. The resulting visible mass distribution is shown in Fig. 31. If $\tau$ 's could be measured perfectly, this distribution would have a shape like Fig. 24 with a sharp endpoint at $59.6 \mathrm{GeV}$. Although the endpoint is shifted and broadened by the missing neutrinos, measurements at the $\sim 5 \%$ level seem possible even in this difficult case. (This point and similar ones would give a very large 

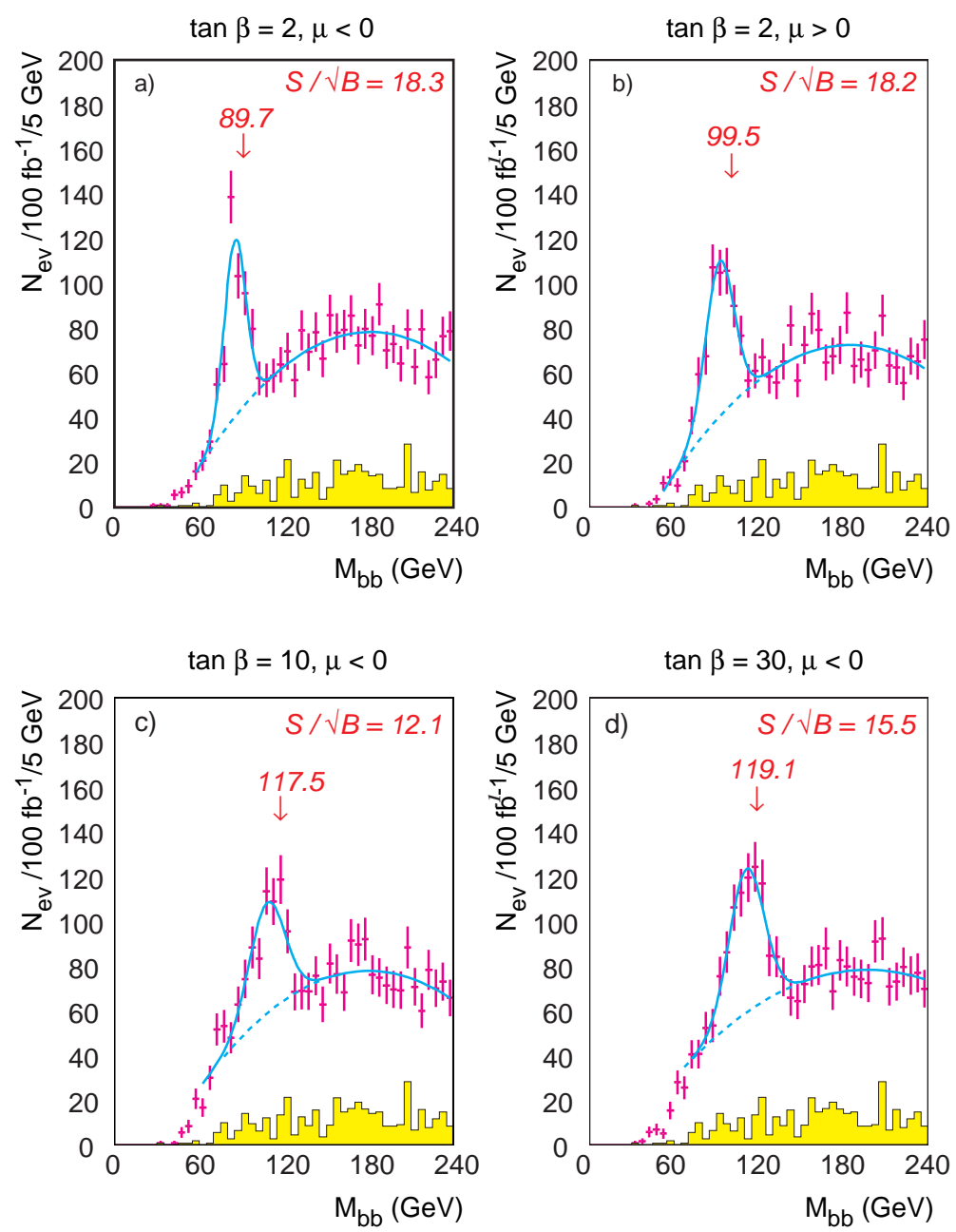

Figure 30: Plot of $b \bar{b}$ dijet mass distribution (points) with $h \rightarrow b \bar{b}$ signal (solid), SUSY background (dashed), and Standard Model background (shaded) for various $\tan \beta$. From Ref. [70] [63] .

contribution to $g_{\mu}-2$ in contradiction to Ref. [68].)

Kinematic endpoints are of course only a small part of the data that will be available from the LHC if SUSY is discovered. One will be able to measure cross sections, relative branching ratios, and many other kinematic distributions. For example, in the decay chain $\tilde{\chi}_{2}^{0} \rightarrow \tilde{\ell}_{R}^{ \pm} \ell^{\mp} \rightarrow \tilde{\chi}_{1}^{0} \ell^{+} \ell^{-}$, the ratio $p_{T, 2} / p_{T, 1}$ of the two leptons contains information that is independent of the endpoint: one lepton will be soft if the slepton is nearly degenerate with either the $\tilde{\chi}_{2}^{0}$ or the $\tilde{\chi}_{1}^{0}$.

\section{B. GMSB Measurements}

In GMSB models the gravitino $\tilde{G}$ is very light; the phenomenology is determined by the nature of the next lightest SUSY particle (NLSP), either the $\tilde{\chi}_{1}^{0}$ or a slepton, and by its lifetime to decay into a $\tilde{G}$. GMSB models generally provide additional experimental handles and so are easier to analyze than SUGRA models.

If the NLSP is the $\tilde{\chi}_{1}^{0}$ and it decays promptly, $\tilde{\chi}_{1}^{0} \rightarrow \tilde{G} \gamma$, then SUSY events contain two hard, isolated photons in addition to $\mathbb{E}_{T}$, jets, and perhaps leptons. The decay chain $\tilde{\chi}_{2}^{0} \rightarrow \tilde{\ell}^{ \pm} \ell^{\mp} \rightarrow$ $\tilde{\chi}_{1}^{0} \ell^{+} \ell^{-} \rightarrow \tilde{G} \ell^{+} \ell^{-} \gamma$ provides, in addition to an $\ell^{+} \ell^{-}$endpoint like Fig. 24, precisely measurable $\ell \ell \gamma$ and $\ell \gamma$ endpoints. An example is shown in Fig. 32. These measurements alone allow the masses involved to be determined precisely [35].

If the NLSP is a $\tilde{\tau}$ and is long-lived, then it penetrates the calorimeter like a high momentum muon but has $\beta<1$. The $\tilde{\tau}$ mass can be measured directly using the muon chambers as a time-of-flight system [35, 71]; see Fig. 33. Once this mass is known, all the other masses can be determined directly by observing mass peaks [35].

The lifetime of the NLSP measures the overall SUSY breaking scale and so is a crucial parameter in GMSB models. For a $\tilde{\chi}_{1}^{0}$ NLSP with a very short lifetime, the Dalitz decay $\tilde{\chi}_{1}^{0} \rightarrow$ $\tilde{G} e^{+} e^{-}$can be used; the reach is limited only by the resolution of the vertex detector. A long-lived $\tilde{\chi}_{1}^{0}$ that decays inside the tracker will produce a photon that does not point to the primary vertex. The ATLAS electromagnetic calorimeter provides pointing and can detect such decays for $c \tau \lesssim 100 \mathrm{~km}[35]$. The lifetime of a $\tilde{\tau}$ can be measured for $1 \lesssim c \tau \lesssim 100 \mathrm{~m}$ by counting the numbers of events with one and two reconstructed sleptons 72 . It should also be possible to reconstruct $\tilde{\tau} \rightarrow \tilde{G} \tau$ 


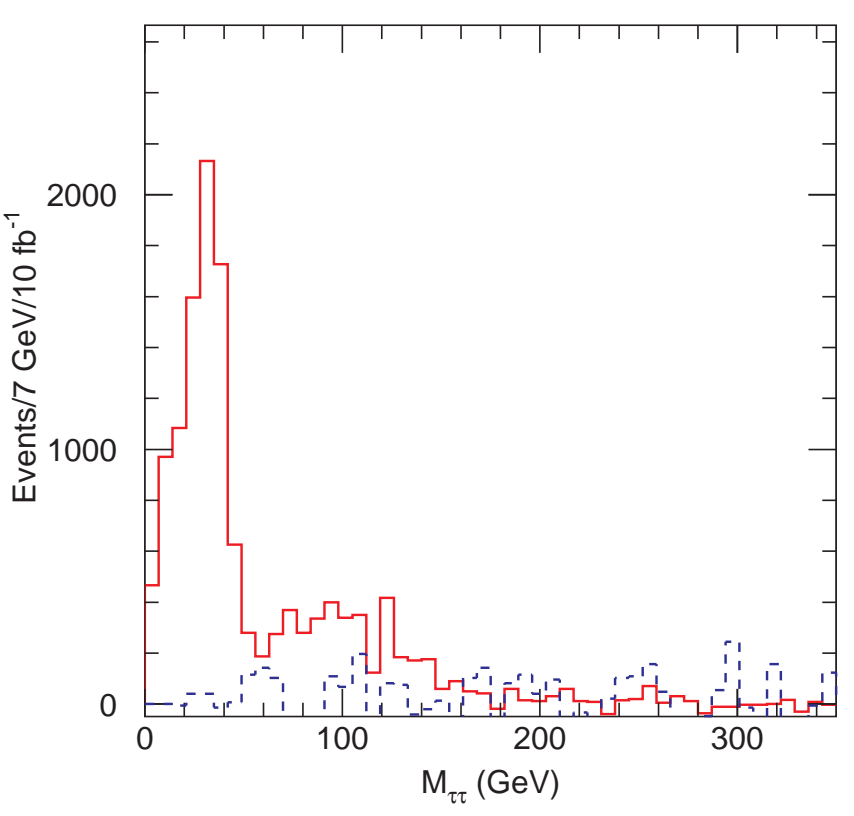

Figure 31: Plot of $\tau^{+} \tau^{-}-\tau^{ \pm} \tau^{ \pm}$visible mass distribution with hadronic $\tau$ decays at LHC SUGRA Point 6. From Ref. [35].

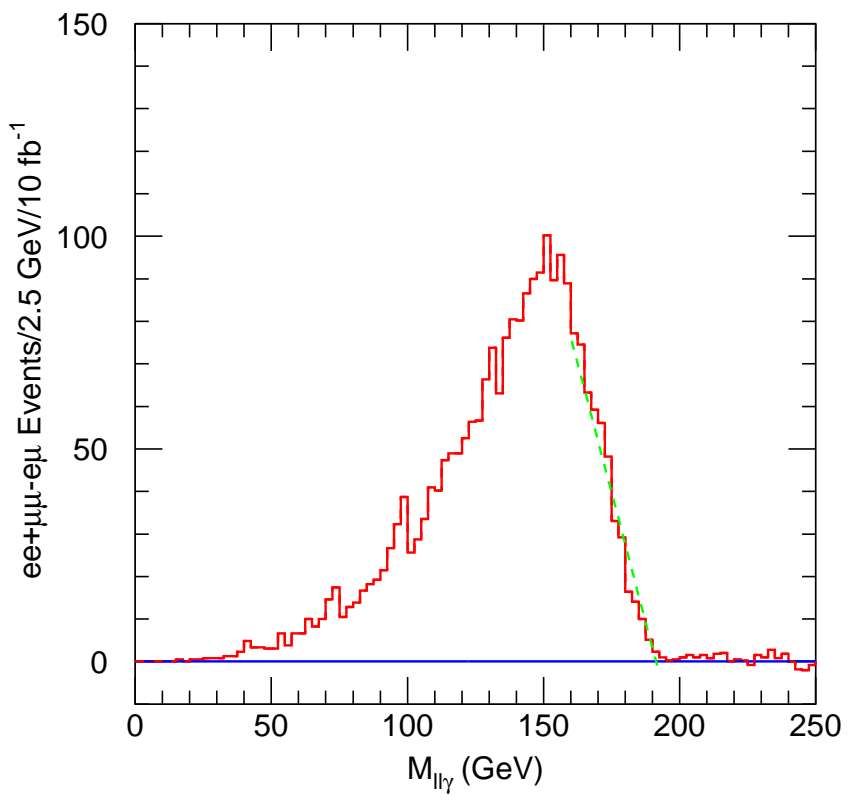

Figure 32: $\ell^{+} \ell^{-} \gamma$ mass distribution for a GMSB point with a prompt $\tilde{\chi}_{2}^{0} \rightarrow \tilde{\ell}_{R}^{ \pm} \ell^{\mp} \rightarrow \tilde{\chi}_{1}^{0} \ell^{+} \ell^{-} \rightarrow \tilde{G} \gamma \ell^{+} \ell^{-}$decay. From Ref. [35].

decays in the central tracker.

\section{AMSB Measurements}

In the AMSB model the $\tilde{\chi}_{1}^{ \pm}$and (mainly wino) $\tilde{\chi}_{1}^{0}$ are almost degenerate, while the (mainly bino) $\tilde{\chi}_{2}^{0}$ is heavier. Hence, signatures involving $\tilde{\chi}_{2}^{0}$ decays are largely unchanged from similar SUGRA ones [73]. Typically, the splitting between the $\tilde{\chi}_{1}^{ \pm}$and $\tilde{\chi}_{1}^{0}$ is a few hundred $\mathrm{MeV}$, so the chargino decays via

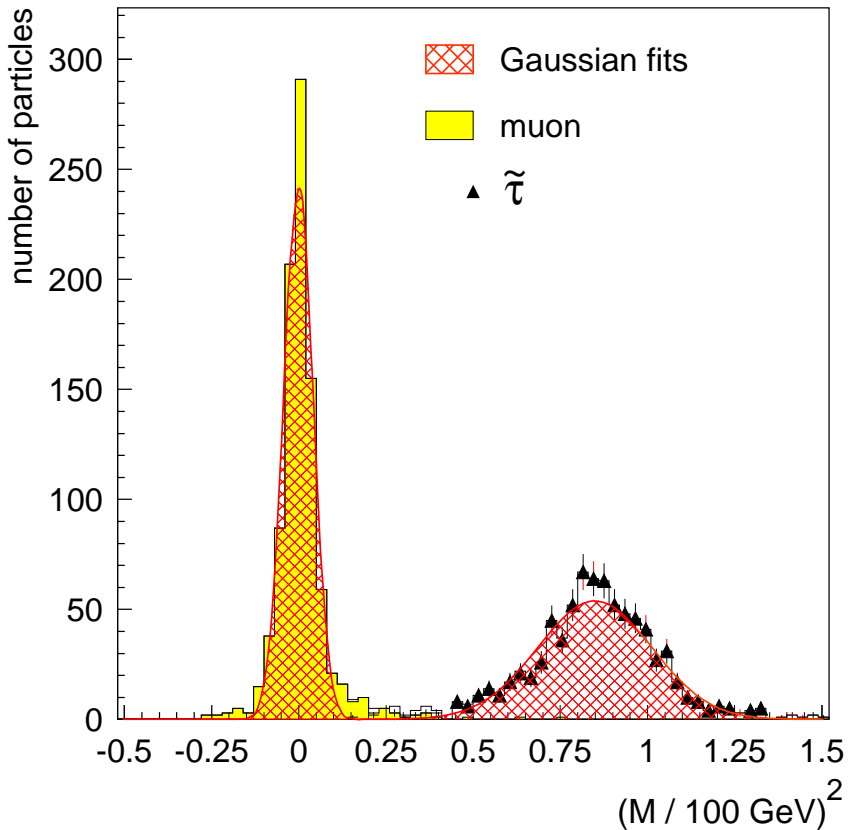

Figure 33: Muon and $\tilde{\tau}_{1}$ masses reconstructed by time of flight. From Ref. [71].

$\tilde{\chi}_{1}^{ \pm} \rightarrow \tilde{\chi}_{1}^{0} \pi^{ \pm}$with $c \tau \sim 1 \mathrm{~cm}$ and is mostly invisible. The fraction of single lepton events is consequently reduced. A small fraction of the $\tilde{\chi}_{1}^{ \pm}$will travel far enough to be seen in the vertex detectors.

\section{D. $R$-Parity Violation}

The SUGRA, GMSB, and AMSB models assume that $R$ parity is conserved so that the LSP is stable. It is possible that either baryon number or lepton number is violated, allowing the LSP to decay; violation of both would allow rapid proton decay. If lepton number is violated, then SUSY events will contain multiple leptons, e.g., from $\tilde{\chi}_{1}^{0} \rightarrow \ell^{+} \ell^{-} \nu$ or $\tilde{\chi}_{1}^{0} \rightarrow \ell q \bar{q}$. These cases are easy to detect, and similar partial reconstruction techniques can be used [35].

If baryon number is violated, the LSP will decay into jets, $\tilde{\chi}_{1}^{0} \rightarrow q q q$, giving events with very high jet multiplicity and no (large) $\mathbb{E}_{T}$. The QCD background for this is not well known, but it appears difficult to extract the signal using only jets. It is possible, however, to reconstruct SUSY events using cascade decays involving leptons. The results for an analysis at a point with the decay chain $\tilde{\chi}_{2}^{0} \rightarrow \tilde{\ell}^{ \pm} \ell^{\mp} \rightarrow \tilde{\chi}_{1}^{0} \ell^{+} \ell^{-} \rightarrow$ $q q q \ell^{+} \ell^{-}$is shown in Fig. 34. The mass combinations $m^{ \pm}=$ $M\left(q q q \ell^{+} \ell^{-}\right) \pm M(q q q)$ show clear peaks corresponding to the $\tilde{\chi}_{1}^{0}$ and $\tilde{\chi}_{2}^{0}$ masses. The $\tilde{\chi}_{2}^{0}, \tilde{\ell}_{R}$, and $\tilde{\chi}_{1}^{0}$ masses can be determined from these plus a dilepton edge similar to Fig. 24 .

\section{E. Decay of Higgs to Sparticles}

For certain choices of the MSSM parameters, it is possible for the heavy Higgs bosons $A$ and $H$ to decay to sparticles. As an example, The decay $A, H \rightarrow \tilde{\chi}_{2}^{0} \tilde{\chi}_{2}^{0}$ has been investigated by both collaborations. The subsequent decay $\tilde{\chi}_{2}^{0} \rightarrow \ell^{+} \ell^{-} \tilde{\chi}_{1}^{0}$ gives 

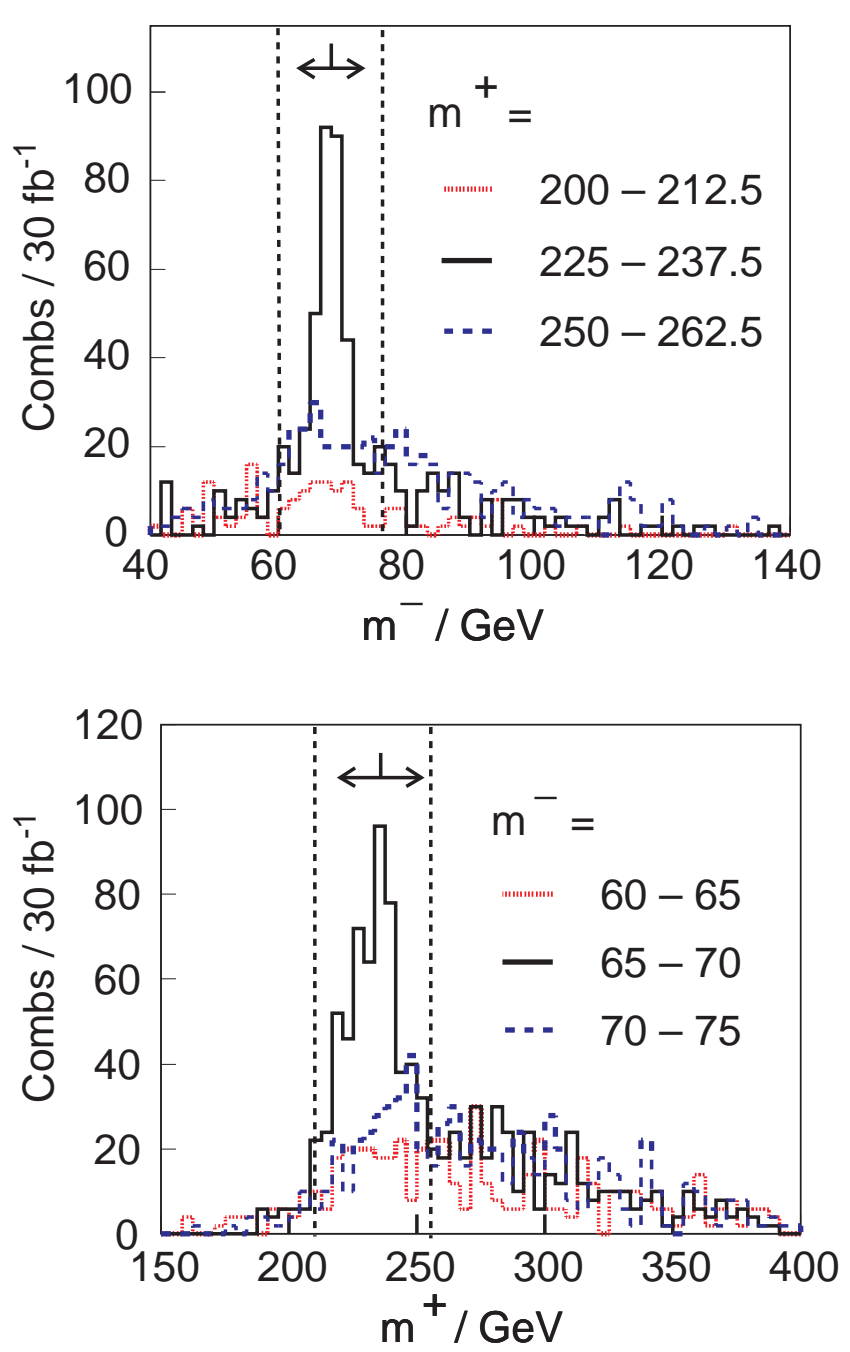

Figure 34: Distributions of $m^{ \pm}=M\left(\ell^{+} \ell^{-} q q q\right) \pm M(q q q)$ in $R$-parity violating SUSY events with $\tilde{\chi}_{1}^{0} \rightarrow q q q$ after selecting either the peak or sidebands in $m^{\mp}$. From Ref. [74].

rise to events with four isolated leptons. The invariant mass of the 4-lepton system for one such case is shown in Fig. 35. Here the study [75] is done in the context of the MSSM.

This signal is visible over a large fraction of parameter space as can be seen from Fig. 36 which shows the accessible region in the SUGRA model for various values of $m_{0}$. Note that the value of $m_{1 / 2}$ is determined once $M_{A}$ and $m_{0}$ are given. For large values of $M_{A}$, the decay $A, H \rightarrow t \bar{t}$ dominates and the signal is unobservable.

\section{F. SUSY Summary}

If SUSY with $R$ parity conservation exists at the TeV scale, then observation of $\mathbb{E}_{T}$ plus multijet signatures with the ATLAS and CMS detectors at the LHC should be straightforward. Many GMSB models provide additional handles. If lepton number is violated, the signatures are easier. If baryon number is violated, discovery probably must rely on selecting particular cascade decays, although measurements are then easier. The kinemat-

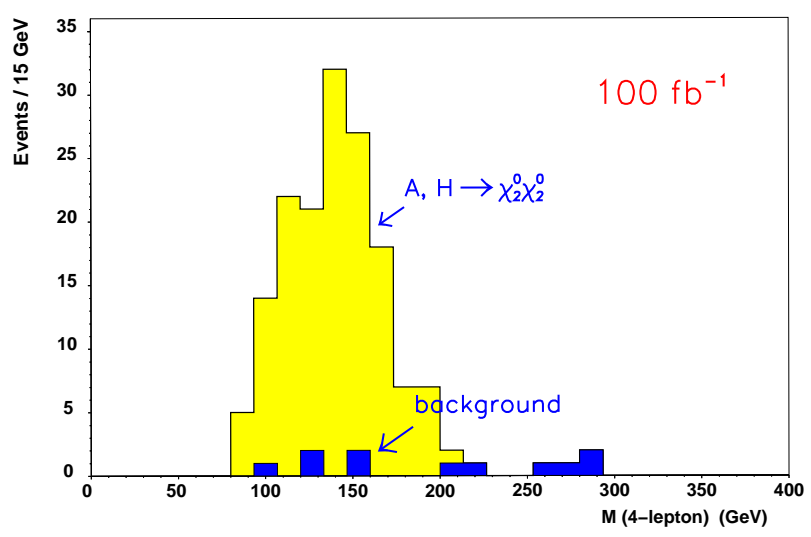

Figure 35: The invariant mass distribution of $\ell^{+} \ell^{-} \ell^{+} \ell^{-}$for leptons arising from the decay $A \rightarrow \tilde{\chi}_{2}^{0} \tilde{\chi}_{2}^{0}$ for the MSSM with $M_{2}=120 \mathrm{GeV}, M_{1}=60 \mathrm{GeV}, \mu=500 \mathrm{GeV}$ and $m_{\tilde{l}}=250$ GeV. From Ref. [75].

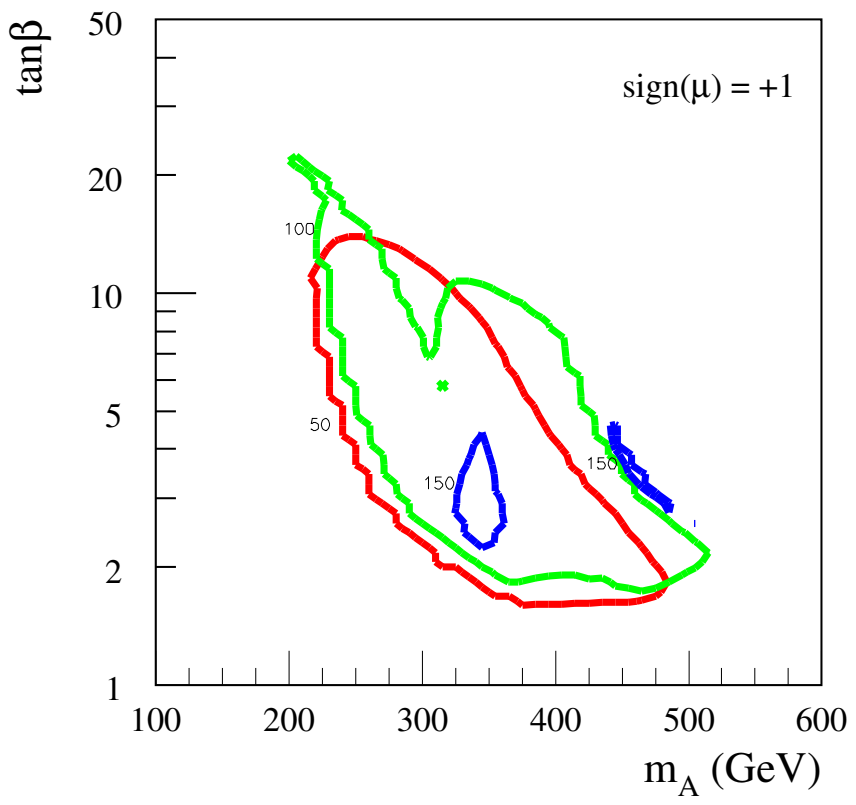

Figure 36: The region in $M_{A}-\tan \beta$ where the decay $A \rightarrow$ $\tilde{\chi}_{2}^{0} \tilde{\chi}_{2}^{0} \rightarrow 4 \ell+X$ is observable in the SUGRA model. The contours are labeled by $m_{0}$. An integrated luminosity of $300 \mathrm{fb}^{-1}$ is assumed. From Ref. [35].

ics and qualitative features of the discovery signatures can be used to establish the approximate mass scale and to distinguish classes of models.

If $R$ parity is conserved, then all SUSY decays contain a missing LSP, so no mass peaks can be reconstructed. Kinematic endpoints of mass distributions have proved useful for a number of SUSY points in a variety of SUSY models [35]. The method seems fairly general: there is usually at least one distinctive mode - typically $\tilde{\chi}_{2}^{0} \rightarrow \tilde{\chi}_{1}^{0} \ell^{+} \ell^{-}, \tilde{\chi}_{2}^{0} \rightarrow \tilde{\ell}_{R}^{ \pm} \ell^{\mp}$, or $\tilde{\chi}_{2}^{0} \rightarrow \tilde{\chi}_{1}^{0} h \rightarrow \tilde{\chi}_{1}^{0} b \bar{b}-$ from which to start. These can be com- 
bined with jets to determine other combinations of masses.

The SUSY events will contain much more information than just endpoints like those described above. For example, while it is not possible to reconstruct $\tilde{\chi}_{1}^{ \pm}$decays in the same way because of the missing neutrino, one can get information about the chargino mass by studying $M_{\ell q}$ and other distributions for 1-lepton events. Cross sections and branching ratios can also be measured; interpretation of these will be limited by the theoretical errors on the calculation of cross sections and acceptances. Without real experimental data, it is difficult to assess such theoretical systematic errors.

This program will provide a large amount of information about gluinos, squarks, and their main decay products, including $\tilde{\chi}_{1}^{0}, \tilde{\chi}_{2}^{0}, \tilde{\chi}^{ \pm}$, and any sleptons that occur in their decays. The heavy gauginos typically have small cross sections, as do sleptons produced only by the Drell-Yan process. High precision measurements of the LSP mass and of couplings and branching ratios also appear more difficult.

\section{STRONG EWSB DYNAMICS}

While the existing precision electroweak measurements are consistent with a light Higgs boson, the possibility of electroweak symmetry breaking by new strong dynamics at the TeV scale cannot be excluded.

\section{A. Strongly interacting $W^{\prime}$ s}

The couplings of longitudinally polarized gauge bosons to each other are fixed at low energy by the nature of the spontaneously broken electroweak symmetry and are independent of the details of the breaking mechanism. Scattering amplitudes calculated from these couplings will violate unitarity at center of mass energies of the $W W$ system around $1.5 \mathrm{TeV}$. New physics must enter to cure this problem. In the minimal Standard Model and its supersymmetric version, the cure arises from the weakly coupled Higgs bosons. If no Higgs-like particle exists, then new non-perturbative dynamics must enter in the scattering amplitudes for $W W, W Z$ and $Z Z$ scattering at high energy. Therefore, if no new physics shows up at lower mass scales, one must be able to probe $W_{L} W_{L}$ scattering at $\sqrt{\hat{s}} \sim 1 \mathrm{TeV}$.

Various models exist that can be used as benchmarks for this physics [76]. The basic signal for all of these models is an excess of events over that predicted by the Standard Model for gauge boson pairs of large invariant mass. In certain models resonant structure can be seen; an example of this is given in the next subsection. Since in the Standard Model there is no process $q \bar{q} \rightarrow W^{ \pm} W^{ \pm}$, the $W^{ \pm} W^{ \pm}$final state expected to have a much smaller background than the $Z Z$ or $W^{+} W^{-}$ones. There are small $W^{ \pm} W^{ \pm}$backgrounds from higher order processes and from $W Z$ if one lepton is lost. The background from charge misidentification is negligible in either ATLAS or CMS.

ATLAS [35] conducted a study of the signal and background in this channel. Events were selected that have two leptons of the same sign with $p_{T}>40 \mathrm{GeV}$ and $|\eta|<1.75$. If a third lepton was present that, in combination with one of the other two, was consistent with the decay of a $Z$ (mass within $15 \mathrm{GeV}$ of the $Z$ mass), the event was rejected. This cut is needed to

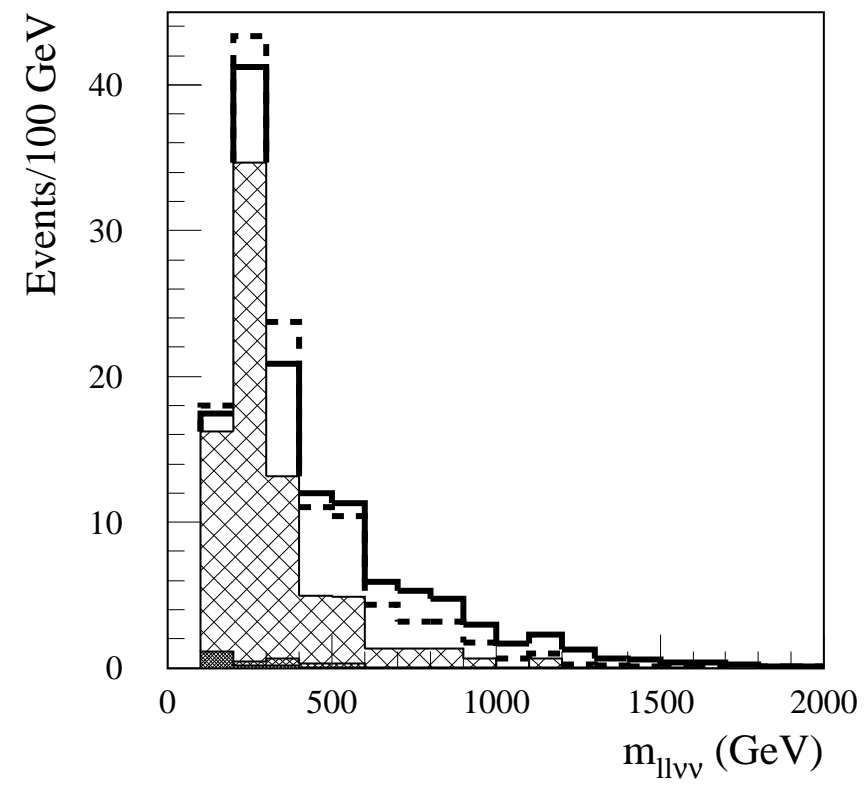

Figure 37: The invariant mass spectrum for same sign dileptons in the search for a strongly coupled $W W$ sector as simulated by ATLAS. The signal corresponds to a $1 \mathrm{TeV}$ Higgs boson. The backgrounds are from $W Z$ and $W_{T} W_{T}$ production via electroweak bremsstrahlung. From Ref. [35].

eliminate the background from $W Z$ and $Z Z$ final states. In addition the two leptons are required to have invariant mass above $100 \mathrm{GeV}$ and to be separated in $\phi$ so that $\cos \phi<-0.5$. At this stage, there are $\sim 1700$ Standard Model events for a luminosity of $300 \mathrm{fb}^{-1}$. Of these events roughly $90 \%$ are from $W Z$ and $Z Z$ final states and $10 \%$ from $W t \bar{t}$. There are of order 300 signal events depending upon the model used for the strongly coupled gauge boson sector. Additional cuts are needed to reduce the background. A jet veto requiring no jets with $p_{T}>50$ $\mathrm{GeV}$ and $|\eta|<2$ is effective against the $W t \bar{t}$ final state. The requirement of two forward jet tags each with $15<p_{T}<150$ $\mathrm{GeV}$ and $|\eta|>2$ reduces the $W W, Z Z$ and $W Z$ background.

The remaining background of $\sim 80$ events is dominated by the $q \bar{q} \rightarrow W W q \bar{q}$ processes. The signal rates vary between 35 and 9 events depending upon the model. The largest rate arises from a model where the $W W$ scattering amplitude, which is known at small values of $\sqrt{s}$ from low energy theorems, is extrapolated until it saturates unitarity and its growth is then cut off. The case of a $1 \mathrm{TeV}$ Standard Model Higgs boson is shown in Fig. 37 where there are approximately 20 events. It can be seen that the signal and background have the same shape; therefore the establishment of a signal requires confidence in the expected level of the background. The experiment is very difficult, but at full luminosity, a signal might be extracted by comparing the rate for $W^{+} W^{+}$with those for $W Z, W^{+} W^{-}$, and $Z Z$ final states.

A similar study in CMS of the $W^{+} W^{+}$final state leads to a similar conclusion [77]. Jet tagging (vetoing) in the forward (central) region is essential to extract a signal. 

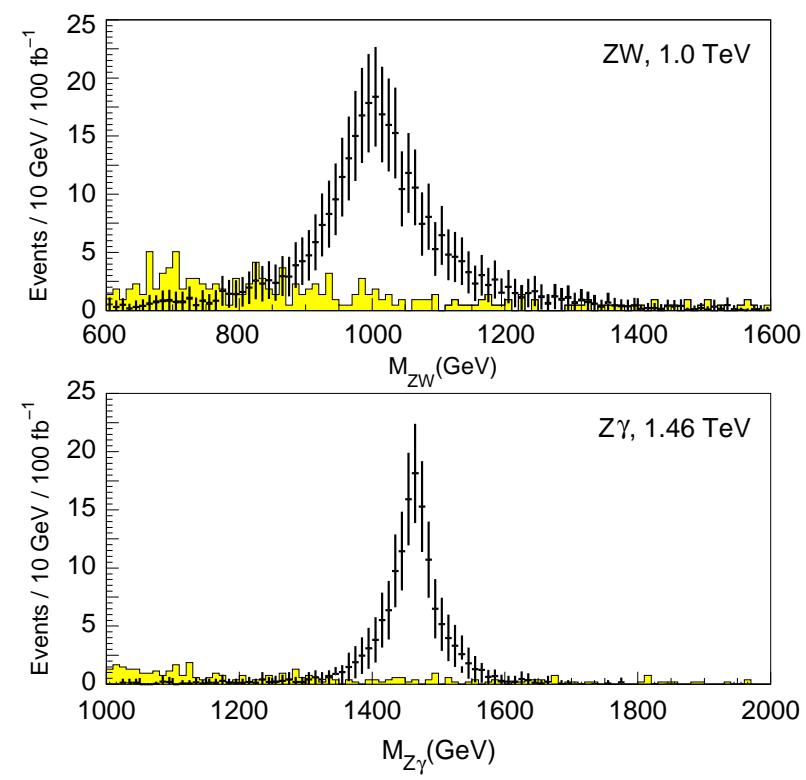

Figure 38: Reconstructed masses for high-mass resonances decaying into gauge boson pairs a simulated by ATLAS: $(a) \rho_{T}$ of mass $1.0 \mathrm{TeV}$ decaying into $W Z$ and subsequently into 3 leptons; and $(b) \omega_{T}$ of mass $1.46 \mathrm{TeV}$ decaying into $Z \gamma$ with $Z \rightarrow 2$ leptons. From Ref. [1].

\section{B. Technicolor}

Many models of strong electroweak symmetry breaking (technicolor [78] 79], topcolor-assisted technicolor [80], BESS [81]) predict resonances which decay into vector bosons (or their longitudinal components). These signals are very striking since they are produced with large cross sections and may be observed in the leptonic decay modes of the $W$ and $Z$ where the backgrounds are very small.

ATLAS has studied a techni-rho, $\rho_{T} \rightarrow W Z$, with $W \rightarrow \ell \nu$, $Z \rightarrow \ell \ell$, for $m_{\rho_{T}}=1.0 \mathrm{TeV}$ and also a techni-omega, $\omega_{T} \rightarrow$ $Z \gamma$, with $Z \rightarrow \ell \ell$, for $m_{\omega_{T}}=1.46 \mathrm{TeV}$. The backgrounds due to $t \bar{t}$ and continuum vector-boson pair production are small as can be seen in Fig. 38.

More challenging are the possible decays into non-leptonic modes such as $\rho_{T} \rightarrow W(\ell \nu) \pi_{T}(b \bar{b})$, which has a signature like associated $W H$ production with $H \rightarrow b \bar{b} ; \eta_{T} \rightarrow t \bar{t}$, for which the signature is a resonance in the $t \bar{t}$ invariant mass; and $\rho_{T 8} \rightarrow$ jet jet, for which the signature is a resonance in the dijet invariant mass distribution.

\section{Compositeness}

If quarks have substructure, it will be revealed in the deviations of the jet cross-section from that predicted by QCD. The deviation is parameterized by an interaction of the form

$$
\frac{4 \pi}{\Lambda^{2}} q \gamma^{\mu} \bar{q} q \gamma^{\mu} \bar{q}
$$

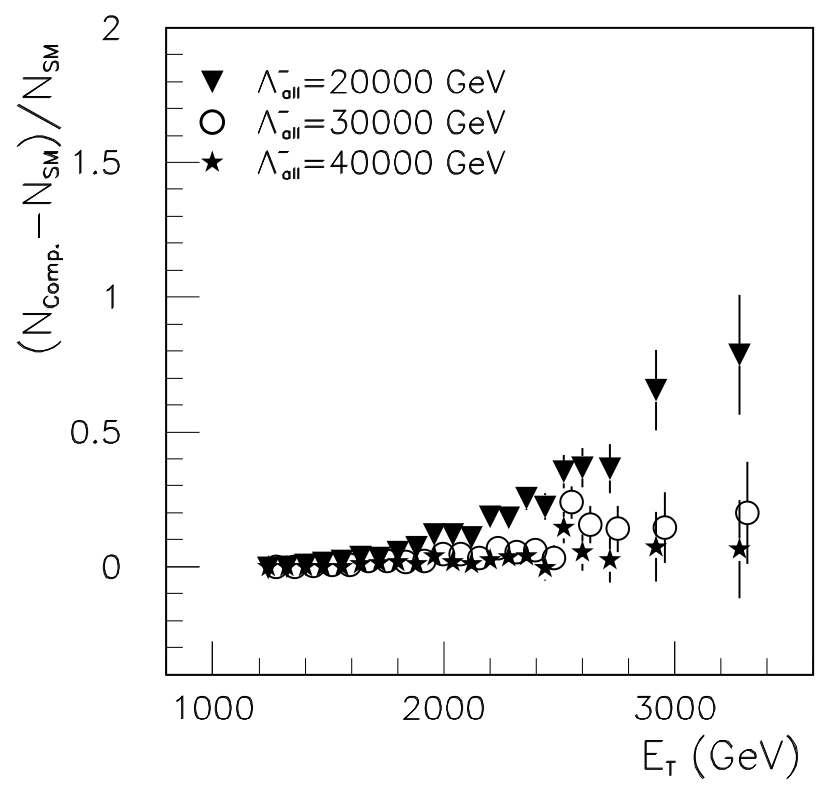

Figure 39: Difference of the Standard Model prediction and the effect of compositeness on the jet $E_{T}$ distribution, normalized to the Standard Model rate. The errors correspond to $300 \mathrm{fb}^{-1}$ for various values of the compositeness scale $\Lambda$. From Ref. [35].

which is strong at a scale $\Lambda$. This is regarded as an effective interaction which is valid only for energies less than $\Lambda$. The ATLAS collaboration has investigated the possibilities for searching for structure in the jet cross-section at high $p_{T}$. Fig. 39 shows the normalized jet cross section $d \sigma / d p_{T} d \eta$ at $\eta=0$. The rate is shown as a function of $p_{T}$ for various values of $\Lambda$ and is normalized to the value expected from QCD. The error bars at a particular value of $p_{T}$ indicate the size of the statistical error to be expected at that value for luminosities of $300 \mathrm{fb}^{-1}$. It can be seen that the LHC at full luminosity will be able to probe up to $\Lambda=20 \mathrm{TeV}$ if the systematic uncertainties are smaller than the statistical ones. Systematic effects are of two types; theoretical uncertainties in calculating the QCD rates and detector effects. The former are dependent upon an accurate knowledge of the structure functions in the $x$ range of interest and upon higher order QCD corrections to the jet cross-sections. Uncertainties from these sources can be expected to be less than $10 \%$.

Experimental uncertainties are of two types: mismeasurement due to resolution and nonlinearities in the detector response. The former are at the $20 \%$ level; the latter can be more serious and can induce changes in the apparent shape of the jet crosssection. In the case of ATLAS these non-linearities could fake a compositeness effect with a scale $\Lambda \sim 30 \mathrm{TeV}$, which is beyond the limit of sensitivity.

The angular distribution of the jets in a dijet event selected so that the dijet pair has a very large mass is less sensitive to the non-linearities. Events are selected with the invariant mass of the jet pair is above some $M_{0}$, and the variable $\chi$ defined by $\chi=(1+\cos \theta) /(1-\cos \theta)$ where $\theta$ is the angle of an outgoing jet relative to the beam direction in the center of mass frame of 


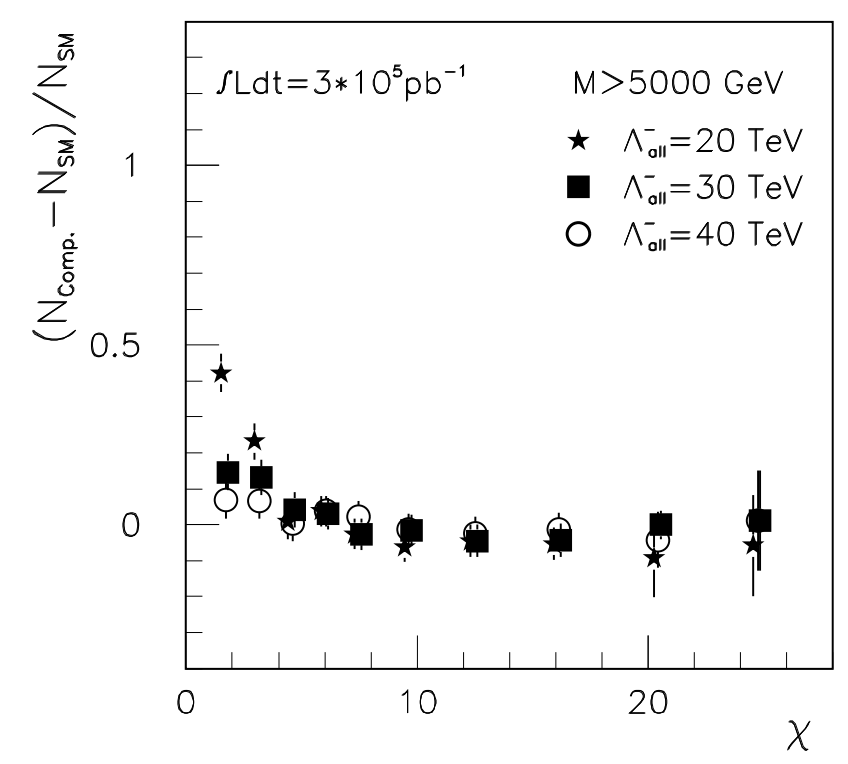

Figure 40: Difference of the Standard Model prediction and the effect of compositeness on the di-jet angular distribution for di-jet mass above $5000 \mathrm{GeV}$, normalized to the Standard Model rate. The errors correspond to $300 \mathrm{fb}^{-1}$ for various values of the compositeness scale $\Lambda$. From Ref. [35].

the jet pair. The distribution shown in Fig. 40 illustrates that $\Lambda \sim 40 \mathrm{TeV}$ is accessible via this variable.

A better constraint on the scale $\Lambda$ may be obtained from DrellYan dilepton final states, if leptons and quarks are both composite and share common constituents.

\section{NEW GAUGE BOSONS}

A generic prediction of superstring theories is the existence of additional $U(1)$ gauge groups. There is thus motivation to search for additional $W^{\prime}$ and $Z^{\prime}$ bosons. The current Tevatron limit is $720 \mathrm{GeV}$ for $W^{\prime}$ 82].

ATLAS and CMS have studied the sensitivity to a new neutral $Z^{\prime}$ boson in $e^{+} e^{-}, \mu \mu$ and jet-jet final states, for various masses and couplings [83, 84]. It is assumed that $\Gamma_{Z^{\prime}} \propto m_{Z^{\prime}}$. ATLAS finds the best sensitivity in the $e^{+} e^{-}$mode, in which signals could be seen up to $m_{Z^{\prime}}=5 \mathrm{TeV}$ for Standard-Model couplings. The other final states would provide important information on the $Z^{\prime}$ couplings. The pseudorapidity coverage over which lepton identification and measurement can be carried out is important for $Z^{\prime}$ searches: should a signal be observed, the forward-backward asymmetry of the charged leptons would provide important information on its nature. ATLAS found that reducing the lepton coverage from $|\eta| \leq 2.5$ to $|\eta| \leq 1.2$ roughly halved the observed asymmetries and prevented discrimination between two particular $Z^{\prime}$ models which they investigated.

ATLAS also investigated their sensitivity to a new charged boson $W^{\prime}$ decaying into $e \nu$. The signal is structure in the transverse mass distribution at masses much greater than $m_{W}$. Fig. 41 shows the signal for a $4 \mathrm{TeV} W^{\prime}$. They conclude that

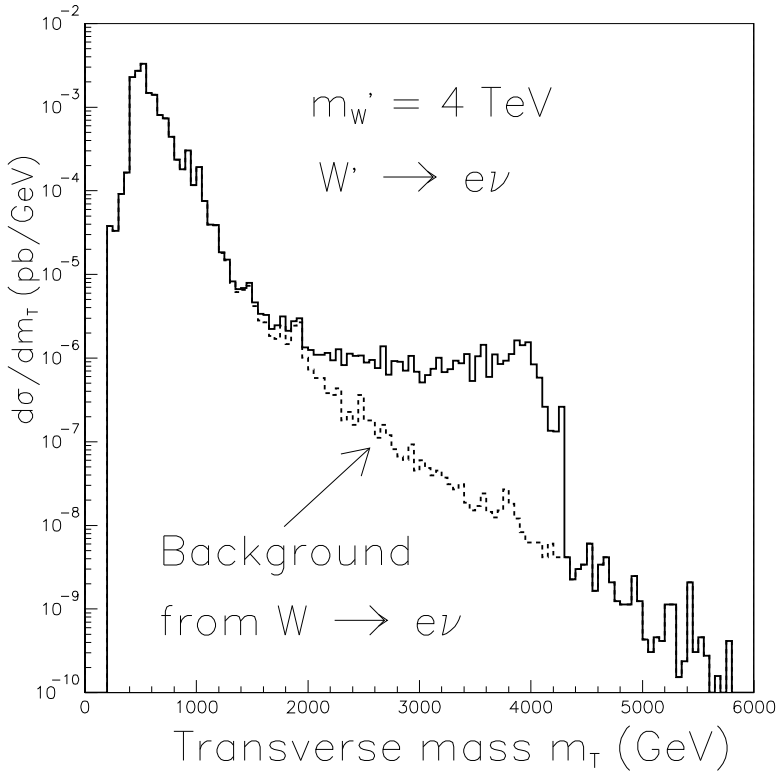

Figure 41: Expected electron-neutrino transverse mass distribution in ATLAS for $W^{\prime} \rightarrow e \nu$ decays with $M_{W^{\prime}}=4 \mathrm{TeV}$ (solid) above the dominant background from $W \rightarrow e \nu$ decays (dashed). From Ref. [1].

with $100 \mathrm{fb}^{-1}$ one would be sensitive to $m_{W^{\prime}}=6 \mathrm{TeV}$ and that the mass could be measured with a precision of $50 \mathrm{GeV}$. Similar results for the sensitivity to new $W^{\prime}$ bosons have also been obtained by CMS [85].

\section{EXTRA DIMENSIONS}

There is much recent theoretical interest in models of particle physics that have extra-dimensions in addition to the $3+1$ dimensions of normal space-time 24, 86, 25. In these models, new physics can appear at a mass scale of order $1 \mathrm{TeV}$ and can therefore be accessible at LHC. Two generic types of signals have been discussed. In models of large extra-dimensions [24], there is a tower of states consisting of massive graviton excitations whose properties are parameterized in terms of two parameters, the number $\delta$ of additional dimensions and the fundamental scale $M_{D}$. The size of the extra dimensions $R$ can be expressed in terms of these. Graviton excitations are produced in quark or gluon scattering; since they have gravitational strength couplings, they escape the detector, giving rise to final states with jets or photons plus missing transverse energy. Backgrounds arise from the production of $Z$ or $W$ in association with a jet [87]. Fig. 42 shows the distribution in missing transverse energy for the signal and background. The signal is manifest as an excess at large $\mathbb{E}_{T}$. For $\delta=2$, and an integrated luminosity of $100 \mathrm{fb}^{-1}$ values of $M_{D}$ less than $9 \mathrm{TeV}$ are accessible. The signal could be confirmed from the $\gamma+\mathbb{E}_{T}$ channel as the rates in this channel are predicted in terms of the same parameters.

In models of small (warped) extra dimensions [25], the graviton excitations are much more massive and decay into jets, lep- 


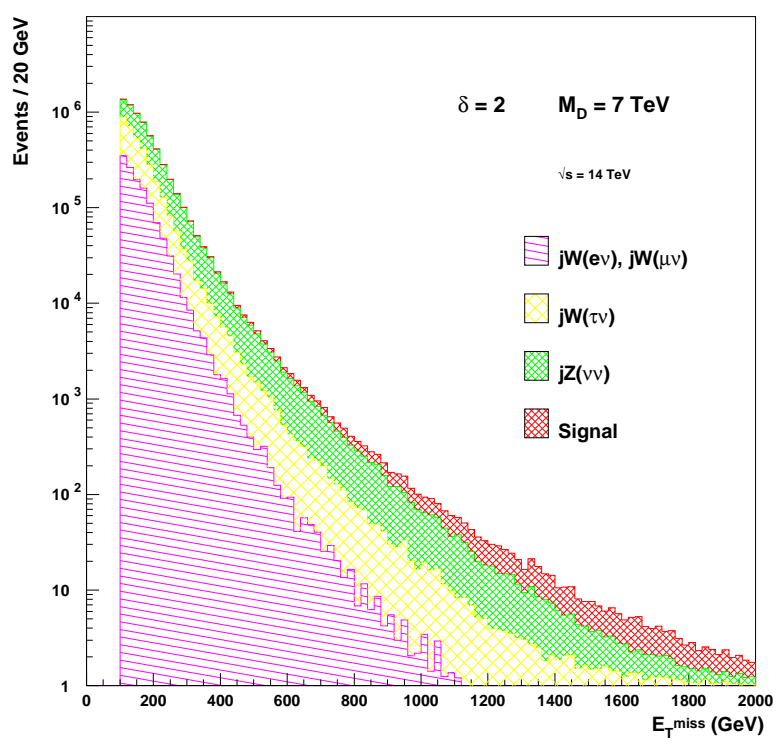

Figure 42: Distributions of the missing transverse energy in extra dimensions signal and in background events after the selection and for $100 \mathrm{fb}^{-1}$ of integrated luminosity. $\delta=2, M_{D}=7$ $\mathrm{TeV}$ is shown for the signal. From Ref. [87].

tons or photons. The decay into leptonic final states has been studied [88]. Signals are similar to those of new gauge bosons except that that graviton resonances have spin-2. Fig. 43 shows how such a resonance would appear in the $e^{+} e^{-}$mass distribution. The signal is visible for gravitons where $\sigma \times B \gtrsim 0.5 \mathrm{fb}$ or approximately $2 \mathrm{TeV}$ in the model used in Ref. [88]. Confirmation that the signal is indeed a graviton comes from measurements of the angular distribution that confirms that the resonance is spin- 2 and possible observation in other final states such as $\gamma \gamma$.

\section{ANOMALOUS GAUGE-BOSON COUPLINGS}

The trilinear $W W V$ and $Z \gamma V$ couplings $(V=Z, \gamma)$ may be probed at hadron colliders using diboson final states. Following the usual notation, the $\mathrm{CP}$-conserving $W W V$ anomalous couplings are parameterized by five parameters: $\Delta \kappa_{Z}, \lambda_{Z}, \Delta \kappa_{\gamma}$, $\lambda_{\gamma}$ and $\Delta g_{1}^{Z}$ [89]. In the Standard Model, $\kappa_{Z, \gamma}=1, \Delta g_{1}^{Z}=1$ and $\lambda_{Z, \gamma}=0$ In general, we would expect anomalous couplings of order $m_{W}^{2} / \Lambda^{2}$ if $\Lambda$ is the scale for new physics, so if $\Lambda \sim 1 \mathrm{TeV}$ then $\Delta \kappa_{V}, \lambda_{V} \sim 0.01$.

To maintain unitarity, anomalous couplings must be modified by a form factor; so (for example)

$$
\Delta \kappa_{V}\left(q^{2}\right)=\frac{\Delta \kappa_{V}^{0}}{\left(1+q^{2} / \Lambda_{F F}^{2}\right)^{n}}
$$

where $\Lambda_{F F}$ is the form factor scale and $n=2$ for $\Delta \kappa, \lambda$.

The ATLAS collaboration has studied [90] the sensitivity to anomalous couplings in the $W \gamma$ and $W Z$ modes; the $W^{+} W^{-}$

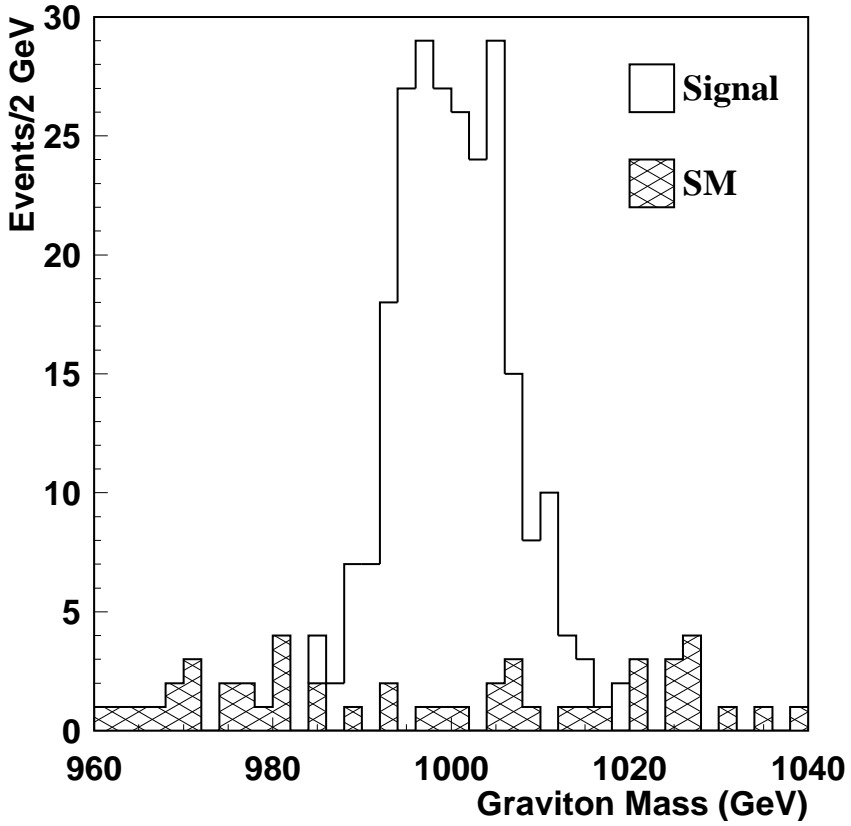

Figure 43: Distributions of the $e^{+} e^{-}$invariant mass in signal from a graviton resonance of mass $1 \mathrm{TeV}$ and in background after event selection and for $100 \mathrm{fb}^{-1}$ of integrated luminosity. From Ref. [88.

signal is swamped by $t \bar{t}$ background. A form factor scale $\Lambda_{F F}=10 \mathrm{TeV}$ was used. For the $W \gamma$ final state, events were assumed to be triggered using a high- $p_{T}$ lepton plus a high- $p_{T}$ photon candidate. The background includes contributions from events with a real lepton and a real photon (e.g. $b \bar{b} \gamma, t \bar{t} \gamma$, and $Z \gamma$ ); a fake lepton but a real photon (e.g. $\gamma+$ jet); and a fake photon with a real lepton (e.g. $W+$ jet, $b \bar{b}$, and $t \bar{t}$ ). Rejection factors of $10^{4}$ against jets faking photons and $10^{5}$ against jets faking electrons were used (consistent with the results from full simulation). To reduce backgrounds, events were selected with $p_{T}^{\gamma}>100 \mathrm{GeV}, p_{T}^{\ell}>40 \mathrm{GeV}$, and $\left|\eta^{\ell}\right|<2.5$. Events with jets were also vetoed, to further reduce backgrounds and to lessen the importance of higher-order QCD corrections. In an integrated luminosity of $100 \mathrm{fb}^{-1}, 7500$ events remain, with a signal to background ratio of $3: 1$. The $p_{T}^{\gamma}$ distribution is then fitted in the region where the Standard Model prediction is 15 events (above about $600 \mathrm{GeV}$ ), yielding limits of $\left|\Delta \kappa_{\gamma}\right|<0.04$ and $\left|\lambda_{\gamma}\right|<0.0025$ (95\% C.L.).

Similar techniques were used for the $W Z$ state. The trigger was three high- $p_{T}$ leptons, and the backgrounds are from $Z b \bar{b}, Z+$ jet, $b \bar{b}$ and $t \bar{t}$ processes. Events were selected with $p_{T}^{\ell}>25 \mathrm{GeV},\left|\eta^{\ell}\right|<2.5,\left|m_{\ell_{1} \ell_{2}}-m_{Z}\right|<10 \mathrm{GeV}^{2}$, and $m_{T}\left(\ell^{3}, \mathbb{E}_{T}\right)>40 \mathrm{GeV}^{2}$; a jet veto was also imposed. In $100 \mathrm{fb}^{-1}, 4000$ events then remain, with a signal to background ratio of $2: 1$. The $p_{T}^{Z}$ distribution is again fitted in the region where the Standard Model prediction is 15 events (above about $380 \mathrm{GeV})$, yielding limits of $\left|\Delta \kappa_{Z}\right|<0.07$ and $\left|\lambda_{Z}\right|<0.005$ (95\% C.L.).

A likelihood fit to the distributions then yields correlated limits on $\Delta \kappa_{Z}, \lambda_{Z}, \Delta g_{1}^{Z}, \Delta \kappa_{\gamma}$ and $\lambda_{\gamma}$ which are shown in 

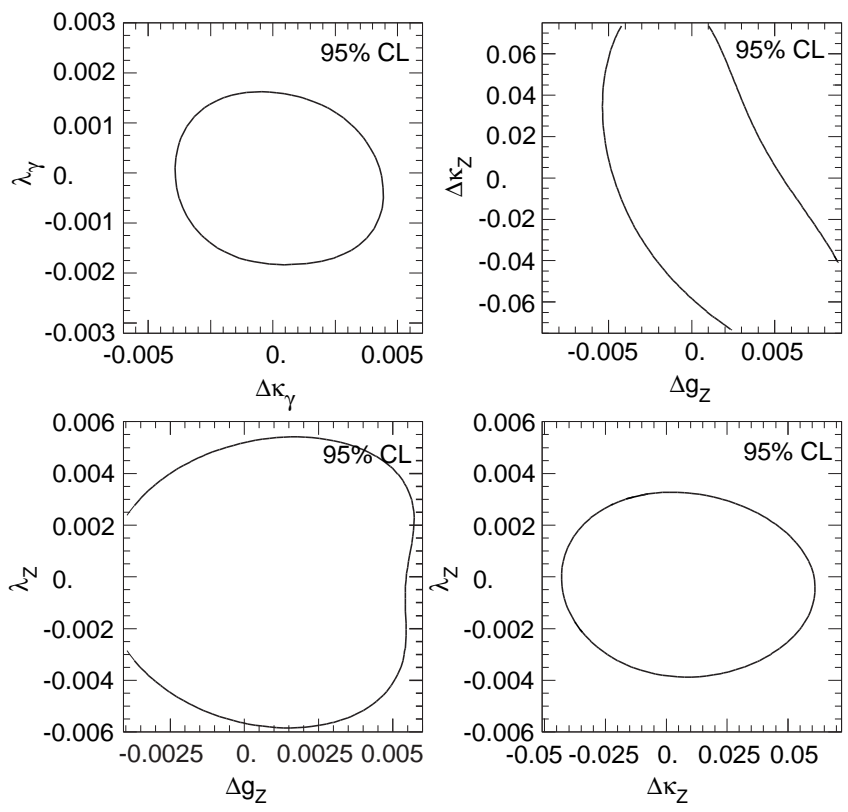

Figure 44: 95\% CL sensitivity limits on anomalous couplings from $W \gamma$ and $Z \gamma$ production for an integrated luminosity of $100 \mathrm{fb}^{-1}$. From Ref. [90].

Fig 44. These limits are comparable to deviations expected from radiative corrections in the Standard Model and extensions thereof [91]. Better precision might be obtained by using the angular distributions.

\section{STANDARD MODEL PHYSICS}

\section{A. Top Quark Physics}

The potential for the study of the top quark at hadron colliders is already apparent. The LHC will be a top factory, with about $10^{7} t \bar{t}$ pairs produced per year at a luminosity of $10^{33} \mathrm{~cm}^{-2} \mathrm{~s}^{-1}$. This would result in about 200,000 reconstructed $t \bar{t} \rightarrow(\ell \nu b)(j j b)$ events and 20,000 clean $e \mu$ events.

\section{Top Mass Measurement}

The top mass can be reconstructed from the $t \bar{t} \rightarrow(\ell \nu b)(j j b)$ final state using the invariant mass of the 3 -jet system. Problems arise from systematic effects due to the detector and the theoretical modeling of the production dynamics. This measurement requires, of course, that the hadronic calorimetry be calibrated to this level in the absolute energy scale and that its response be stable over time. ATLAS [35] has studied these effects and concludes that an accuracy of better than $\pm 2 \mathrm{GeV}$ could be attained. A complementary method exploits very high- $p_{T}$ top quarks, where the decay products are boosted and thus close. Combinatorics and uncertainties associated with measuring the individual jets are reduced, whereas those from jet energy calibrations are increased with the result that the expected errors are comparable.

The mass may also be reconstructed from dilepton events. ATLAS estimates that, by selecting events with two leptons from $W$ decays and an additional lepton from $b$-decay, and plotting the invariant mass of the lepton pair originating from the same top decay, the mass could be determined with a statistical accuracy of $\pm 0.5 \mathrm{GeV}$, and a total accuracy of about $\pm 2 \mathrm{GeV}$. The dominant systematic errors arise from uncertainties in the $b$-quark fragmentation and are therefore complementary to the 3 -jet system which is dominated by calorimeter and jet systematics.

\section{2. $\quad$ Rare Top Decays}

The large statistics available at LHC will provide sensitivity to other non-standard or rare top decays. As an example, ATLAS have investigated the channel $t \rightarrow Z c$ [35], which should occur at a negligible level in the SM. With an integrated luminosity of $100 \mathrm{fb}^{-1}$, branching ratios as small as $5 \times 10^{-5}$ could be measured.

It has been estimated that $\mathrm{LHC}$ will attain a precision 2-3 times better than that ultimately achievable at the Tevatron on the ratio of longitudinal to left-handed $W$ 's produced in $t$ decays. This ratio is exactly predicted in the SM for a given top mass, and is sensitive to non-standard couplings at the $t \rightarrow W b$ vertex, such as a possible $V+A$ contribution.

\section{B. B Physics}

The preceding sections have shown the importance of $b$ tagging in addressing many of the high- $p_{T}$ physics goals of the LHC. Both major detectors will consequently have the capability to tag heavy flavor production through displaced vertices. This capability, together with the large $b$-quark production cross-section at the LHC, will enable them to also pursue an interesting program of $B$-physics. It can be assumed that $\mathrm{CP}$ violation in the $b$-quark system will have been observed before the LHC gives data. Nevertheless the enormous rate will enable a very precise determination of $\sin 2 \beta$ to be made using the decay $B_{d} \rightarrow \Psi K_{S}(\Psi \equiv J / \psi, \psi(2 S))$. An error of \pm 0.02 can be expected after $10 \mathrm{fb}^{-1}$ of integrated luminosity. It will also be possible to measure $B_{s} \bar{B}_{s}$ mixing and to search for rare decays such as $B \rightarrow \mu \mu$.

\section{SUMMARY AND CONCLUSIONS}

The $S U(3) \times S U(2) \times U(1)$ gauge interactions of the Standard Model provide an elegant and a tremendously successful description of existing data, but they give no explanation of the origin of particle masses. The internal consistency of the Standard Model requires that at least part of the explanation of masses, the origin of electroweak symmetry breaking, must be found at the TeV scale. The LHC is unique among accelerators currently existing or under construction in that it has sufficient energy and luminosity to study that mass scale in detail. More specifically, the very detailed simulation studies carried out by the ATLAS and CMS collaborations enable one to make the following statements with a high degree of confidence:

- If the minimal Standard Model is correct and the Higgs boson is not discovered previously, it will be found at LHC. 
- If supersymmetry is relevant to the breaking of electroweak symmetry, it will be discovered at LHC and many details of the particular supersymmetric model will be disentangled.

- If the Higgs sector is that of the minimal supersymmetric model, at least one Higgs decay channel will be seen, no matter what the parameters turn out to be. In many cases, several Higgs bosons or decay channels will be seen.

- If the electroweak symmetry breaking proceeds via some new strong interactions, many resonances and new exotic particles will almost certainly be observed.

- New gauge bosons with masses less than several TeV will be discovered or ruled out.

- Signals for extra-dimensions will be revealed if the relevant scale is in the $\mathrm{TeV}$ range.

The LHC represents a great opportunity - and promise of vast excitement - not only for the collaborators on the LHC experiments but for the whole field of particle physics.

We thank our many ATLAS and CMS colleagues who have carried out the work summarized here.

The work was supported in part by the Director, Office of Energy Research, Office of High Energy Physics, Division of High Energy Physics of the U.S. Department of Energy under Contracts DE-AC03-76SF00098 and DE-AC02-98CH10886. Accordingly, the U.S. Government retains a nonexclusive, royaltyfree license to publish or reproduce the published form of this contribution, or allow others to do so, for U.S. Government purposes.

\section{REFERENCES}

[1] Atlas Technical proposal, CERN/LHCC/94-43.

[2] CMS Technical proposal, CERN/LHCC/94-38.

[3] M. Kobayashi, T. Maskawa, Prog. Theor. Phys. 49:652 (1973). N. Cabibbo, Phys. Rev. Lett. 10, 531 (1963).

[4] S. Glashow, Nucl. Phys. 22, 579 (1961); S. Weinberg, Phys. Rev. Lett. 19, 1264 (1967); A. Salam, in: "Elementary Particle Theory," W. Svartholm, ed., Almquist and Wiksell, Stockholm (1968); H.D. Politzer, Phys. Rev. Lett. 30, 1346 (73)); D.J. Gross and F.E. Wilczek, Phys. Rev. Lett. 30, 1343 (1973)).

[5] G. Miller, et al., Phys. Rev. D5, 6528 (1972), A. Bodek, et al., Phys. Rev. Lett. 30, 1087 (1973).

[6] J.J. Aubert, et al., Phys. Rev. Lett. 33, 1404 (1974); J.E. Augustin, et al., Phys. Rev. Lett. 33, 1406 (1974); G. Goldhaber, et al., Phys. Rev. Lett. 37, 255 (1976); S.W. Herb, et al., Phys. Rev. Lett. 39, 252 (1977); D. Andrews, et al., Phys. Rev. Lett. 45, 219 (1980).

[7] F.J. Hasert, et al., Phys. Lett. 46B, 138 (1973).

[8] R. Brandelik, et al., Phys. Lett. 86B, 243 (1979); D.P. Barber, et al., Phys. Rev. Lett. 43, 830 (1979); C. Berger, et al., Phys. Lett. 86B, 418 (1979);W. Bartel, et al., Phys. Lett. 91B, 142 (1980), P. Banner et al., Phys. Lett. 122B, 130 (1983), Phys. Lett. 129B, 476 (1983).
[9] G. Arnison, et al. Phys. Lett. 126B, 398 (1983), G. Arnison, et al. Phys. Lett. 122B, 103 (1983).

[10] F. Abe et al. Phys. Rev. Lett. 73, 2667 (1994), Phys. Rev. D52, 2605 (1995); S. Abachi et al. Phys. Rev. Lett. 74, 2632 (1995).

[11] K. Kodama et al. [DONUT Collaboration], hep-ex/0012035.

[12] H. Christenson, J. W. Cronin, V. L. Fitch and R. Turlay, Phys. Rev. Lett. 13 (1964) 138.

[13] A. Alavi-Harati et al. Phys. Rev. Lett. 83, 22 (1999); T. Gershon hep-ex/0101034.

[14] D. Boutigny, et al., SLAC-R-95-457, M.T. Cheng, et al., KEK Report BELLE-TDR-3-95.

[15] BaBar collaboration, hep-ex/0102030; Belle Collaboration hepex/0102018.

[16] T. Affolder et al., Phys. Rev. D61, 072005 (2000).

[17] D. E. Groom et al. [Particle Data Group Collaboration], Eur. Phys. J. C 15, 1 (2000).

[18] R.N. Cahn, R. N., LBL-38649 (1996), submitted to Rev. Mod. Phys.

[19] P. Igo-Kemenes, report to the LEPC, November 2000,

[20] The LEP Electroweak Working Group, CERN-EP-2000-016.

[21] C. Quigg, B.W. Lee and H. Thacker, Phys. Rev. D16, 1519 (77) M. Veltman, Acta Phys. Polon. B8:475 (1977).

[22] For a review see, I. Hinchliffe, Ann. Rev. Nucl. and Part. Sci. 36, 505 (1986);

[23] For a review see, K.D. Lane hep-9605257 (1996).

[24] N. Arkani-Hamed, S. Dimopoulos and G. Dvali, Phys. Lett. B429, 263 (1998).

[25] L. Randall and R. Sundrum, Phys. Rev. Lett. 83, 3370 (1999).

[26] P. Lefevre, et al., CERN/AC/95-05.

[27] ALICE Technical Proposal, CERN/LHCC/95-71.

[28] LHC-B Technical Proposal, CERN/LHCC/95-XX.

[29] E. Eichten, et al., Rev. Mod. Phys. 56, 579 (84).

[30] CERN Program Library, GEANT.

[31] C. Seez, CMS-TN/94-289 (1994).

[32] D. Froidevaux. F. Gianotti and E. Richter Was, ATLAS Note PHYS-NO-064 (1995), F. Gianotti and I. Vichon, ATLAS Note PHYS-NO-078 (1996).

[33] F. Abe, et al., Phys. Rev. D51, 4623 (1995).

[34] N. Stepanov and V. Drollinger, CMS Note 2000/059.

[35] ATLAS Collaboration, ATLAS Detector and Physics Performance Technical Design Report, CERN/LHCC/99-14, http: //atlasinfo.cern.ch/Atlas/GROUPS /PHYSICS/TDR/access.html.

[36] V. Drollinger, PhD Thesis, University of Karlsruhe, 2001.

[37] J.-C. Chollet, et al., ATLAS internal note PHYS-NO-17 (1992), L. Poggioli, ATLAS Note PHYS-NO-066 (1995).

[38] D. Denegri, R. Kinnunen and G. Roullet, CMS-TN/93-101 (1993).

[39] V. Drollinger and A. Sopczak, hep-ph/0102342,

[40] CMS Collaboration, ECAL Project Technical Design Report, CERN/LHCC/97-33, http: //cmsdoc.cern.ch/cms/TDR/ECAL/ecal.html. 
[41] Ph. Mine, S. Moreau and I.Puljak, CMS Note 1999/071; I. Puljak PhD Thesis, Ecole Polytechnique, 2000.

[42] M. Dittmar and H. Dreiner, Phys. Rev. D 55, 167 (1997). [hep$\mathrm{ph} / 9608317]$.

[43] D. Bomestar et al., CMS TN-1995/018.

[44] M. Dittmar, hep-ex/9901009.

[45] M. Bosman and M. Nessi, ATLAS Note PHYS-NO-050 (1994).

[46] J. Ohnemus, Phys. Rev. D50, 1931 (1994).

[47] N.Stepanov, CMS-TN/93-87 (1993); S. Abdullin and N. Stepanov, CMS-TN/94-179 (1994).

[48] S. Abdullin and N. Stepanov, CMS-TN/94-178 (1994).

[49] D. Zeppenfeld, R. Kinnunen, A. Nikitenko and E. Richter-Was, Phys. Rev. D 62, 013009 (2000) [hep-ph/0002036].

[50] M. Carena, M. Quiros, C.E.M. Wagner, Nucl. Phys. B461, 407 (96).

[51] S. Abdullin, C. Kao and N. Stepanov, University of Rochester UR-1475, July 1996.

[52] R. Kinnunen, J. Tuominiemi, and D. Denegri, CMS-TN/93-98 (1993) and CMS-TN/93-103; C. Seez, CMS-TN/93-84.

[53] D. Cavalli, et al., ATLAS Note PHYS-NO-025 (1993).

[54] D. Cavalli and S. Resconi, ATL-PHYS-2000-005.

[55] R. Kinnunen and A. Nikitenko, CMS Note 2001/031.

[56] R. Kinnunen, CMS NOTE-2000/045.

[57] K.A. Assamagan and Y. Coadou, ATL-PHYS-2000-031.

[58] N. Stepanov, CMS-TN/94-182 (1994).

[59] D. Denegri et al, CMS Note 2001/032.

[60] L. Alvarez-Gaume, J. Polchinski and M.B. Wise, Nucl. Phys. B221, 495 (1983);

L. Ibañez, Phys. Lett. 118B, 73 (1982);

J.Ellis, D.V. Nanopolous and K. Tamvakis, Phys. Lett. 121B, 123 (1983);

K. Inoue et al. Prog. Theor. Phys. 68, 927 (1982);

A.H. Chamseddine, R. Arnowitt, and P. Nath, Phys. Rev. Lett., 49, 970 (1982).

[61] M. Dine, W. Fischler and M. Srednicki, Nucl. Phys. B189, 575 (1981);

S. Dimopoulos and S. Raby, Nucl. Phys. B192, 353 (1981);

C. Nappi and B. Ovrut, Phys. Lett.113B, 175 (1982);

L. Alvarez-Gaumé, M. Claudson and M. Wise, Nucl. Phys. B207, 96 (1982);

M. Dine and A. Nelson, Phys. Rev. D48, 1227 (1993);

M. Dine, A. Nelson and Y. Shirman, Phys. Rev. D51, 1362 (1995);

M. Dine, et al., Phys. Rev. D53, 2658 (1996).

[62] L. Randall and R. Sundrum, Nucl. Phys. B 557, 79 (1999); G. F. Giudice, M. A. Luty, H. Murayama and R. Rattazzi, JHEP9812, 027 (1998)

[63] S. Abdullin et al. [CMS Collaboration], "Discovery potential for supersymmetry in CMS," hep-ph/9806366.

[64] I. Hinchliffe and F. E. Paige, hep-ph/0010086, to appear in Phys. Rev. D.

[65] D. Denegri, W. Majerotto and L. Rurura, Phys. Rev. D60 (1999), 035008;

L. Rurua, PhD Thesis, Institute of High Energy Physics, Austrian Academy of Sciences, 1999.
[66] I. Hinchliffe, F. E. Paige, M. D. Shapiro, J. Sodergvist and W. Yao, Phys. Rev. D 55, 5520 (1997) [hep-ph/9610544].

[67] H. Bachacou, I. Hinchliffe and F. E. Paige, Phys. Rev. D 62, 015009 (2000) hep-ph/9907518.

[68] H. N. Brown et al. [Muon g-2 Collaboration], Phys. Rev. Lett. 86, 2227 (2001)

[69] B. C. Allanach, C. G. Lester, M. A. Parker and B. R. Webber, JHEP0009, 004 (2000) [hep-ph/0007009].

[70] S. Abdullin CMS Note 1997/070.

[71] M. Kazana, G. Wrochna, and P. Zalewski, CMS CR 1999/019 (June, 1999).

[72] S. Ambrosanio, B. Mele, A. Nisati, S. Petrarca, G. Polesello, A. Rimoldi and G. Salvini, hep-ph/0012192.

[73] F. E. Paige and J. Wells, hep-ph/0001249.

[74] B. C. Allanach, A. J. Barr, L. Drage, C. G. Lester, D. Morgan, M. A. Parker, P. Richardson and B. R. Webber, hep-ph/0102173.

[75] F. Moortgat, CMS CR 2001/005.

[76] M.S. Chanowitz and M.K. Gaillard, Nucl. Phys. B261, 379 (1985).

[77] J.R. Smith, CMS TN/95-179 (1995).

[78] S. Dimopoulos and L. Susskind, Nucl. Phys. B 155, 237 (1979).

[79] S. Weinberg, Phys. Rev. D 19, 1277 (1979).

[80] C. T. Hill, model," Phys. Lett. B 266, 419 (1991).

[81] R. Casalbouni, et al., Int. J. Mod. Phys.A4,1065 (1989).

[82] F. Abe, et al., Phys. Rev. Lett. 74, 2900 (95), Phys. Rev. D51, 949 (1995).

[83] A. Henriquez and L. Poggioli, ATLAS Note PHYS-NO-010 (1992).

[84] C. Wulz, CMS TN-1993/107.

[85] A. Caner and M. Spezziga, CMS Note 2000/072.

[86] I. Antoniadis, Phys. Lett. B246, 377 (1990).

[87] L. Vacavant and I. Hinchliffe, SN-ATLAS-2001-005, J. Phys. G (to appear).

[88] B. C. Allanach, K. Odagiri, M. A. Parker and B. R. Webber, JHEP0009, 019 (2000) [hep-ph/0006114].

[89] K. Hagiwara, et al. Nucl. Phys. B282, 253 (1987)

[90] G. Azuelos, et al., ATL-PHYS-2001-002.

[91] J. Ellison and J. Wudka, Ann. Rev. Nucl. and Part. Sci. 48, 33 (1998)

[92] D. Cavalli, et al., ATLAS internal note PHYS-NO-053 (1994).

[93] R. Kinnunen, D. Denegri and J. Tuominiemi, CMS-TN/94-233 (1994).

[94] 'Report of the TeV2000 Study Group on Future Electroweak Physics at the Tevatron,' D. Amidei and R. Brock (eds.), Fermilab 1996. 